\title{
Constraints on anomalous Higgs boson couplings to vector bosons and fermions in its production and decay using the four-lepton final state
}

\author{
A. M. Sirunyan et al. \\ (CMS Collaboration)
}

(Received 25 April 2021; accepted 6 July 2021; published 17 September 2021)

\begin{abstract}
Studies of $C P$ violation and anomalous couplings of the Higgs boson to vector bosons and fermions are presented. The data were acquired by the CMS experiment at the LHC and correspond to an integrated luminosity of $137 \mathrm{fb}^{-1}$ at a proton-proton collision energy of $13 \mathrm{TeV}$. The kinematic effects in the Higgs boson's four-lepton decay $H \rightarrow 4 \ell$ and its production in association with two jets, a vector boson, or top quarks are analyzed, using a full detector simulation and matrix element techniques to identify the production mechanisms and to increase sensitivity to the tensor structure of the Higgs boson interactions. A simultaneous measurement is performed of up to five Higgs boson couplings to electroweak vector bosons $(H V V)$, two couplings to gluons $(H g g)$, and two couplings to top quarks $(H t t)$. The $C P$ measurement in the $H t t$ interaction is combined with the recent measurement in the $H \rightarrow \gamma \gamma$ channel. The results are presented in the framework of anomalous couplings and are also interpreted in the framework of effective field theory, including the first study of $C P$ properties of the $\mathrm{Htt}$ and effective $\mathrm{Hgg}$ couplings from a simultaneous analysis of the gluon fusion and top-associated processes. The results are consistent with the standard model of particle physics.
\end{abstract}

DOI: $10.1103 /$ PhysRevD.104.052004

\section{INTRODUCTION}

The discovery by the ATLAS and CMS Collaborations of a Higgs boson $(H)$ with a mass of $\approx 125 \mathrm{GeV}[1-3]$ has confirmed the predictions of the standard model (SM) of particle physics [4-10]. The CMS [11-18] and ATLAS [19-25] experiments have set constraints on the spin-parity properties of the $H$ boson and anomalous $H V V$ couplings, where $V$ stands for $W, Z$, and $\gamma$ electroweak (EW) gauge bosons, finding its quantum numbers to be consistent with $J^{P C}=0^{++}$, but leaving room for small anomalous $H V V$ couplings. In theories beyond the SM (BSM), $H$ boson interactions may generate several of them, which lead to new interaction tensor structures, both $C P$ even and $C P$ odd. The new anomalous tensor structures of the $H$ boson interactions may also appear through loop corrections in SM processes, but the size of their contributions is beyond the current experimental sensitivity. The $C P$-odd anomalous couplings between the $H$ boson and either the top quark or new BSM particles, fermions or bosons, contributing to the gluon fusion loop may generate $C P$ violation in $H g g$ interactions (where $g$ stands for gluon). Possible $C P$

${ }^{*}$ Full author list given at the end of the article.

Published by the American Physical Society under the terms of the Creative Commons Attribution 4.0 International license. Further distribution of this work must maintain attribution to the author(s) and the published article's title, journal citation, and DOI. Funded by SCOAP . violation effects in couplings to fermions, $H \mathrm{ff}$, had not been experimentally probed until recently, when the first constraints were reported by CMS [26] and ATLAS [27] in $t \bar{t} H$ production (where $t$ stands for top quark) using the $H \rightarrow \gamma \gamma$ channel.

In this paper, we study the tensor structure of the $H V V$, $\mathrm{Hgg}$, and $\mathrm{Htt}$ interactions, and we search for several anomalous effects, including $C P$ violation, using the four-lepton final state $H \rightarrow V V \rightarrow 4 \ell$, where $\ell=\mu$ or $e$. The $H$ boson production processes considered in this paper include gluon fusion $(g g H)$, vector boson fusion (VBF), associated production with a weak vector boson $(\mathrm{VH}$, either $Z H$ or $W H)$, with a top quark pair $(t \bar{t} H)$, with a single top quark $(t H)$, and with a bottom quark pair $(b \bar{b} H)$. The Feynman diagrams for these processes are shown in Figs. 1-5 and discussed in detail in Sec. II. Kinematic effects in the $H$ boson's decay and its production in association with two jets generated in either the VBF or $g g H$ processes, with a vector boson, or with top quarks are analyzed. Production and decay processes of the $H$ boson are sensitive to certain anomalous contributions, or equivalently higher-dimensional operators in the effective field theory (EFT) [28], which modify the kinematic distributions of the $H$ boson's decay products and of the particles produced in association with the $H$ boson. Prior measurements of EW processes limit the allowed values of certain EFT operators, and the preferred EFT basis used in this paper is chosen to minimize the number of independent operators and their correlations. This allows us to reduce 
the number of operators to be measured. The results are also translated and reported in different, frequently used EFT bases. The effects of EFT modifications in backgrounds are found to be negligible because of the high purity of the signal peak in the four-lepton invariant mass distribution and further constraints on the backgrounds from sidebands.

Each production process of the $H$ boson is identified using its kinematic features, and events are assigned to corresponding categories. Two categorization schemes are employed in this analysis, one designed to study $\mathrm{Htt}$ and $H g g$ and the other designed to study $H V V$ anomalous couplings. Within each category, the matrix element likelihood approach (MELA) [29-33] is employed to construct observables that are optimal for the measurement of the chosen anomalous couplings, or EFT operators, including $C P$-sensitive observables to search for $C P$-violating operators. In our approach, fully simulated Monte Carlo (MC) signal samples that include anomalous couplings allow the incorporation of detector effects into the likelihood analysis, and observables explore all kinematic features of the events, including those sensitive to $C P$ violation and to simultaneous anomalous effects in the production and decay of the $H$ boson. These features distinguish our analysis from the recent measurements by the ATLAS Collaboration in the same $H \rightarrow 4 \ell$ decay channel [34,35], in which case the measurements are derived from the differential distributions based on either the simplified template cross sections [28] or unfolded fiducial measurements.

We follow the formalism used in the study of anomalous couplings in the earlier analyses of CMS data in Refs. $[11-18,26]$. We focus on the measurements where the $H$ boson is produced on shell; the extension to the off shell region is considered in Ref. [17], where joint constraints on the $H$ boson width $\Gamma_{H}$ and its anomalous couplings are obtained using a partial dataset. Some of the theoretical foundations relevant to the present analysis can be found in Refs. [28-33,36-66]. The results are presented in a model-independent way, which allows interpretation in the scattering amplitude or effective Lagrangian approach, for example in the frameworks of the standard model effective field theory (SMEFT) [67-70] or pseudo observables [28].

Compared to our previous results on anomalous $H V V$ couplings [16,17], which used a subset of the data presented here, several substantial improvements have been introduced. First, as a result of the increased number of $H \rightarrow 4 \ell$ events, the expected $95 \%$ confidence level (CL) constraints on $H V V$ couplings are now dominated by the tight limits from the analysis of kinematic distributions of particles produced in association with the $H$ boson in the VBF and $V H$ processes. Second, an improved fit implementation allows for the simultaneous measurement of up to five independent $H V V$, two $H g g$, and two $H t t$ couplings using the single decay channel $H \rightarrow 4 \ell$. The couplings are parametrized with the signal strength and the fractional cross section contributions of the anomalous couplings. A direct constraint on $C P$ violation in the $H g g$ coupling is obtained for the first time by employing $C P$-sensitive observables. The $\mathrm{CP}$ violation measurement in the $\mathrm{Htt}$ coupling closely follows our recent measurement in the $H \rightarrow \gamma \gamma$ channel [26], and the results are combined. We also perform the first study of $C P$ properties in the Htt and effective $H g g$ couplings from a simultaneous analysis of the gluon fusion and top-associated processes. For all anomalous couplings, we interpret our results via the SMEFT framework in terms of $\mathrm{HVV}, \mathrm{Hgg}$, and $\mathrm{Htt}$ operators.

The rest of this paper is organized as follows. The phenomenology of anomalous $H V V, H g g$, and $H$ ff couplings and considerations in the EFT framework are discussed in Sec. II. The data and MC simulation, event reconstruction, and selection are discussed in Sec. III. The kinematic variables associated with the $H$ boson's production and decay and its MELA analysis are introduced in Sec. IV. The implementation of the maximum likelihood fit is shown in Sec. V. The results are presented and discussed in Sec. VI. We conclude in Sec. VII.

\section{PARAMETRIZATION OF $H$ BOSON PRODUCTION AND DECAY PROCESSES}

The goal of this study is to search for $C P$ violation and, more generally, anomalous couplings of the $H$ boson, in its interactions with fermions and vector bosons in the production and decay processes. These potential sources of $C P$ violation and anomalous tensor structures of interactions may arise from BSM effects, including those considered in the EFT formulation. The dominant processes sensitive to such interactions are shown in Figs. 1-5 [28].

The main decay process considered in this paper is $H \rightarrow V V \rightarrow 4 \ell$, with the $H V V$ vertex shown in Fig. 1, right. The dominant $H$ boson production mechanism is gluon fusion $g g H$, shown in Fig. 1, left. The dominant contribution to the gluon fusion loop comes from the top quark, with smaller contributions from the bottom quark and lighter quarks. However, contribution of new BSM states to the loop and variation of $C P$ properties of the $H$ boson couplings to SM quarks are also possible and are considered in this paper. The $H V V$ vertex also appears in the vector boson fusion $\mathrm{VBF}$ and associated production with a weak vector boson $Z H$ or $W H$, shown in Fig. 2, which are the next dominant production mechanisms of the $H$ boson.

The production of an $H$ boson in association with a top quark pair $t \bar{t} H$ is shown in Fig. 3, left. This is the main channel that allows to study the $C P$ property in the $H$ boson coupling to fermions. We also combine our results with the recent $t \bar{t} H$ measurements in the $H \rightarrow \gamma \gamma$ channel [26]. The production of an $H$ boson in association with a single top quark $t H$ is shown in Fig. 4. This production receives 


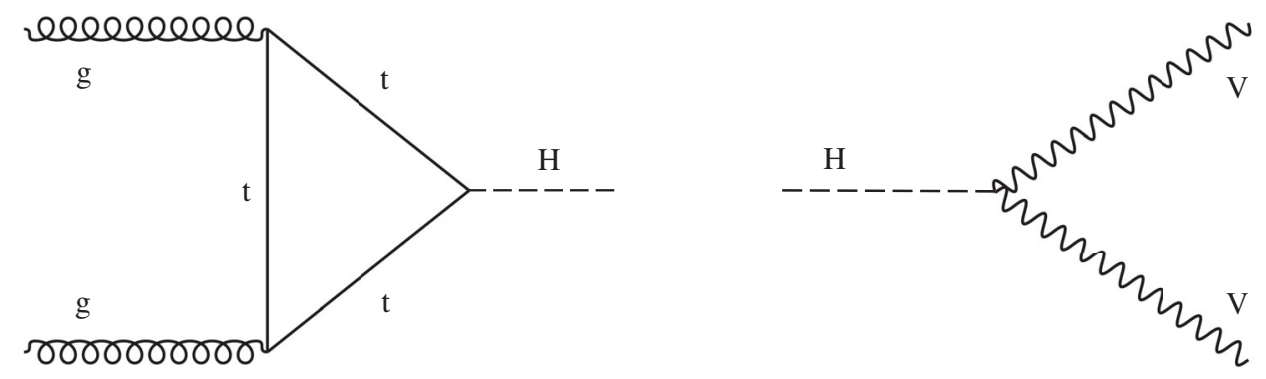

FIG. 1. Leading-order Feynman diagrams for the gluon fusion production mode (left) and $H \rightarrow V V$ decay (right).
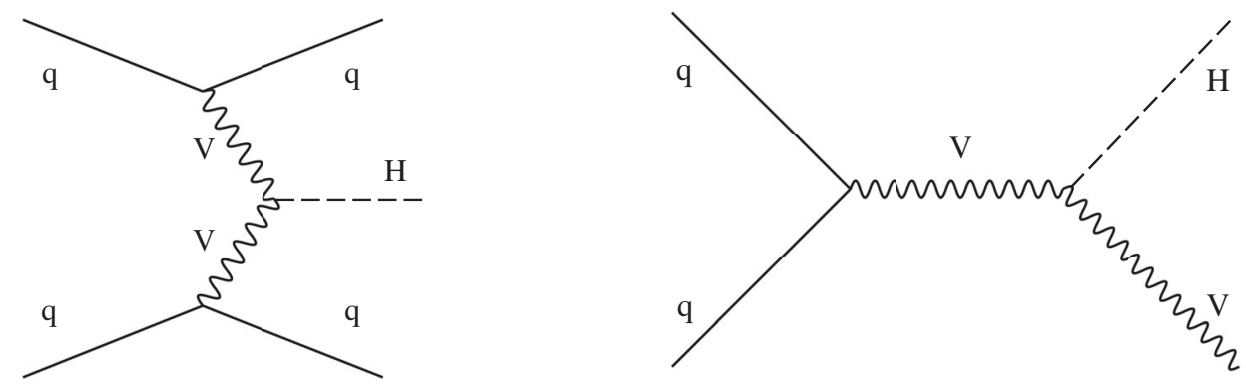

FIG. 2. Leading-order Feynman diagrams for the VBF (left) and $V H$ (right) production modes.
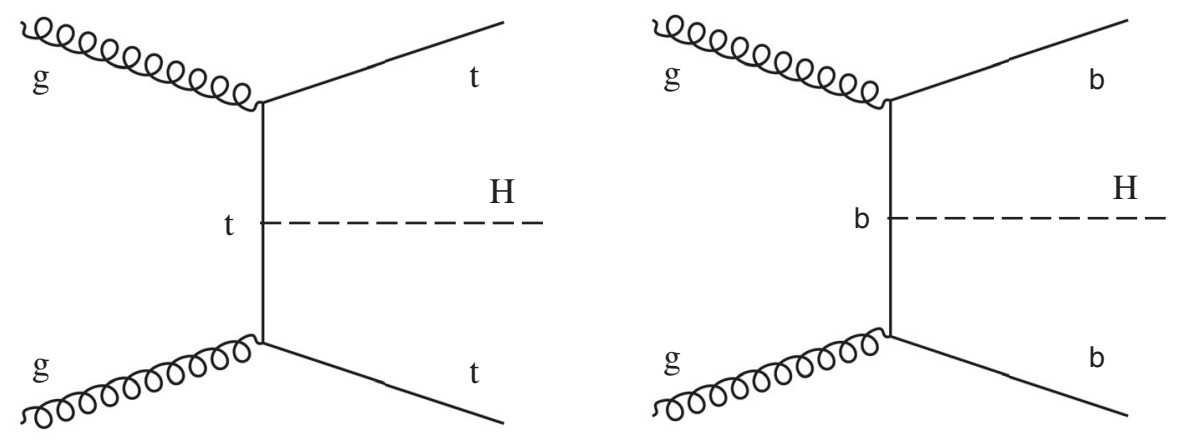

FIG. 3. Examples of leading-order Feynman diagrams for the $t \bar{t} H$ (left) and $b \bar{b} H$ (right) production modes.

contributions from both $H V V$ and $H t t$ couplings, but its expected cross section is smaller than that of $t \bar{t} H$ production. Both $H V V$ and $H t t$ couplings also contribute to the $g g \rightarrow Z H$ production mode, shown in Fig. 5. However, this gluon fusion production mode of $Z H$ is expected to contribute only about $5 \%$ of the $V H$ process cross section shown in Fig. 2, the dependence on anomalous $H V V$ couplings is suppressed in this process [33], and for these reasons this production mode is neglected in this analysis. Finally, we also consider the $b \bar{b} H$ production mode shown in Fig. 3, right. However, this process does not provide kinematic features that could distinguish the $C P$ structure
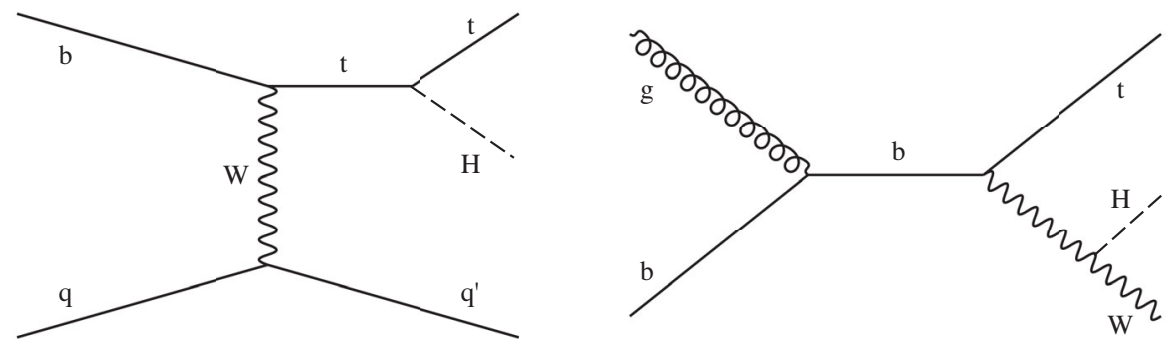

FIG. 4. Examples of leading-order Feynman diagrams for the $t H$ production mode. 

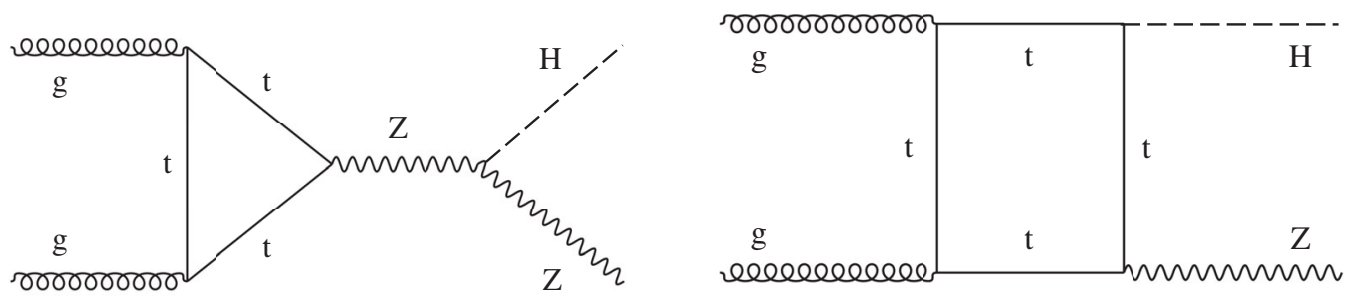

FIG. 5. Leading-order Feynman diagrams for the $g g \rightarrow Z H$ production mode.

of interactions [32] or the experimental signatures that would allow its isolation from the other more dominant production mechanisms.

\section{A. Parametrization of production and decay amplitudes}

Anomalous effects in the $H$ boson couplings to fermions, such as in the $t \bar{t} H$ and $b \bar{b} H$ production and partially in the $t H$ and $g g \rightarrow Z H$ production, can be parametrized with the amplitude

$$
A(H \mathrm{ff})=-\frac{m_{\mathrm{f}}}{v} \bar{\psi}_{\mathrm{f}}\left(\kappa_{\mathrm{f}}+\mathrm{i} \tilde{\kappa}_{\mathrm{f}} \gamma_{5}\right) \psi_{\mathrm{f}},
$$

defined for each fermion type $\mathrm{f}$, where $\bar{\psi}_{\mathrm{f}}$ and $\psi_{\mathrm{f}}$ are the fermions' Dirac spinors, $\kappa_{\mathrm{f}}$ and $\tilde{\kappa}_{\mathrm{f}}$ are the corresponding coupling strengths, $m_{\mathrm{f}}$ is the fermion mass, and $v$ is the SM Higgs field vacuum expectation value. In the SM, the coupling strengths are $\kappa_{\mathrm{f}}=1$ and $\tilde{\kappa}_{\mathrm{f}}=0$. The presence of both $C P$-even $\kappa_{\mathrm{f}}$ and $C P$-odd $\tilde{\kappa}_{\mathrm{f}}$ couplings will lead to $C P$ violation. In an experimental analysis of the $b \bar{b} H$ process it is not possible to resolve the $\kappa_{b}$ and $\tilde{\kappa}_{b}$ couplings [32], but it is possible to resolve the $\kappa_{t}$ and $\tilde{\kappa}_{t}$ couplings in the $t \bar{t} H$ and $t H$ processes, which we explore in this paper.

Anomalous effects in EW $H$ boson production (VBF, $Z H$, and $W H), g g H$ production, $H \rightarrow V V$ decay, and partially in the $t H$ and $g g \rightarrow Z H$ production, are described by the $H V_{1} V_{2}$ couplings. The scattering amplitude describing the interaction between a spin-zero $H$ boson and two spin-one gauge bosons $V_{1} V_{2}$, such as $Z Z, Z \gamma, \gamma \gamma, W W$, or $g g$, is written as

$A\left(H V_{1} V_{2}\right)=\frac{1}{v}\left[a_{1}^{V V}+\frac{\kappa_{1}^{V V} q_{V 1}^{2}+\kappa_{2}^{V V} q_{V 2}^{2}}{\left(\Lambda_{1}^{V V}\right)^{2}}+\frac{\kappa_{3}^{V V}\left(q_{V 1}+q_{V 2}\right)^{2}}{\left(\Lambda_{Q}^{V V}\right)^{2}}\right] m_{V 1}^{2} \epsilon_{V 1}^{*} \epsilon_{V 2}^{*}+\frac{1}{v} a_{2}^{V V} f_{\mu \nu}^{*(1)} f^{*(2), \mu \nu}+\frac{1}{v} a_{3}^{V V} f_{\mu \nu}^{*(1)} \tilde{f}^{*(2), \mu \nu}$,

where $f^{(i) \mu \nu}=\epsilon_{V i}^{\mu} q_{V i}^{\nu}-\epsilon_{V i}^{\nu} q_{V i}^{\mu}, \quad \tilde{f}_{\mu \nu}^{(i)}=\frac{1}{2} \epsilon_{\mu \nu \rho \sigma} f^{(i), \rho \sigma}$, and $\epsilon_{V i}, q_{V i}$, and $m_{V i}$ are the polarization vector, fourmomentum, and pole mass of a gauge boson $i=1$ or 2 . The constants $\Lambda_{1}$ and $\Lambda_{Q}$ are the scales of BSM physics necessary to keep the $\kappa_{i}^{V V}$ couplings unitless, and $a_{1}^{V V}, a_{2}^{V V}$, $a_{3}^{V V}, \kappa_{1}^{V V}, \kappa_{2}^{V V}$, and $\kappa_{3}^{V V}$ are real numbers that modify the corresponding amplitude terms. Equation (2) describes couplings to both EW bosons and gluons, so $H V_{1} V_{2}$ can stand for $H V V$ or $\mathrm{Hgg}$.

In Eq. (2), the only nonzero tree-level contributions in the $\mathrm{SM}$ are $a_{1}^{Z Z} \neq 0$ and $a_{1}^{W W} \neq 0$. In the SM, $a_{1}^{Z Z}=a_{1}^{W W}=2$. The rest of the $Z Z$ and $W W$ couplings are considered to be anomalous contributions, which are either small contributions arising in the SM because of loop effects or new BSM contributions. Among the anomalous contributions, considerations of symmetry and gauge invariance require $\kappa_{1}^{Z Z}=\kappa_{2}^{Z Z}, \kappa_{1}^{W W}=\kappa_{2}^{W W}$, and $\quad a_{1}^{Z \gamma}=a_{1}^{\gamma \gamma}=a_{1}^{g g}=\kappa_{1}^{\gamma \gamma}=\kappa_{2}^{\gamma \gamma}=\kappa_{1}^{g g}=\kappa_{2}^{g g}=\kappa_{1}^{Z \gamma}=\kappa_{3}^{V V}=0$ [33]. Therefore, there are a total of 13 independent parameters describing couplings of the $H$ boson to EW gauge bosons and two parameters describing couplings to gluons. The presence of any of the $C P$-odd couplings $a_{3}^{V V}$ together with any of the other couplings, which are all $C P$ even, will lead to $C P$ violation in a given process.

Since in our analysis it is not possible to disentangle the top quark, bottom quark, and any other heavy BSM particle contributions to the gluon fusion loop from kinematic features of the event, we parametrize the $H g g$ coupling with only two parameters: $C P$-even $a_{2}^{g g}$ and $C P$-odd $a_{3}^{g g}$, which absorb all SM and BSM loop contributions. However, when the gluon fusion process is analyzed jointly with the $t \bar{t} H$ and $t H$ processes, it may be possible to disentangle the top quark contributions in the loop from the relative rates of the processes, and we allow these contributions to be separated.

\section{B. Symmetry considerations and SMEFT formulation}

The formulation in Eqs. (1) and (2) is presented in the approach of anomalous amplitude decomposition. However, it is fully equivalent to the Lagrangian parametrization with dimension-4 operators, such as the $a_{1}^{V V}$ term in Eq. (2), and dimension-6 operators, such as the other terms in Eq. (2), using the mass eigenstate basis [28]. The dimension- 8 and higher-dimension contributions are 
neglected. We apply additional symmetry considerations discussed below, which reduce the number of independent parameters to be measured. The chosen basis coincides with the Higgs basis [28] under $\mathrm{SU}(2) \times \mathrm{U}(1)$ symmetry. The choice of the EFT operator basis is motivated by the natural parametrization in terms of observable states and, as a consequence, allows a more transparent construction of the data analysis and presentation of the results. However, we also present results in the Warsaw basis [28], using tools in Refs. [33,71] to perform the translation. Our approach with $\mathrm{SU}(2) \times \mathrm{U}(1)$ symmetry is equivalent to the SMEFT formulation [28].

The parametrization in Eq. (2) is the most general one, and we apply $\mathrm{SU}(2) \times \mathrm{U}(1)$ symmetry in the relationships of anomalous couplings as follows $[28,33]$ :

$$
\begin{gathered}
a_{1}^{W W}=a_{1}^{Z Z}+\frac{\Delta m_{W}}{m_{W}}, \\
a_{2}^{W W}=c_{\mathrm{w}}^{2} a_{2}^{Z Z}+s_{\mathrm{w}}^{2} a_{2}^{\gamma \gamma}+2 s_{\mathrm{w}} c_{\mathrm{w}} a_{2}^{Z \gamma}, \\
a_{3}^{W W}=c_{\mathrm{w}}^{2} a_{3}^{Z Z}+s_{\mathrm{w}}^{2} a_{3}^{\gamma \gamma}+2 s_{\mathrm{w}} c_{\mathrm{w}} a_{3}^{Z \gamma}, \\
\frac{\kappa_{1}^{W W}}{\left(\Lambda_{1}^{W W}\right)^{2}}\left(c_{\mathrm{w}}^{2}-s_{\mathrm{w}}^{2}\right)=\frac{\kappa_{1}^{Z Z}}{\left(\Lambda_{1}^{Z Z}\right)^{2}}+2 s_{\mathrm{w}}^{2} \frac{a_{2}^{\gamma \gamma}-a_{2}^{Z Z}}{m_{Z}^{2}} \\
+2 \frac{s_{\mathrm{w}}}{c_{\mathrm{w}}}\left(c_{\mathrm{w}}^{2}-s_{\mathrm{w}}^{2}\right) \frac{a_{2}^{Z \gamma}}{m_{Z}^{2}}, \\
\frac{\kappa_{2}^{Z \gamma}}{\left(\Lambda_{1}^{Z \gamma}\right)^{2}}\left(c_{\mathrm{w}}^{2}-s_{\mathrm{w}}^{2}\right)=2 s_{\mathrm{w}} c_{\mathrm{w}}\left(\frac{\kappa_{1}^{Z Z}}{\left(\Lambda_{1}^{Z Z}\right)^{2}}+\frac{a_{2}^{\gamma \gamma}-a_{2}^{Z Z}}{m_{Z}^{2}}\right) \\
+2\left(c_{\mathrm{w}}^{2}-s_{\mathrm{w}}^{2}\right) \frac{a_{2}^{Z \gamma}}{m_{Z}^{2}},
\end{gathered}
$$

where $c_{\mathrm{w}}=\cos \theta_{W}, s_{\mathrm{w}}=\sin \theta_{W}, m_{W}$, and $m_{Z}$ are the $W$ and $Z$ boson masses, and $\Delta m_{W}$ is a shift in the $W$ mass. Since $m_{W}$ is measured to high precision [72], we set $\Delta m_{W}=0$, leading to $a_{1}^{Z Z}=a_{1}^{W W}$. The latter relationship also appears under custodial symmetry [73]. Therefore, the set of $13+2$ independent parameters describing the $H V V+H g g$ couplings can be reduced to $8+2$ with the above symmetry relationships.

In our measurements, we further reduce the number of independent parameters in the following way. We assume that the four loop-induced couplings $a_{2,3}^{\gamma \gamma}$ and $a_{2,3}^{Z \gamma}$ are constrained to yield the SM rates of the direct decays $H \rightarrow \gamma \gamma$ and $Z \gamma$. Therefore, in our analysis of EW production and $H \rightarrow 4 \ell$ decay, we set these four couplings to zero because their allowed values are expected to have negligible effect in our coupling measurements. These four anomalous couplings have been tested in our earlier analysis of the $H \rightarrow V V \rightarrow 4 \ell$ process with run 1 data [13] and the obtained constraints were significantly looser than those from the direct decays with on shell photons.
We adopt two approaches to set the relationship between the $H Z Z$ and $H W W$ couplings. The $H W W$ couplings do not contribute to the $H \rightarrow 4 \ell$ decay, but they do contribute to the EW production. In this paper, the relationship between the $H Z Z$ and $H W W$ couplings is mostly relevant for $\mathrm{VBF}$ production with $Z Z$ and $W W$ fusion. There are no kinematic differences between these two processes and it is impossible to disentangle the $H Z Z$ and $H W W$ couplings from these data. We used approach 1 in our previous publications [16-18], where we set $a_{i}^{W W}=a_{i}^{Z Z}$. Approach 2 corresponds to the SMEFT formulation with $\mathrm{SU}(2) \times \mathrm{U}(1)$ symmetry.

In approach 1 , we set the $Z Z$ and $W W$ couplings to be equal, $a_{i}^{W W}=a_{i}^{Z Z}$. Formally, this could be considered as the relationship in Eqs. (3)-(6) in the limiting case $c_{\mathrm{w}}=1$. As a result, we are left with four anomalous couplings to be measured, in addition to the SM-like couplings $a_{1}: a_{2}, a_{3}$, $\kappa_{1} /\left(\Lambda_{1}\right)^{2}$, and $\kappa_{2}^{Z \gamma} /\left(\Lambda_{1}^{Z \gamma}\right)^{2}$, where we drop the $Z Z$ superscript from the couplings. We adopt this approach both for its simplicity and to be able to relate the four anomalous couplings constrained with the $H \rightarrow 4 \ell$ decay to the equivalent four couplings in the pseudo observables approach. This requires an independent measurement of the $\kappa_{2}^{Z \gamma}$ term, which would otherwise be eliminated by the relationship in Eq. (7). Therefore, this approach is slightly less restrictive than the SMEFT formulation adopted in approach 2 discussed below.

In approach 2, we adopt the full set of $\mathrm{SU}(2) \times \mathrm{U}(1)$ symmetry relationships in Eqs. (3)-(7) with $s_{\mathrm{W}}^{2}=0.23119$ [72]. The number of independent $H V V$ parameters is further reduced from five to four: $a_{1}, a_{2}, a_{3}$, and $\kappa_{1} /\left(\Lambda_{1}\right)^{2}$. There is a linear one-to-one relationship between the amplitude couplings in Eq. (2) and the EFT couplings in the Higgs basis in the notation of Refs. [28,33]:

$$
\begin{gathered}
\delta c_{\mathrm{z}}=\frac{1}{2} a_{1}-1, \\
c_{\mathrm{z} \square}=\frac{m_{Z}^{2} s_{\mathrm{w}}^{2}}{4 \pi \alpha} \frac{\kappa_{1}}{\left(\Lambda_{1}\right)^{2}}, \\
c_{\mathrm{zz}}=-\frac{s_{\mathrm{w}}^{2} c_{\mathrm{w}}^{2}}{2 \pi \alpha} a_{2}, \\
\tilde{c}_{\mathrm{zz}}=-\frac{s_{\mathrm{w}}^{2} c_{\mathrm{w}}^{2}}{2 \pi \alpha} a_{3} .
\end{gathered}
$$

Ignoring small loop-induced corrections, the above four parameters are expected to be zero in the SM. Since we set $a_{2,3}^{\gamma \gamma}$ and $a_{2,3}^{Z \gamma}$ to zero, the four corresponding parameters in the EFT Higgs basis $c_{\gamma \gamma}, c_{z \gamma}, \tilde{c}_{\gamma \gamma}$, and $\tilde{c}_{z \gamma}$ are also zero.

In the case of $H g g$ couplings, the two EFT parameters are defined following the notation of Refs. $[28,33]$ as 


$$
\begin{gathered}
c_{g g}=-\frac{1}{2 \pi \alpha_{\mathrm{S}}} a_{2}^{g g}, \\
\tilde{c}_{g g}=-\frac{1}{2 \pi \alpha_{\mathrm{S}}} a_{3}^{g g},
\end{gathered}
$$

where in the SM, $c_{g g}=0$ and $\tilde{c}_{g g}=0$, and the SM process is generated by the quark loop not accounted for in the $a_{2}^{g g}$ coupling. Finally, in the case of the $H$ ff couplings, the $\kappa_{\mathrm{f}}$ and $\tilde{\kappa}_{\mathrm{f}}$ parameters in Eq. (1) can be treated as EFT parameters [28], where in the SM, $\kappa_{\mathrm{f}}=1$ and $\tilde{\kappa}_{\mathrm{f}}=0$.

\section{Parametrization of cross sections}

We use the narrow-width approximation and parametrize differential cross section of the on shell $H$ boson production process $j$ and decay to a final state $f$ following Refs. [28,33] as

$$
\sigma(j \rightarrow H \rightarrow f) \propto \frac{\left(\sum_{i l} \alpha_{i l}^{(j)} a_{i} a_{l}\right)\left(\sum_{m n} \alpha_{m n}^{(f)} a_{m} a_{n}\right)}{\Gamma_{H}},
$$

where $a_{i}$ are the real couplings describing the $H \mathrm{ff}, H g g$, or $H V V$ interactions and include generically the $\kappa_{i}$ in Eqs. (1) and (2). The coefficients $\alpha_{i l}^{(k)}$ are in general functions of kinematic observables for the differential cross section distributions and are modeled with simulation. The total width $\Gamma_{H}$ depends on the couplings $a_{i}$ and potentially on the partial decay width to unobserved or invisible final states, a dependence that must be taken into account when interpreting cross section measurements in terms of couplings.

When we perform the amplitude analysis of the data, in the likelihood based on Eq. (14), we keep all cross terms in the expansion of powers of $\Lambda^{-N}$ with $N=0,2,4,6,8$, where formally each dimension-6 operator, or anomalous $a_{i}$ coupling, receives the $\Lambda^{-2}$ contribution in Eq. (2), even if not explicitly shown, while the SM tree-level coupling carries no such contribution. While this may create inconsistency in the EFT approach with the $\Lambda^{-N}$ terms kept or neglected from the higher-dimension contributions, this allows us to keep the likelihood positive definite, which is an important consideration in the experimental analysis of the data discussed in Sec. V. Because interference contributions may become negative, dropping certain terms in the expansion may lead to negative probability. The importance of $N=4,6,8$ contributions may be considered as testing whether the current precision is sufficient to treat our results within the EFT approach, and we leave this test to the interpretation of the results. However, regardless of EFT validity, our results are presented in a fully selfconsistent formulation of amplitude decomposition, which can either be translated to the EFT interpretation or treated as a test of consistency of the data with the SM, including the search for new sources of $C P$ violation.

\section{Parametrization of the signal strength and cross section fractions}

We present the primary results in terms of cross sections, or equivalently, signal strengths $\mu_{j}=\sigma_{j} / \sigma_{j}^{\mathrm{SM}}$, and the fractional contributions $f_{a i}$ of the couplings $a_{i}$ to cross sections $\left(\sum_{m n} \alpha_{m n} a_{m} a_{n}\right)$ of a given decay process. The ratios of couplings entering Eq. (14) can be expressed through $f_{a i}$, and the common factors, such as the total width $\Gamma_{H}$ and the SM-like coupling squared, are absorbed into the signal strength. This formulation with $\mu_{j}$ and $f_{a i}$ allows the presentation of experimental results in the most direct way, with a minimal and complete set of parameters describing the given processes. This approach has several convenient features. The cross sections and their ratios are invariant with respect to the coupling convention, such as the scaling in Eqs. (8)-(13). The cross section fractions $f_{a i}$ reflect kinematic features in either production or decay in a direct way. They are conveniently bound between -1 and +1 , and most systematic uncertainties cancel in the ratio.

The cross section fraction for $H \mathrm{ff}$ couplings is defined as

$$
f_{C P}^{H \mathrm{ff}}=\frac{\left|\tilde{\kappa}_{\mathrm{f}}\right|^{2}}{\left|\kappa_{\mathrm{f}}\right|^{2}+\left|\tilde{\kappa}_{\mathrm{f}}\right|^{2}} \operatorname{sign}\left(\frac{\tilde{\kappa}_{\mathrm{f}}}{\kappa_{\mathrm{f}}}\right) .
$$

Similarly, the cross section fraction for Hgg couplings is defined as

$$
f_{a 3}^{g g H}=\frac{\left|a_{3}^{g g}\right|^{2}}{\left|a_{2}^{g g}\right|^{2}+\left|a_{3}^{g g}\right|^{2}} \operatorname{sign}\left(\frac{a_{3}^{g g}}{a_{2}^{g g}}\right) .
$$

Both definitions incorporate the relative sign of the possible BSM CP-odd and SM-like CP-even couplings. They are based on the observation that the cross sections of the $H \rightarrow g g$ process are equal for $a_{2}^{g g}=1$ and $a_{3}^{g g}=1$, as are the cross sections of the $H \rightarrow \mathrm{f} \overline{\mathrm{f}}$ process for $\kappa_{\mathrm{f}}=1$ and $\tilde{\kappa}_{\mathrm{f}}=1$ in the limit of $m_{\mathrm{f}} \ll m_{H}$. We note that $f_{a 3}^{g g H}$ is defined following the convention that $a_{2}^{g g}$ and $a_{3}^{g g}$ absorb both pointlike interactions and quark contributions to the loop. Following Ref. [33], the $f_{a 3}^{g g H}$ measurement can also be interpreted in terms of $f_{C P}^{H \mathrm{ff}}$ under the assumption that only the top and bottom quarks contribute to gluon fusion with $\kappa_{t}=\kappa_{b}$ and $\tilde{\kappa}_{t}=\tilde{\kappa}_{b}$ :

$$
\left|f_{C P}^{H \mathrm{ff}}\right|=\left(1+2.38\left[\frac{1}{\left|f_{a 3}^{g g H}\right|}-1\right]\right)^{-1}=\sin ^{2} \alpha^{H \mathrm{ff}},
$$

where the signs of $f_{C P}^{H \mathrm{ff}}$ and $f_{a 3}^{g g H}$ are equal, and $\alpha^{H \mathrm{ff}}$ is an effective parameter sometimes used to describe the $C P$-odd contribution to the $H$ boson Yukawa couplings. A more detailed analysis of the gluon fusion loop could be performed without the assumption that only the top and bottom quarks contribute. 
TABLE I. List of anomalous $H V V$ couplings $a_{i}^{V V}$ considered, the corresponding measured cross section fractions $f_{a i}^{V V}$ defined in Eq. (18), and the translation coefficients $\alpha_{i i} / \alpha_{11}$ in this definition with the relationship $a_{i}^{Z Z}=a_{i}^{W W}$ (approach 1), and with the SMEFT relationship according to Eqs. (3)-(7) (approach 2). In the case of the $\kappa_{1}$ and $\kappa_{2}^{Z \gamma}$ couplings, the numerical values $\Lambda_{1}=\Lambda_{1}^{Z \gamma}=100 \mathrm{GeV}$ are adopted in this calculation to make the coefficients have the same order of magnitude and the negative sign indicates the convention in Eq. (18) adopted earlier [13]. In approach $2, \kappa_{2}^{Z \gamma}$ is a dependent parameter expressed through Eq. (7) and does not require a translation coefficient.

\begin{tabular}{lccc}
\hline \hline Coupling & Fraction & Approach 1 & Approach 2 \\
\hline$a_{i}^{V V}$ & $f_{a i}^{V V}$ & $\alpha_{i i} / \alpha_{11}$ & $\alpha_{i i} / \alpha_{11}$ \\
\hline$a_{3}$ & $f_{a 3}$ & 0.153 & 0.153 \\
$a_{2}$ & $f_{a 2}$ & 0.361 & 6.376 \\
$-\kappa_{1}$ & $f_{\Lambda 1}$ & 0.682 & 5.241 \\
$-\kappa_{2}^{Z \gamma}$ & $f_{\Lambda 1}^{Z \gamma}$ & 1.746 & --- \\
\hline \hline
\end{tabular}

The cross section fractions in the $H V V$ couplings of the $H$ boson to $\mathrm{EW}$ gauge bosons require more parameters. Since in both of our approaches the $H W W$ couplings are expressed through other $a_{i}^{V V}$ couplings following Eqs. (3)-(6), and because we prefer that our definitions not depend on parton distribution functions (PDFs) and other effects that involve measurement uncertainties, we use the $H \rightarrow Z Z / Z \gamma^{*} / \gamma^{*} \gamma^{*} \rightarrow 2 e 2 \mu$ decay process to define the cross section fractions as

$$
f_{a i}^{V V}=\frac{\left|a_{i}^{V V}\right|^{2} \alpha_{i i}^{(2 e 2 \mu)}}{\sum_{j}\left|a_{j}^{V V}\right|^{2} \alpha_{j j}^{(2 e 2 \mu)}} \operatorname{sign}\left(\frac{a_{i}^{V V}}{a_{1}}\right),
$$

where the $\alpha_{i i}^{(2 e 2 \mu)}$ coefficients are introduced in Eq. (14). The numerical values of these coefficients are given in Table I, where they are normalized with respect to the $\alpha_{11}^{(2 e 2 \mu)}$ coefficient, corresponding to the cross section calculated for $a_{1}=1$. The $\alpha_{i i}^{(2 e 2 \mu)}$ are the cross sections for $a_{i}^{V V}=1$, which are different in the two approaches of the coupling relationship as a result of Eq. (7) adopted in approach 2. The cross section fractions in Eq. (18) can be converted to coupling ratios as

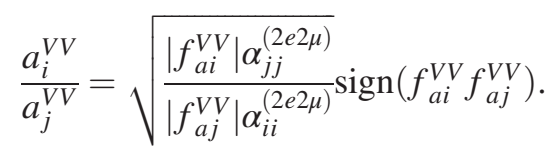

The measured values of $\mu_{j}$ and $f_{a i}$ should be sufficient to adopt them in the fits for EFT parameters jointly with the data from other $H$ boson, top quark, and EW measurements. They allow constraints on the $\kappa_{i}$ and $a_{i}$ couplings in Eqs. (1) and (2). However, it is required to perform a simultaneous measurement of all production and decay channels of the $H$ boson, including unobserved and invisible channels, as they contribute to the total width in Eq. (14). In this paper, we present only a limited interpretation of our data in terms of couplings by making certain assumptions about their relationship. We leave more extensive interpretation to a future combination with other channels.

\section{THE CMS DETECTOR, DATASETS, AND EVENT RECONSTRUCTION}

The $H \rightarrow 4 \ell$ decay candidates are produced in protonproton $(p p)$ collisions at the LHC and are collected and reconstructed in the CMS detector [74]. The data sample used in this analysis corresponds to integrated luminosities of $35.9 \mathrm{fb}^{-1}$ collected in 2016, $41.5 \mathrm{fb}^{-1}$ collected in 2017, and $59.7 \mathrm{fb}^{-1}$ collected in 2018 , for a total of $137 \mathrm{fb}^{-1}$ collected during Run 2 at a $p p$ center-of-mass energy of $13 \mathrm{TeV}$.

The CMS detector comprises a silicon pixel and strip tracker, a lead tungstate crystal electromagnetic calorimeter (ECAL), and a brass/scintillator hadron calorimeter, each composed of a barrel and two end cap sections, all within a superconducting solenoid of $6 \mathrm{~m}$ internal diameter, providing a magnetic field of $3.8 \mathrm{~T}$. Extensive forward calorimetry complements the coverage provided by the barrel and end cap detectors. Outside the solenoid are the gas-ionization detectors for muon measurements, which are embedded in the steel flux-return yoke. A detailed description of the CMS detector can be found in Ref. [74].

Events of interest are selected using a two-tiered trigger system. The first level, composed of custom hardware processors, uses information from the calorimeters and muon detectors to select events at a rate of around $100 \mathrm{KHz}$ within a fixed latency of about $4 \mu \mathrm{s}$ [75]. The second level, known as the high-level trigger, consists of a farm of processors running a version of the full event reconstruction software optimized for fast processing, and reduces the event rate to around $1 \mathrm{KHz}$ before data storage [76].

\section{A. Event reconstruction and selection}

The selection of $4 \ell$ events and associated particles closely follows the methods used in the analyses of the run $1[12,13]$ and run $2[16,17,77,78]$ datasets. The main triggers for the run 2 analysis select either a pair of electrons or muons, or an electron and a muon, passing loose identification and isolation requirements. The transverse momentum $\left(p_{\mathrm{T}}\right)$ for the leading electron or muon is required to be larger than 23 or $17 \mathrm{GeV}$, while that of the subleading lepton is required to be larger than 12 or $8 \mathrm{GeV}$, respectively. To maximize the signal acceptance, triggers requiring three leptons with lower $p_{\mathrm{T}}$ thresholds and no isolation requirement are also used, as well as isolated single-electron and single-muon triggers with thresholds of 27 and $22 \mathrm{GeV}$ in 2016, or 35 and $27 \mathrm{GeV}$ in 2017 and 
2018 , respectively. The overall trigger efficiency for simulated signal events that pass the full selection chain of this analysis is larger than $99 \%$.

Event reconstruction is based on the particle-flow algorithm [79], which exploits information from all the CMS subdetectors to identify and reconstruct individual particles in the event. The particle-flow candidates are classified as charged or neutral hadrons, photons, electrons, or muons, and they are then used to build higher-level objects, such as jets, and to calculate the lepton isolation quantities. Electrons or muons are reconstructed within the geometrical acceptance defined by a requirement on the pseudorapidity $|\eta|<2.5$ or 2.4 and $p_{\mathrm{T}}>7$ or $5 \mathrm{GeV}$, with an algorithm that combines information from the tracker and the ECAL or muon system, respectively. Muons are selected from a list of reconstructed muon track candidates by applying minimal requirements on the track in both the muon system and inner tracker system, and taking into account compatibility with small energy deposits in the calorimeters.

To discriminate between leptons from prompt particle decays and those arising from hadron decays within jets, an isolation variable is calculated for electrons and muons [78]. An isolation requirement is imposed on the muons. Electrons are identified using a multivariate discriminant which includes observables sensitive to the presence of bremsstrahlung along the electron trajectory, the geometrical and momentum-energy matching between the electron trajectory and the associated cluster in the ECAL, the shape of the electromagnetic shower in the ECAL, and variables that discriminate against electrons originating from photon conversions. This discriminant also includes the isolation to suppress electrons originating from EW decays of hadrons within jets [78]. A dedicated algorithm is used to collect the final-state radiation of leptons [77].

The jets are clustered using the anti- $k_{\mathrm{T}}$ jet finding algorithm $[80,81]$ with a distance parameter of 0.4 . The jet momentum is determined as the vector sum of all particle momenta in the jet. Jets must satisfy $p_{\mathrm{T}}>30 \mathrm{GeV}$ and $|\eta|<4.7$ and must be separated from all selected lepton candidates and any selected final-state radiation photons with a requirement on the parameter $\Delta R(\ell / \gamma$, jet $)>0.4$, where $(\Delta R)^{2}=(\Delta \eta)^{2}+(\Delta \phi)^{2}$. Jets are $b$ tagged using the DeepCSV algorithm [82], which combines information about impact parameter significance, the secondary vertex, and jet kinematics.

The reconstructed vertex with the largest value of summed physics-object $p_{\mathrm{T}}^{2}$ is taken to be the primary $p p$ interaction vertex. The physics objects are the jets, clustered using the jet finding algorithm [80,81] with the tracks assigned to candidate vertices as inputs, and the associated missing transverse momentum, taken as the negative vector $p_{\mathrm{T}}$ sum of those jets. In order to suppress muons from in-flight decays of hadrons and electrons from photon conversions, leptons are rejected if the ratio of their impact parameter in three dimensions, computed with respect to the primary vertex position, to their uncertainty is greater than four.

We consider three mutually exclusive lepton flavor channels: $H \rightarrow V V \rightarrow 4 e, 4 \mu$, and $2 e 2 \mu$. At least two leptons are required to have $p_{\mathrm{T}}>10 \mathrm{GeV}$, and at least one is required to have $p_{\mathrm{T}}>20 \mathrm{GeV}$. All four pairs of oppositely charged leptons that can be built with the four leptons are required to satisfy $m_{\ell^{+} \ell^{-}}>4 \mathrm{GeV}$, regardless of lepton flavor, to further suppress events with leptons originating from hadron decays in jet fragmentation or from the decay of low-mass resonances. The $V$ candidates are formed with pairs of leptons of the same flavor and opposite charge that pass the requirement $12<m_{\ell^{+} \ell^{-}}<$ $120 \mathrm{GeV}$, where $m_{1}$ is the invariant mass of the $V$ candidate that is closest to the nominal $Z$ boson mass and $m_{2}$ is the mass of the other one. A value of $m_{1}>40 \mathrm{GeV}$ is required. The reconstructed four-lepton invariant mass, $m_{4 \ell}$, distribution in the region between 70 and $170 \mathrm{GeV}$ is shown in Fig. 6. The $m_{4 \ell}$ region between 105 and $140 \mathrm{GeV}$ is considered in this analysis, which is wide enough to use sidebands for constraining the background normalization in the later fitting procedure [78].

\section{B. Event categorization}

In order to perform a dedicated study of a particular kinematic topology, events are further split into several mutually exclusive categories based on the presence of other particles produced in association with the $H$ boson candidate [78]. Two independent categorization, schemes 1 and 2, discussed below, are followed in this study.

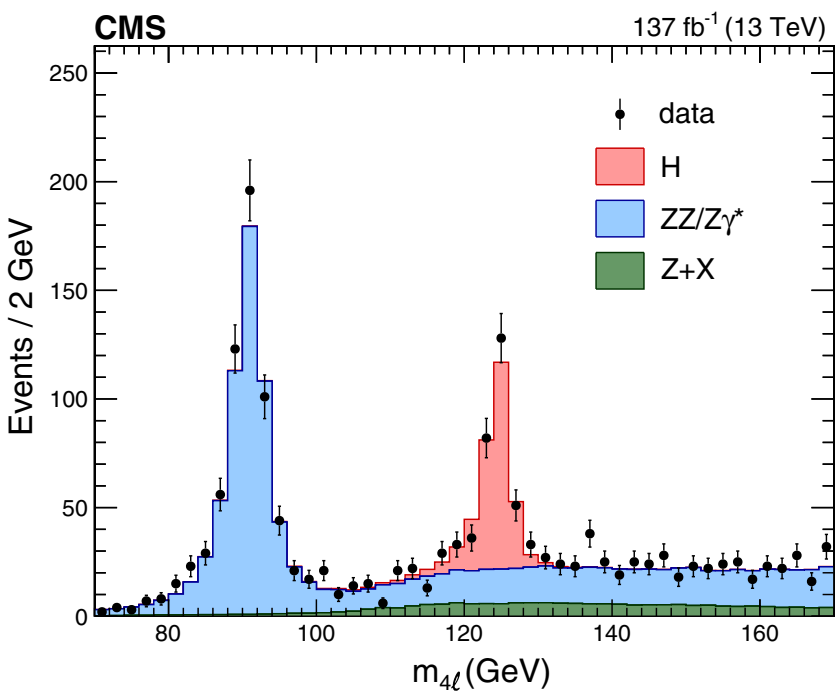

FIG. 6. Four-lepton invariant mass distribution of observed events (data points) and expectation from MC simulation or background estimates (histograms) in the region between 70 and $170 \mathrm{GeV}$ [78]. The peaks from the $Z$ and $H \rightarrow 4 \ell$ decays are visible near 91 and $125 \mathrm{GeV}$, respectively. 
Scheme 1 targets $\mathrm{Htt}$ and $\mathrm{Hgg}$ anomalous couplings, while scheme 2 targets $H V V$ anomalous couplings.

We use the values of kinematic discriminants and other selection requirements to perform the categorization. The definition of these discriminants can be found in Refs. $[16,17,77,78]$ and is further discussed in Sec. IV. They are calculated using the MELA while employing the matrix elements at leading order (LO) in quantum chromodynamics (QCD). These discriminants use full kinematic information from the $H$ boson and from associated jet production and are labeled to indicate a specific topology (1jet, 2jet) and production mechanism (VBF, $W H, Z H$ ), which is discriminated against the dominant gluon fusion process: $\mathcal{D}_{1 \text { jet }}^{\mathrm{VBF}}, \mathcal{D}_{2 \text { jet }}^{\mathrm{VBF}}, \mathcal{D}_{2 \text { jet }}^{Z H}$, and $\mathcal{D}_{2 \mathrm{jet}}^{W H}$. The $\mathcal{D}_{2 \text { jet }}$ discriminants are calculated using both SM and anomalous coupling hypotheses, leading to a set $\mathcal{D}_{2 \text { jet }}^{i}$, all of which are tested in order to maintain high efficiency of VBF and $\mathrm{VH}$ categorization in the presence of anomalous couplings. The discriminants defined for the two-jet topology are illustrated in Fig. 7, where the expected distributions are based on the MC signal simulation discussed in Sec. III C and the background estimate in Sec. III D. To enhance the signal to background ratio in this illustration in Fig. 7, a selection of $\mathcal{D}_{\text {bkg }}>0.7$ is applied. This observable uses information from the lepton kinematic distributions and does not use information from associated jets, as also discussed in Sec. IV.

In categorization scheme 1, the $H t t$ and $H g g$ anomalous couplings are targeted. The categories and selection criteria are identical to the first step of the categorization scheme in Ref. [78] and are optimized to measure the rates of $H$ boson production modes. Because anomalous $\mathrm{Htt}$ and $\mathrm{Hgg}$ couplings have only a small effect on the fractions of $t \bar{t} H$ and $g g H$ events in each category, the optimization based on SM kinematic distributions used for the study in Ref. [78] remains optimal here. The sequential selection criteria in scheme 1 are as follows:

(i) The VBF-2jet category requires exactly four leptons. In addition, there must be either two or three jets of which at most one is $b$ tagged, or at least four jets and no $b$-tagged jets. Finally, $\mathcal{D}_{2 \text { jet }}^{\mathrm{VBF}}>0.5$ is required.

(ii) The $V H$-hadronic category requires exactly four leptons. In addition, there must be either two or three jets, or at least four jets and no $b$-tagged jets. Finally, $\mathcal{D}_{\text {2jet }}^{V H}=\max \left(\mathcal{D}_{\text {2jet }}^{W H}, \mathcal{D}_{2 \text { jet }}^{Z H}\right)>0.5$ is required.

(iii) The $V H$-leptonic category requires no more than three jets and no $b$-tagged jets in the event, and exactly one additional lepton or one additional pair of opposite-sign same-flavor leptons. This category also includes events with no jets and at least one additional lepton.

(iv) The $t \bar{t} H$-hadronic category requires at least four jets, if one is a $b$-tagged jet, and no additional leptons.

(v) The $t \bar{t} H$-leptonic category requires at least one additional lepton in the event.

(vi) The VBF-1jet category requires exactly four leptons, exactly one jet and $\mathcal{D}_{1 \text { jet }}^{\mathrm{VBF}}>0.7$.

(vii) The untagged category consists of the remaining events.

The number of events expected from signal simulation and background estimation are shown along with the observed number of events for each scheme 1 category in Table II.
CMS

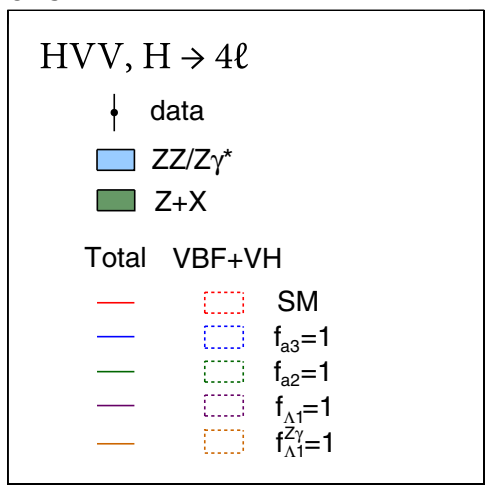

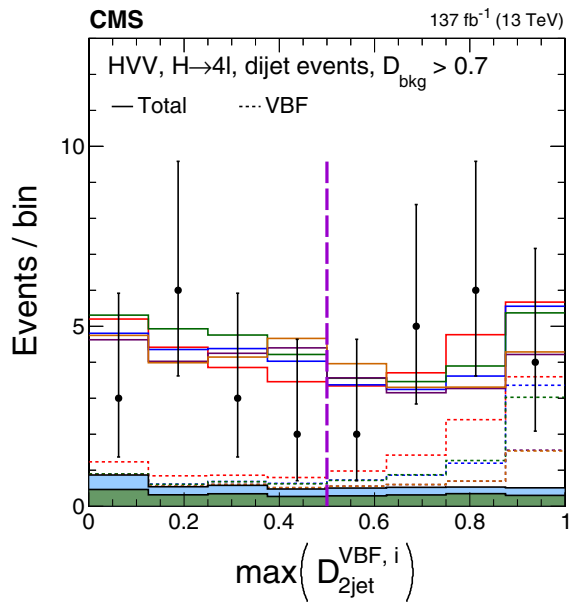

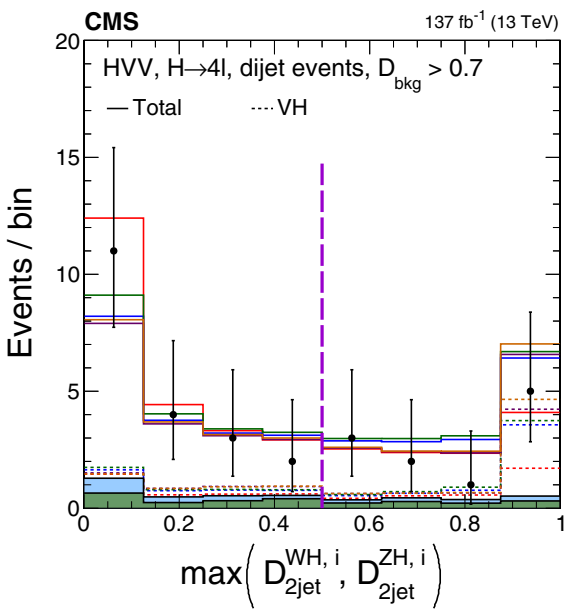

FIG. 7. The distributions of observed events (data points) and expectation (histograms) for $\max \left(\mathcal{D}_{2 \mathrm{jet}}^{\mathrm{VBF}, i}\right)$ (middle) and $\max \left(\mathcal{D}_{2 \text { jet }}^{W H, i}, \mathcal{D}_{2 \text { jet }}^{Z H, i}\right)$ (right), where maximum is evaluated over the SM and the four anomalous coupling hypotheses $i$, described in the legend (left). Only events with at least two reconstructed jets are shown, and the requirement $\mathcal{D}_{\text {bkg }}>0.7$ is applied in order to enhance the signal contribution over the background, where $\mathcal{D}_{\text {bkg }}$ is calculated using decay information only. The expectation is shown for the total distribution, including background and all production mechanisms of the $H$ boson, and for the VBF (middle) and $V H$ (right) signals, which are enhanced in the region above 0.5 , indicated with the vertical dashed line. 
TABLE II. The numbers of events expected in the SM for different $H$ signal (sig) and background (bkg) contributions and the observed number of events in each category defined in scheme 1 targeting $H \mathrm{ff}$ and $H g g$ anomalous couplings. The $t \bar{t} H$ signal expectation is quoted for the SM and anomalous coupling $\left(\kappa_{t}=0, \tilde{\kappa}_{t}=1.6\right)$ scenario, both generated with the same cross section.

\begin{tabular}{|c|c|c|c|c|c|c|c|}
\hline & Untagged & VBF-1jet & VBF-2jet & $V H$-leptonic & $V H$-hadronic & $t \bar{t} H$-leptonic & $t \bar{t} H$-hadronic \\
\hline$g g H$ sig & 182.98 & 15.50 & 6.70 & 0.35 & 4.68 & 0.02 & 0.18 \\
\hline VBF sig & 7.23 & 3.28 & 7.23 & 0.05 & 0.28 & 0.01 & 0.05 \\
\hline$W H$ sig & 2.68 & 0.22 & 0.22 & 1.07 & 1.17 & 0.03 & 0.03 \\
\hline $\mathrm{ZH}$ sig & 2.20 & 0.14 & 0.15 & 0.26 & 0.78 & 0.02 & 0.05 \\
\hline$b \bar{b} H \operatorname{sig}$ & 1.90 & 0.13 & 0.08 & 0.03 & 0.07 & 0.00 & 0.01 \\
\hline $\bar{t} \bar{t} H \operatorname{sig}$ & 0.43 & 0.00 & 0.08 & 0.14 & 0.15 & 0.68 & 0.86 \\
\hline$\left(\tilde{\kappa}_{t}=1.6\right)$ & $(0.45)$ & $(0.00)$ & $(0.12)$ & $(0.15)$ & $(0.15)$ & $(0.87)$ & (1.18) \\
\hline$t H$ sig & 0.14 & 0.01 & 0.10 & 0.04 & 0.03 & 0.04 & 0.03 \\
\hline Total sig & 197.89 & 19.31 & 14.57 & 2.00 & 7.40 & 0.80 & 1.23 \\
\hline$q \bar{q} \rightarrow 4 \ell$ bkg & 210.50 & 6.93 & 1.92 & 2.23 & 1.87 & 0.08 & 0.04 \\
\hline$g g \rightarrow 4 \ell \mathrm{bkg}$ & 19.79 & 1.53 & 0.56 & 0.38 & 0.24 & 0.01 & 0.01 \\
\hline EW bkg & 3.43 & 0.18 & 1.37 & 0.26 & 0.57 & 0.24 & 1.07 \\
\hline$Z+X$ bkg & 77.94 & 2.46 & 4.88 & 1.20 & 3.29 & 0.21 & 1.07 \\
\hline Sig + bkg & 509.55 & 30.41 & 23.30 & 6.05 & 13.38 & 1.33 & 3.41 \\
\hline Observed & 539 & 27 & 20 & 10 & 12 & 0 & 2 \\
\hline
\end{tabular}

In categorization scheme 2 , which targets anomalous $H V V$ couplings, the categorization sequence is modified in three ways in order to be more sensitive to the $H V V$ couplings. First, the $\mathcal{D}_{2 \text { jet }}$ discriminants calculated using the SM hypothesis for $\mathrm{VBF}$ or $V H$ are less sensitive to anomalous $\mathrm{VBF}$ or $\mathrm{VH}$ production, so the selection for the VBF-2jet and $V H$-hadronic categories is modified to be more efficient for BSM hypotheses. Second, the $t \bar{t} H$ categories are dropped and these events are merged into the untagged category; $t \bar{t} H$ forms a small background to $\mathrm{VBF}$ and $V H$. Third, a boosted category is added. This category, adapted from the second (and finer) categorization scheme in Ref. [78], is designed for events where not all associated particles are fully reconstructed, so that the full kinematic information cannot be used to measure anomalous couplings. After these modifications, scheme 2 contains six categories, with sequential selection criteria as follows:

(i) The VBF-2jet category requires exactly four leptons. In addition, there must be either two or three jets of which at most one is $b$ tagged, or at least four jets and no $b$-tagged jets. Finally, $\max \left(\mathcal{D}_{2 \mathrm{jet}}^{\mathrm{VBF}, i}\right)>0.5$ using either the SM or any of the four BSM signal hypotheses $(i)$ for the VBF production is required. See Fig. 7 (middle) for illustration.

(ii) The $V H$-hadronic category requires exactly four leptons. In addition, there must be either two or three jets, or at least four jets and no $b$-tagged jets. Finally, $\max \left(\mathcal{D}_{2 \text { jet }}^{W H, i}, \mathcal{D}_{2 \text { jet }}^{Z H, i}\right)>0.5$ using either the SM or any of the four BSM signal hypotheses $(i)$ for the $V H$ production is required. See Fig. 7 (right) for illustration.

(iii) The $V H$-leptonic category requires no more than three jets and no $b$-tagged jets in the event, and exactly one additional lepton or one additional pair of opposite-sign same-flavor leptons. This category also includes events with no jets and at least one additional lepton.

(iv) The VBF-1jet category requires exactly four leptons, exactly one jet and $\mathcal{D}_{1 \text { jet }}^{\mathrm{VBF}}>0.7$.

(v) The boosted category requires exactly four leptons, three or fewer jets, or at least four jets and no $b$-tagged jets, and the transverse momentum of the four-lepton system $p_{\mathrm{T}}^{4 \ell}>120 \mathrm{GeV}$.

(vi) The untagged category consists of the remaining events.

The number of events expected from signal simulation and background estimation are shown along with the observed number of events for each scheme 2 category in Table III.

The events in each category in either schemes 1 or 2 are further characterized with several observables using the kinematic features of the $H$ boson decay and associated particles, as discussed in Sec. IV.

\section{Monte Carlo simulation}

Monte Carlo simulation is used to model signal processes, which involve the $H$ boson, and background processes in $p p$ interactions at the LHC and their reconstruction in the CMS detector. All MC samples are interfaced with PYTHIA8 [83] to simulate parton showering and multiparton interactions, using version 8.230 for all years with the CUETP8M1 tune [84] for the simulation of the 2016 datataking period, and the CP5 tune [85] for the simulation of the 2017 and 2018 data taking periods. The NNPDF 3.0 parton distribution functions are used [86]. Simulated events include the contribution from additional $p p$ interactions within the same or adjacent bunch crossings (pileup) and are weighted to reproduce the observed pileup 
TABLE III. The numbers of events expected in the SM for different $H$ signal (sig) and background (bkg) contributions and the observed number of events in each category defined in scheme 2 targeting $H V V$ anomalous couplings. The EW (VBF, $W H$, and $Z H$ ) signal expectation is quoted for the SM and four anomalous coupling $\left(a_{3} / a_{2} / \kappa_{1} / \kappa_{2}^{Z \gamma}\right)$ scenarios $f_{a i}=1$, all generated with the same total EW production cross section.

\begin{tabular}{|c|c|c|c|c|c|c|}
\hline & Untagged & Boosted & VBF-1jet & VBF-2jet & $V H$-leptonic & $V H$-hadronic \\
\hline$g g H$ sig & 171.46 & 6.48 & 15.15 & 10.44 & 0.35 & 5.99 \\
\hline VBF sig & 5.06 & 1.18 & 2.64 & 8.60 & 0.06 & 0.54 \\
\hline$\left(a_{3} / a_{2} / \kappa_{1} / \kappa_{2}^{Z \gamma}\right)$ & $\begin{array}{c}(0.29 / 0.29 / \\
0.05 / 0.09)\end{array}$ & $\begin{array}{r}(0.69 / 0.54 / \\
0.52 / 0.48)\end{array}$ & $\begin{array}{c}(0.12 / 0.09 / \\
0.03 / 0.05)\end{array}$ & $\begin{array}{c}(6.10 / 4.95 / \\
1.91 / 1.83)\end{array}$ & $\begin{array}{r}(0.03 / 0.02 / \\
0.01 / 0.01)\end{array}$ & $\begin{array}{c}(0.28 / 0.21 / \\
0.07 / 0.07)\end{array}$ \\
\hline$W H$ sig & 2.18 & 0.43 & 0.29 & 0.22 & 1.11 & 1.20 \\
\hline$\left(a_{3} / a_{2} / \kappa_{1} / \kappa_{2}^{Z \gamma}\right)$ & $\begin{array}{c}(1.93 / 3.15 / \\
0.72 / 0.00)\end{array}$ & $\begin{array}{c}(3.81 / 3.20 / \\
6.28 / 0.00)\end{array}$ & $\begin{array}{c}(0.83 / 0.92 / \\
0.22 / 0.00)\end{array}$ & $\begin{array}{c}(1.20 / 1.05 / \\
2.04 / 0.00)\end{array}$ & $\begin{array}{r}(2.75 / 2.86 / \\
3.47 / 0.00)\end{array}$ & $\begin{array}{c}(3.43 / 3.33 / \\
2.93 / 0.00)\end{array}$ \\
\hline$Z H$ sig & 1.87 & 0.34 & 0.16 & 0.16 & 0.26 & 0.79 \\
\hline$\left(a_{3} / a_{2} / \kappa_{1} / \kappa_{2}^{Z \gamma}\right)$ & $\begin{array}{c}(0.99 / 1.89 / \\
0.68 / 1.17)\end{array}$ & $\begin{array}{c}(1.87 / 1.66 / \\
4.14 / 12.34)\end{array}$ & $\begin{array}{c}(0.30 / 0.35 / \\
0.12 / 0.27)\end{array}$ & $\begin{array}{c}(0.56 / 0.51 / \\
1.30 / 3.88)\end{array}$ & $\begin{array}{c}(0.42 / 0.48 / \\
0.65 / 1.82)\end{array}$ & $\begin{array}{c}(1.42 / 1.53 / \\
1.84 / 4.69)\end{array}$ \\
\hline$b \bar{b} H \operatorname{sig}$ & 1.84 & 0.04 & 0.13 & 0.09 & 0.03 & 0.09 \\
\hline$t \bar{t} H \mathrm{sig}$ & 1.65 & 0.04 & 0.00 & 0.32 & 0.13 & 0.19 \\
\hline$t H$ sig & 0.13 & 0.02 & 0.01 & 0.12 & 0.04 & 0.05 \\
\hline Total sig & 184.1 & 8.5 & 18.4 & 19.8 & 1.9 & 8.8 \\
\hline$\left(a_{3} / a_{2} / \kappa_{1} / \kappa_{2}^{Z \gamma}\right)$ & $\begin{array}{r}(178.2 / 180.3 / \\
176.4 / 176.2)\end{array}$ & $\begin{array}{c}(12.9 / 12.0 / \\
17.5 / 19.4)\end{array}$ & $\begin{array}{c}(16.5 / 16.7 / \\
15.7 / 15.6)\end{array}$ & $\begin{array}{c}(18.7 / 17.4 / \\
16.1 / 16.6)\end{array}$ & $\begin{array}{c}(3.7 / 3.9 / \\
4.6 / 2.3)\end{array}$ & $\begin{array}{c}(11.4 / 11.4 / \\
11.1 / 11.0)\end{array}$ \\
\hline$q \bar{q} \rightarrow 4 \ell \mathrm{bkg}$ & 206.05 & 1.89 & 6.78 & 2.78 & 2.21 & 2.30 \\
\hline$g g \rightarrow 4 \ell \mathrm{bkg}$ & 19.05 & 0.38 & 1.52 & 0.76 & 0.37 & 0.31 \\
\hline EW bkg & 3.50 & 0.66 & 0.20 & 1.98 & 0.23 & 0.85 \\
\hline$Z+X$ bkg & 69.87 & 3.73 & 2.46 & 9.70 & 1.20 & 4.10 \\
\hline $\mathrm{Sig}+\mathrm{bkg}$ & 481.3 & 15.1 & 29.3 & 34.9 & 5.9 & 16.24 \\
\hline$\left(a_{3} / a_{2} / \kappa_{1} / \kappa_{2}^{Z \gamma}\right)$ & $\begin{array}{r}(475.4 / 477.5 / \\
473.6 / 473.4)\end{array}$ & $\begin{array}{c}(19.5 / 18.6 / \\
24.1 / 26.0)\end{array}$ & $\begin{array}{c}(27.4 / 27.6 / \\
26.6 / 26.5)\end{array}$ & $\begin{array}{c}(33.8 / 32.4 / \\
31.1 / 31.6)\end{array}$ & $\begin{array}{c}(7.7 / 7.9 / \\
8.6 / 6.3)\end{array}$ & $\begin{array}{c}(18.83 / 18.78 / \\
18.54 / 18.47)\end{array}$ \\
\hline Observed & 512 & 18 & 27 & 30 & 10 & 13 \\
\hline
\end{tabular}

distribution in data. The MC samples are further processed through a dedicated simulation of the CMS detector based on GEANT4 [87].

The JHUGen7.3.0 [29-33] MC program is used to simulate all anomalous couplings in the $H$ boson production and $H \rightarrow Z Z / Z \gamma^{*} / \gamma^{*} \gamma^{*} \rightarrow 4 \ell$ decay as discussed in Sec. II. The MELA [29-33] package contains a library of matrix elements from JHUGen for the signal and MCFM7.0.1 [88] for the background; these matrix elements are used to apply weights to events in any MC sample to model any other set of anomalous or SM couplings.

The SM production of the $H$ boson through VBFVBF, in association with a $W$ or $Z$ boson, or with a $t t$ pair is simulated using both JHUGen at LO in QCD and POWHEG2 [89-93] at next-to-leading order (NLO) in QCD. Production in association with a $b \bar{b}$ pair or single top quark is simulated at $\mathrm{LO}$ in QCD via JHUGen. In the VBF, $V H$, and $t \bar{t} H$ production modes, the JHUGen and POWHEG simulations are explicitly compared after parton showering in the SM case, and no significant differences are found in kinematic observables. Therefore, the JHUGen simulation is adopted to describe kinematic distributions in the VBF, $V H, t \bar{t} H, t H$, and $b \bar{b} H$ production modes with anomalous couplings, with the expected yields scaled to match the SM theoretical predictions [28] for inclusive cross sections and POWHEG simulation for categorization of events based on associated particles in the SM. There are no observable anomalous effects in kinematic distributions of the $b \bar{b} H$ process [32], but we keep this process in modeling its event contribution. The considered $\mathrm{VH}$ process does not include $g g \rightarrow \mathrm{ZH}$ production, which is expected to contribute about $5 \%$ of the $V H$ cross section and is therefore neglected in this analysis. This process has been studied with JHUGen, including anomalous $H V V$ and $H \mathrm{ff}$ couplings, and it was found that the dependence on anomalous $H V V$ couplings is suppressed [33].

Gluon fusion production is simulated with the POWHEG2 event generator at NLO in QCD. The kinematic features of events produced in gluon fusion with two associated jets are also modified by anomalous $\mathrm{Hgg}$ couplings. These effects are studied using MADGRAPH5_aMC@NLO 2.6.0 $[63,94]$ and JHUGen. Simulation with the MINLO [95] program at NLO in QCD is used for evaluation of systematic uncertainties related to the modeling of two associated jets. The relationship between the $\mathrm{Hff}$ and $\mathrm{Hgg}$ couplings follows JHUGen with the relative sign of $C P$-odd and $C P$-even coefficients opposite to that assumed in 
MADGRAPH5_aMC@NLO2.6.0, as discussed in Ref. [33]. The sign convention of the photon field in JHUGen7.3.0 is opposite to that in MADGRAPH5_aMC@NLO2.6.0, which leads to the opposite sign of the $H Z \gamma$ couplings. This sign convention depends on the sign in front of the gauge fields in the covariant derivative.

In all of the above cases, the subsequent decay $H \rightarrow Z Z / Z \gamma^{*} / \gamma^{*} \gamma^{*} \rightarrow 4 \ell$ is modeled with JHUGen. All signal processes have been generated under the assumption that the $H$ boson mass is $m_{H}=125 \mathrm{GeV}$. This value has been used in calculations in Secs. II and III. However, in the analysis of the data discussed in Secs. IV and VI, the $m_{H}=125.38 \mathrm{GeV}$ value from Ref. [96] is used. The $m_{4 \ell}$ parametrization, the cross sections and the branching fractions of all processes [28] are adjusted according to $m_{H}=125.38 \mathrm{GeV}$, but the effect on other kinematic distributions of the $H$ boson decay products and associated particles is neglected owing to the small difference between the two $m_{H}$ values.

\section{Background modeling}

The main background in this analysis, $q \bar{q} \rightarrow Z Z / Z \gamma^{*} /$ $\gamma^{*} \gamma^{*} \rightarrow 4 \ell$, is estimated from NLO simulation with POWHEG. A fully differential cross section has been computed at next-to-next-to-leading order (NNLO) in QCD [97] and the NNLO/NLO QCD correction is applied as a function of $m_{4 \ell}$. The $g g \rightarrow Z Z / Z \gamma^{*} / \gamma^{*} \gamma^{*} \rightarrow$ $4 \ell$ background process is simulated with MCFM7.0.1 [88,98-100] at LO in QCD. The cross section of this background process is corrected with an NNLO K factor as a function of $m_{4 \ell}$ [101-103], assuming that the signal and background processes have the same correction for higher orders in QCD. The EW background includes the vector boson scattering and $V Z Z$ processes, generated within the JHUGen framework by adopting the MCFM matrix elements for the background processes. The EW background also incorporates other $V V V, t \bar{t} V V$, and $t \bar{t} V$ processes, which are generated with MADGRAPH5_aMC@NLO.

Other background contributions are estimated using control samples in reconstructed data without relying on simulation. Different sources of leptons such as leptons originating from decays of heavy flavor quarks or light mesons may produce additional background to the $H$ boson signal. We denote this background collectively as the $Z+X$ background and employ a data-driven method for its estimation. The same method has been used in the analyses of run $1[12,13]$ and run $2[16,17,77,78]$ datasets. The lepton misidentification rates are first derived using $Z+1 \ell$ control regions with relaxed selection requirements on the third lepton, and the extracted rates are then applied in $Z+2 \ell$ control regions, where the two additional leptons with relaxed selection requirements have the same lepton flavor of equal or opposite charge [78].

\section{KINEMATIC EFFECTS IN PRODUCTION AND DECAY OF THE $H$ BOSON}

Kinematic distributions of particles produced in the $H$ boson decay or in association with the $H$ boson production are sensitive to the quantum numbers and anomalous couplings of the $H$ boson. Four main production topologies are studied: $g g H, \mathrm{VBF}, Z H$, or $W H$, and $t \bar{t} H$ or $t H$, as illustrated in Fig. 8.

In the $H \rightarrow V V \rightarrow 4 \ell$ decay, shown in Fig. 8, lower right, eight observables fully characterize the kinematic distributions of the decay products and the orientation of the decay frame with respect to the production axis $\boldsymbol{\Omega}^{\mathrm{dec}}=$ $\left\{\theta_{1}, \theta_{2}, \Phi, \theta^{*}, \Phi_{1}, m_{1}, m_{2}, m_{4 \ell}\right\}$ [29]. Sets of observables $\boldsymbol{\Omega}^{\text {prod }}$ for the $g g H, \mathrm{VBF}, V H$, and $t \bar{t} H$ production processes are defined in a similar way [31,32], as shown in Fig. 8. As a result, 13 or more kinematic observables can be defined for the associated production process, with subsequent $H$ boson decay to a four-fermion final state. The MELA is designed to reduce the number of observables to a minimum, while retaining all essential information.

\section{A. Kinematic discriminants}

Full kinematic information from each event, using either the $H \rightarrow V V \rightarrow 4 \ell$ decay or associated particles in its production, is extracted using discriminants from matrix element calculations using the MELA package. The discriminants use a complete set of mass and angular input observables $\boldsymbol{\Omega}[29,31,32]$ to describe kinematic distributions at LO in QCD. Full reconstruction of the four-lepton decay chain and associated particles is employed in the matrix element calculation, following the selection chain discussed in Sec. III. Events with partial reconstruction of associated particles are retained in the analysis by using other kinematic observables, such as the transverse momentum of the reconstructed $H$ boson. In the case of the $t \bar{t} H$ topology, where full reconstruction of the full decay chain of the top quarks is a challenging task, an approximation to the matrix element approach is achieved with machine learning [33].

Two types of discriminants are defined for either the production process, the decay process, or the full production + decay process. These discriminants are

$$
\begin{gathered}
\mathcal{D}_{\text {alt }}(\mathbf{\Omega})=\frac{\mathcal{P}_{\text {sig }}(\mathbf{\Omega})}{\mathcal{P}_{\text {sig }}(\boldsymbol{\Omega})+\mathcal{P}_{\text {alt }}(\mathbf{\Omega})}, \\
\mathcal{D}_{\text {int }}(\mathbf{\Omega})=\frac{\mathcal{P}_{\text {int }}(\mathbf{\Omega})}{2 \sqrt{\mathcal{P}_{\text {sig }}(\boldsymbol{\Omega}) \mathcal{P}_{\text {alt }}(\mathbf{\Omega})}},
\end{gathered}
$$

where the probability density $\mathcal{P}$ of a certain process is calculated using the full kinematic description characterized by $\boldsymbol{\Omega}$ for the processes denoted as "sig" for a signal model and "alt" for an alternative model, which could be an alternative $H$ boson production mechanism (used to 

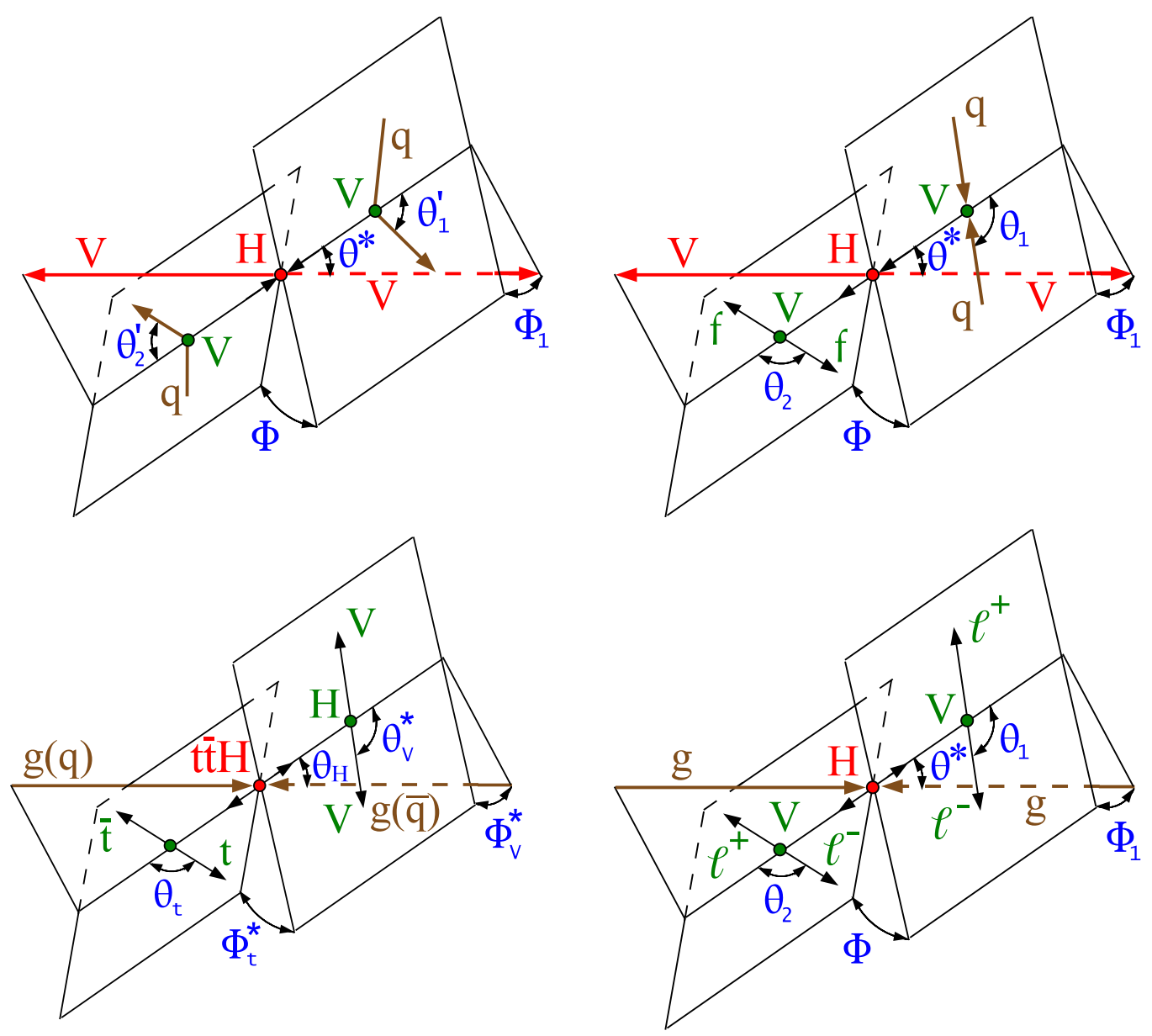

FIG. 8. Four topologies of the $H$ boson production and decay: gluon or EW vector boson fusion $q q \rightarrow V_{1} V_{2}(q q) \rightarrow H(q q) \rightarrow$ $(V V)(q q)$ (upper left); associated production $q q \rightarrow V \rightarrow V H \rightarrow(\mathrm{ff})(V V)$ (upper right); $H$ boson production in association with the top quarks $t \bar{t} H$ or $t H$ (lower left); and four-lepton decay $H \rightarrow V V \rightarrow 4 \ell$ where the incoming gluons $g g$ indicate the collision axis (lower right), and which proceeds either with or without associated particles. The incoming partons are shown in brown and the intermediate or final-state particles are shown in red and green. The angles characterizing kinematic distributions are shown in blue and are defined in the respective rest frames [29,31,32]. The subsequent top quark decay is not shown. See Ref. [32] for details.

categorize events), background (to isolate signal), or an alternative $H$ boson coupling model (to measure coupling parameters). The "int" label refers to the interference between the two model contributions. The probability densities $\mathcal{P}$ are calculated from the matrix elements provided by the MELA package and are normalized to give the same integrated cross section for both processes in the relevant phase space. This normalization leads to a balanced distribution of events in the range between 0 and 1 for the $\mathcal{D}_{\text {alt }}$ discriminants, or between -1 and 1 for $\mathcal{D}_{\text {int }}$. In the special case where the $\mathcal{D}_{\text {int }}$ is calculated between $C P$-even and $C P$-odd models, it is denoted as $\mathcal{D}_{C P}$. The $\mathcal{D}_{C P}$ observable is $C P$ odd, and a forward-backward asymmetry in its distribution would indicate $C P$ violation. This motivates the index " $C P$."

When events are split into the VBF-1/2jet and $V H$ hadronic categories, a set of discriminants $\mathcal{D}_{1 / 2 \text { jet }}$ is constructed, following Eq. (20), where $\mathcal{P}_{\text {sig }}$ corresponds to the signal probability density for the $\mathrm{VBF}$ ( $W H$ or $Z H$ ) production hypothesis in the VBF-tagged ( $\mathrm{VH}$-tagged) category, and $\mathcal{P}_{\text {alt }}$ corresponds to that of $H$ boson production in association with two jets via gluon fusion. When more than two jets pass the selection criteria, the two jets with the highest $p_{\mathrm{T}}$ are chosen for the matrix element calculations. Thereby, the $\mathcal{D}_{1 / 2 \text { jet }}$ discriminants separate the target production mode of each category from gluon fusion production, in all cases using only the kinematic properties of the $H$ boson and two associated jets. The application of the $\mathcal{D}_{1 / 2 \text { jet }}$ discriminants is described in Sec. III, where we introduce four types of discriminants $\mathcal{D}_{1 \text { jet }}^{\mathrm{VBF}}, \mathcal{D}_{2 \mathrm{jet}}^{\mathrm{VBF}, i}, \mathcal{D}_{2 \mathrm{jet}}^{Z H, i}$, and $\mathcal{D}_{2 \text { jet }}^{W H, i}$, with the SM and the four anomalous coupling hypotheses $i$ considered in the signal model.

Several arrays of observables $\vec{x}$ are defined in each category of events, uniquely targeting kinematic features of each category, and are listed in Table IV. One observable, 
TABLE IV. The list of kinematic observables used for category selection and fitting in categorization schemes 1 and 2. Only the main features involving the kinematic discriminants in the category selection are listed, while complete details are given in Sec. III. The untagged category includes the events not selected in the other categories.

\begin{tabular}{|c|c|c|}
\hline Category & Selection & Observables $\vec{x}$ for fitting \\
\hline \multicolumn{3}{|l|}{ Scheme 1} \\
\hline VBF-1jet & $\mathcal{D}_{1 \text { jet }}^{\mathrm{VBF}}>0.7$ & $\mathcal{D}_{\text {bkg }}$ \\
\hline VBF-2jet & $\mathcal{D}_{2 \mathrm{jet}}^{\mathrm{VBF}}>0.5$ & $\mathcal{D}_{\text {bkg }}, \mathcal{D}_{2 \mathrm{jet}}^{\mathrm{VBF}}, \mathcal{D}_{0-}^{g g H}, \mathcal{D}_{C P}^{g g H}$ \\
\hline$V H$-hadronic & $\mathcal{D}_{2 \mathrm{jet}}^{V H}>0.5$ & $\mathcal{D}_{\text {bkg }}$ \\
\hline$V H$-leptonic & see Sec. III & $\mathcal{D}_{\text {bkg }}$ \\
\hline$t \bar{t} H$-hadronic & see Sec. III & $\mathcal{D}_{\text {bkg }}, \mathcal{D}_{0-}^{t \bar{t} H}$ \\
\hline$t \bar{t} H$-leptonic & see Sec. III & $\mathcal{D}_{\text {bkg }}, \mathcal{D}_{0-}^{t \bar{t} H}$ \\
\hline Untagged & None of the above & $\mathcal{D}_{\text {bkg }}$ \\
\hline \multicolumn{3}{|l|}{ Scheme 2} \\
\hline Boosted & $p_{\mathrm{T}}^{4 \ell}>120 \mathrm{GeV}$ & $\mathcal{D}_{\mathrm{bkg}}, p_{\mathrm{T}}^{4 \ell}$ \\
\hline VBF-1jet & $\mathcal{D}_{1 \mathrm{jet}}^{\mathrm{VBF}}>0.7$ & $\mathcal{D}_{\mathrm{bkg}}, p_{\mathrm{T}}^{4 \ell}$ \\
\hline VBF-2jet & $\mathcal{D}_{2 \mathrm{jet}}^{\mathrm{VBF}}>0.5$ & $\mathcal{D}_{\mathrm{bkg}}^{\mathrm{EW}}, \mathcal{D}_{0 \mathrm{~h}+}^{\mathrm{VBF}+\mathrm{dec}}, \mathcal{D}_{0-}^{\mathrm{VBF}+\mathrm{dec}}, \mathcal{D}_{\Lambda 1}^{\mathrm{VBF}+\mathrm{dec}}, \mathcal{D}_{\Lambda 1}^{\mathrm{Z} \gamma, \mathrm{VBF}+\mathrm{dec}}, \mathcal{D}_{\mathrm{int}}^{\mathrm{VBF}}, \mathcal{D}_{C P}^{\mathrm{VBF}}$ \\
\hline$V H$-hadronic & $\mathcal{D}_{2 \mathrm{jet}}^{V H}>0.5$ & $\mathcal{D}_{\text {bkg }}^{\mathrm{EW}}, \mathcal{D}_{0 \mathrm{~h}+}^{\mathrm{VH}+\mathrm{dec}}, \mathcal{D}_{0-}^{\mathrm{VH}+\mathrm{dec}}, \mathcal{D}_{\Lambda 1}^{\mathrm{VH}+\mathrm{dec}}, \mathcal{D}_{\Lambda 1}^{\mathrm{Z \gamma}, \mathrm{VH}+\mathrm{dec}}, \mathcal{D}_{\text {int }}^{V H}, \mathcal{D}_{C P}^{V H}$ \\
\hline$V H$-leptonic & see Sec. III & $\mathcal{D}_{\text {bkg }}, p_{\mathrm{T}}^{4 \ell}$ \\
\hline Untagged & None of the above & $\mathcal{D}_{\mathrm{bkg}}, \mathcal{D}_{0 \mathrm{~h}+}^{\mathrm{dec}}, \mathcal{D}_{0-}^{\mathrm{dec}}, \mathcal{D}_{\Lambda 1}^{\mathrm{dec}}, \mathcal{D}_{\Lambda 1}^{Z \gamma, \mathrm{dec}}, \mathcal{D}_{\mathrm{int}}^{\mathrm{dec}}, \mathcal{D}_{C P}^{\mathrm{dec}}$ \\
\hline
\end{tabular}

$\mathcal{D}_{\text {bkg }}$, is common to most production categories in both schemes 1 and 2. This observable is calculated using Eq. (20) and is designed to separate signal from the dominant background production of four leptons. The $\mathcal{P}_{\text {alt }}$ probability density is calculated for the dominant $q \bar{q} \rightarrow 4 \ell$ background process. The signal and background probability densities include both the matrix element probability based on the four-lepton kinematic properties from MELA and the empirical $m_{4 \ell}$ probability density parametrization extracted from the simulation of detector effects. In the VBF-2jet and $V H$-hadronic categories in scheme 2, the observable $\mathcal{D}_{\mathrm{bkg}}^{\mathrm{EW}}$ is a modified version of $\mathcal{D}_{\text {bkg }}$ which includes the jet information. In this case, $\mathcal{P}_{\text {sig }}$ and $\mathcal{P}_{\text {alt }}$ still include the $m_{4 \ell}$ probability parametrization and four-lepton kinematic information, but they also include kinematic information for the two associated jets. The $\mathcal{P}_{\text {alt }}$ probability density represents the EW and QCD background processes $4 \ell+2$ jets, while $\mathcal{P}_{\text {sig }}$ represents the EW $H$ production processes summed together, VBF, $W H$, and $Z H$. The $\mathcal{D}_{\text {bkg }}$ or $\mathcal{D}_{\text {bkg }}^{\mathrm{EW}}$ calculation employs the SM hypothesis for the signal, while BSM kinematic information is incorporated in the observables discussed next.

\section{B. Observables targeting anomalous $\mathrm{Htt}$ and $\mathrm{Hg}$ g couplings}

In scheme 1, designed to study anomalous $\mathrm{Htt}$ and $\mathrm{Hgg}$ couplings, seven event categories are used. In the untagged, VBF-1jet, $V H$-leptonic, and $V H$-hadronic categories, only one observable $\mathcal{D}_{\mathrm{bkg}}$ is used. These categories do not provide additional information for separating $C P$-even and $C P$-odd contributions in the $\mathrm{Htt}$ and $\mathrm{Hgg}$ couplings, but are included in the fit in order to constrain the rates of the processes. The probability density parametrization of $\mathcal{D}_{\text {bkg }}$ in these categories is not sensitive to the $C P$ structure of either $H \mathrm{ff}$ or $H V V$ interactions.

There is rich kinematic information in $t \bar{t} H$ production because of the sequential decay of the top quarks, as discussed further in Ref. [32]. While it is possible to construct observables, as defined in Eqs. (20) and (21), with matrix element techniques [32], we adopt a machine learning approach to account for partial reconstruction and possible permutations of the jets. A boosted decision tree (BDT) classifier is trained to separate $C P$-even, corresponding to the $\kappa_{t}$ coupling, and $C P$-odd, corresponding to the $\tilde{\kappa}_{t}$ coupling, contributions independently in the $t \bar{t} H$ leptonic and $t \bar{t} H$-hadronic categories. The discriminant $\mathcal{D}_{0-}^{t \bar{t} H}$ is obtained with this approach as the best approximation to Eq. (20), provided the full kinematic information is made available in the calculation [32]. This technique still ensures that the maximal information is retained in the discriminant and is based on the same matrix element used in simulation.

We achieve the full kinematic information in the $\mathcal{D}_{0-}^{i \bar{t} H}$ calculation by including the following observables in the training: the four-momenta of the reconstructed $H$ boson and of the six jets with the largest $p_{\mathrm{T}}$, as well as the $b$-tagging scores of the six jets for resolving their permutation. In addition, in the $\bar{t} H$-leptonic category, the lepton multiplicity and the four-momentum of the highest- $p_{\mathrm{T}}$ lepton not originating from the $H$ boson decay are used as input to the BDT classifier. It is not possible to construct the $\mathcal{D}_{C P}^{\bar{t} t H}$ discriminant, corresponding to Eq. (21), without tagging the flavors of the jets, including distinguishing 

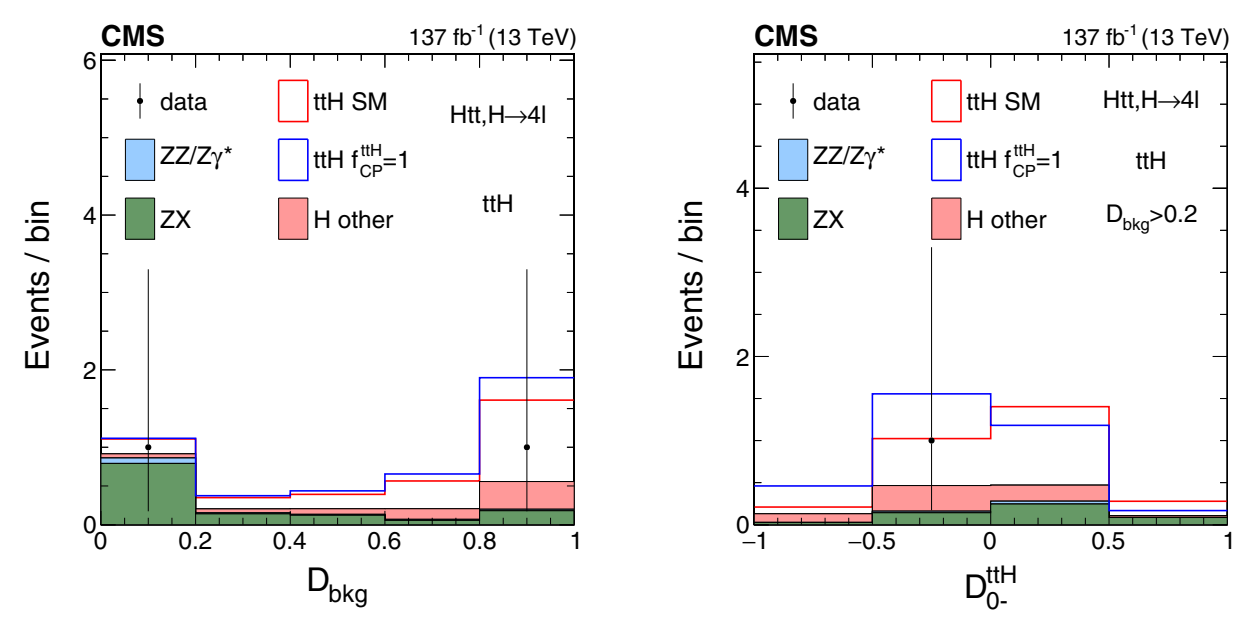

FIG. 9. Distribution of the $\mathcal{D}_{\text {bkg }}$ (left) and $\mathcal{D}_{0-}^{\bar{t} H}$ (right), discriminants in the sum of the $t \bar{t} H$-leptonic and $t \bar{t} H$-hadronic categories in scheme 1. The latter distribution is shown with the requirement $\mathcal{D}_{\text {bkg }}>0.2$ in order to enhance the signal over the background contribution.

quarks from antiquarks [32]. Alternatively, in the leptonic decay of both top quarks one could use the charges of the leptons, but the efficiency of such a method is very low. Therefore, in the two $t \bar{t} H$ categories, two observables are used: $\vec{x}=\left\{\mathcal{D}_{\mathrm{bkg}}, \mathcal{D}_{0-}^{\mathrm{t} t}\right\}$. The distributions of these two discriminants are shown in Fig. 9.

The analysis of gluon fusion production with associated jets is performed in the VBF-2jet category. There are two discriminants that are sensitive to $C P$-even terms, corresponding to the $a_{2}^{g g}$ coupling, and to $C P$-odd terms, corresponding to the $a_{3}^{g g}$ coupling, $\mathcal{D}_{0-}^{g g H}$ and $\mathcal{D}_{C P}^{g g H}$, following Eqs. (20) and (21), respectively. The matrix element for the gluon fusion $H$ boson production in association with two jets includes three possible initial states: quark-quark, quark-gluon, and gluon-gluon. Only the quark-quark initial state is used to calculate these discriminants, because this configuration corresponds to the gluon scattering topology sensitive to $C P$ properties of the $H g g$ coupling [32], by analogy with the weak vector boson scattering process. The jets in the other configurations, quark-gluon and gluon-gluon, are more likely to be initiated from gluon radiation or splitting and are less likely to carry information about the $C P$ properties. For similar reasons, we include the $\mathcal{D}_{2 \text { jet }}^{\mathrm{VBF}}$ discriminant as one of the observables. This discriminant allows us to isolate the VBF-like topology of the events, which is more characteristic of the quark-quark-initiated process.

As a result, in the VBF-2jet category in scheme 1 the observables $\vec{x}=\left\{\mathcal{D}_{\text {bkg }}, \mathcal{D}_{2 \mathrm{jet}}^{\mathrm{VBF}}, \mathcal{D}_{0-}^{g g H}, \mathcal{D}_{C P}^{g g H}\right\}$ are used, as summarized in Table IV. The distributions of the three observables $\mathcal{D}_{\text {bkg }}, \mathcal{D}_{0-}^{g g H}$, and $\mathcal{D}_{C P}^{g g H}$ in this category are shown in Fig. 10, while the $\mathcal{D}_{2 \mathrm{jet}}^{\mathrm{VBF}}$ observable is shown in Fig. 7. It has been shown $[33,44]$ that the azimuthal angle between the two jets carries information similar to $\mathcal{D}_{0-}^{g g H}$ and $\mathcal{D}_{C P}^{g g H}$, but the latter two are better in terms of
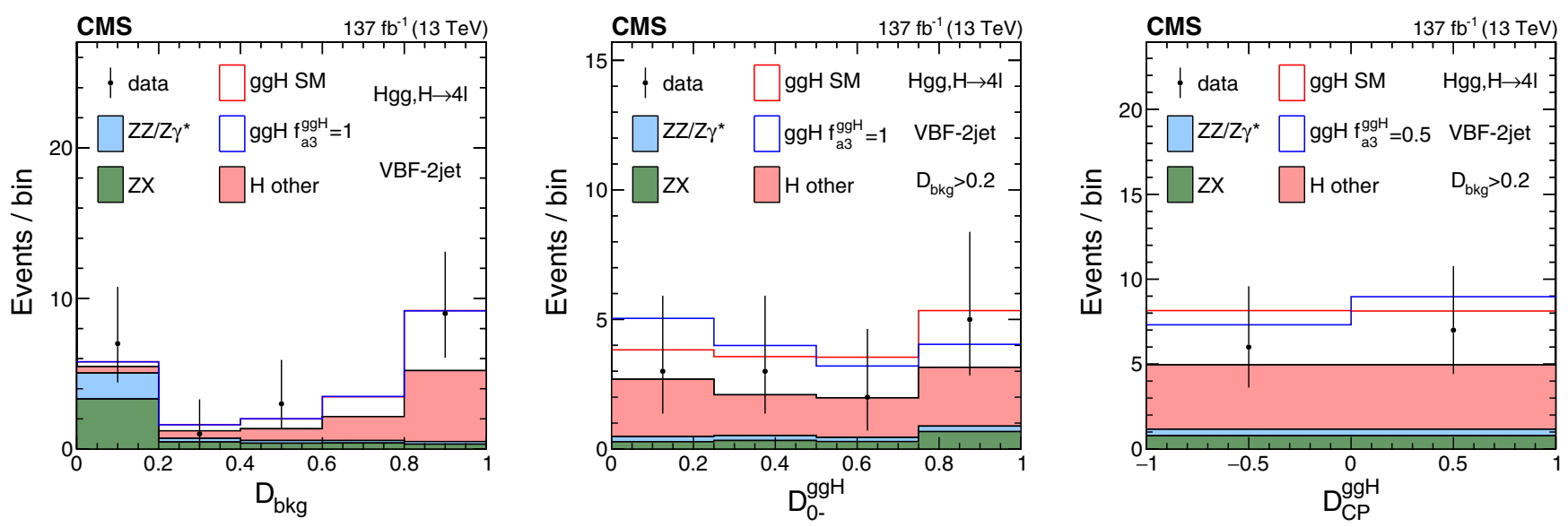

FIG. 10. Distribution of the $\mathcal{D}_{\text {bkg }}\left(\right.$ left), $\mathcal{D}_{0-}^{g g H}$ (middle), and $\mathcal{D}_{C P}^{g g H}$ (right) discriminants in the VBF-2jet category in scheme 1 . The latter two distributions are shown with the requirement $\mathcal{D}_{\text {bkg }}>0.2$ in order to enhance the signal over the background contribution. 
performance and practical application to parametrization in the fit discussed in Sec. V.

\section{Observables targeting anomalous $H V V$ couplings}

In scheme 2, designed to study anomalous $H V V$ couplings, six event categories are used. Two of these categories, VBF 2jet and $V H$ hadronic, target full reconstruction of the associated jets in EW production of the $H$ boson. Therefore, the full matrix element calculation using both production and decay information is employed, as discussed below and summarized in Table IV. Three other categories, boosted, VBF 1 jet, and $V H$ leptonic, also target EW production, but without full reconstruction of the associated particles. Therefore, in these categories matrix element calculations are not employed, and instead the transverse momentum of the $H$ boson candidate $p_{\mathrm{T}}^{4 \ell}$ is used as the second observable. Anomalous couplings in EW production lead to a harder $p_{\mathrm{T}}^{4 \ell}$ spectrum. Finally, in the untagged category, dominated by the $g g H$ events without two associated jets, matrix element calculations using $H \rightarrow V V \rightarrow 4 \ell$ decay information are employed.

Since we target four anomalous $H V V$ couplings appearing in Eq. (2), namely $a_{2}, a_{3}, \kappa_{1} /\left(\Lambda_{1}\right)^{2}$, and $\kappa_{2}^{Z_{\gamma}} /\left(\Lambda_{1}^{Z \gamma}\right)^{2}$, optimal analysis of the $H \rightarrow V V \rightarrow 4 \ell$ decay requires four discriminants of the type given by Eq. (20) and four discriminants of the type given by Eq. (21). In the untagged category, the former four discriminants are defined as $\mathcal{D}_{0 \mathrm{~h}+}^{\mathrm{dec}}$, $\mathcal{D}_{0-}^{\text {dec }}, \mathcal{D}_{\Lambda 1}^{\text {dec }}$, and $\mathcal{D}_{\Lambda 1}^{Z \gamma \text {,dec }}$, respectively, where the index "dec" indicates that only the four-lepton decay information is used. Among the latter four interference discriminants, it was found that the two discriminants corresponding to the $\kappa_{1} /\left(\Lambda_{1}\right)^{2}$ and $\kappa_{2}^{Z \gamma} /\left(\Lambda_{1}^{Z \gamma}\right)^{2}$ couplings are strongly correlated with $\mathcal{D}_{\Lambda 1}^{\text {dec }}$ and $\mathcal{D}_{\Lambda 1}^{Z \gamma \text { dec }}$, and therefore these two interference discriminants are not used. This observation follows from the fact that the $a_{1}$ and $\kappa_{1} /\left(\Lambda_{1}\right)^{2}$ or $\kappa_{2}^{Z \gamma} /\left(\Lambda_{1}^{Z \gamma}\right)^{2}$ couplings correspond to the same tensor structure in Eq. (2) and differ only in the $q_{V}^{2}$ dependence. The remaining two interference discriminants, $\mathcal{D}_{\mathrm{int}}^{\mathrm{dec}}$ and $\mathcal{D}_{C P}^{\mathrm{dec}}$, corresponding to the $a_{2}$ and $a_{3}$ alternative couplings, respectively, are employed in the fit.

In the VBF-2jet and $V H$-hadronic categories, the system of six discriminants discussed above is extended to include both production and decay information, because these categories allow full reconstruction of associated particles. The same four types of discriminants of the untagged category following Eq. (20) are used, namely $\mathcal{D}_{\mathrm{Oh}+}^{\mathrm{VBF}+\text { dec }}$, $\mathcal{D}_{0-}^{\mathrm{VBF}+\mathrm{dec}}, \mathcal{D}_{\Lambda 1}^{\mathrm{VBF}+\mathrm{dec}}$, and $\mathcal{D}_{\Lambda 1}^{\mathrm{Z}, \mathrm{VBF}+\mathrm{dec}}$ in the VBF-2jet category, and $\mathcal{D}_{0 \mathrm{~h}+}^{\mathrm{VH}+\mathrm{dec}}, \quad \mathcal{D}_{0-}^{\mathrm{VH}+\mathrm{dec}}, \quad \mathcal{D}_{\Lambda 1}^{\mathrm{VH}+\mathrm{dec}}, \quad$ and $\mathcal{D}_{\Lambda 1}^{Z \gamma, \mathrm{VH}+\text { dec }}$ in the VH-hadronic category. Here the index "VBF+dec" or "VH+dec" indicates that both production and decay information is used, which means that the kinematic information from the associated jets and the four leptons are utilized in the VBF or $V H$ matrix element calculations. In the case of the $V H$ process, the matrix elements of the $W H$ and $\mathrm{ZH}$ processes are summed. There are more interference discriminants in cases where anomalous couplings appear both in production and decay. However, using interference discriminants with production information only following Eq. (21) is the better approach if one has to limit the number of discriminants. Therefore, $\mathcal{D}_{\text {int }}^{\mathrm{VBF}}$ and $\mathcal{D}_{C P}^{\mathrm{VBF}}$ are used in the VBF-2jet category, and $\mathcal{D}_{\text {int }}^{V H}$ and $\mathcal{D}_{C P}^{V H}$ are used in the $V H$-hadronic category.

Distributions of events for the observables $\vec{x}$ in scheme 2 are illustrated in Figs. 11-13. Here and in Figs. 9 and 10 the expected distributions are based on signal MC simulation discussed in Sec. IIIC and the background estimate in Sec. III D, where cross sections of all processes, including those with the BSM couplings, are set to the SM expectations. The full list of kinematic observables employed in the fit in each category is summarized in the third column of Table IV.

\section{IMPLEMENTATION OF FITTING AND ASSOCIATED UNCERTAINTIES}

In the analysis of the $H \mathrm{ff}, H g g$, or $H V V$ anomalous couplings, the events are split into a total of 63 (in scheme 1, designed to study $H \mathrm{ff}$ and $H g g$ ) or 54 (in scheme 2, designed to study $H V V$ ) categories according to the seven or six production categories, three lepton flavor combinations $(4 e, 4 \mu$, and $2 e 2 \mu)$, and three data periods $(2016,2017$, and 2018). Each event is characterized by its discrete category $k$ and set of input observables $\vec{x}$, as discussed in detail in Sec. IV and summarized in Table IV. The observed distributions of events across the discriminants $\vec{x}$ are illustrated in Figs. 9-13 and are compared to the expected distributions in SM and BSM. However, quantitative characterization of these distributions requires a careful analysis of the multidimensional space of observables $\vec{x}$ and categories $k$, which is discussed below. Preparation of this analysis was performed in a blind way, which means that observed distributions of events were not examined until all details of the fit discussed below were finalized.

\section{A. Likelihood parametrization}

We perform an extended maximum likelihood fit [104] in which the probability density is normalized to the total event yield in each category $k$ as a sum over all signal processes $j$ and background processes $i$ according to

$$
\mathcal{P}_{k}(\vec{x})=\sum_{j} \mu_{j} \mathcal{P}_{j k}^{\mathrm{sig}}\left(\vec{x} ; \vec{\xi}_{j k}, \vec{f}_{j}\right)+\sum_{i} \mathcal{P}_{i k}^{\mathrm{bkg}}\left(\vec{x} ; \vec{\xi}_{i k}\right),
$$

where $\mu_{j}$ is the ratio of the observed cross section to the SM expectation, $\vec{f}_{j}$ is the set of unconstrained parameters describing kinematic distributions in a given process, defined in Eqs. (15), (16), (18), and $\vec{\xi}_{j k}$ are the constrained 

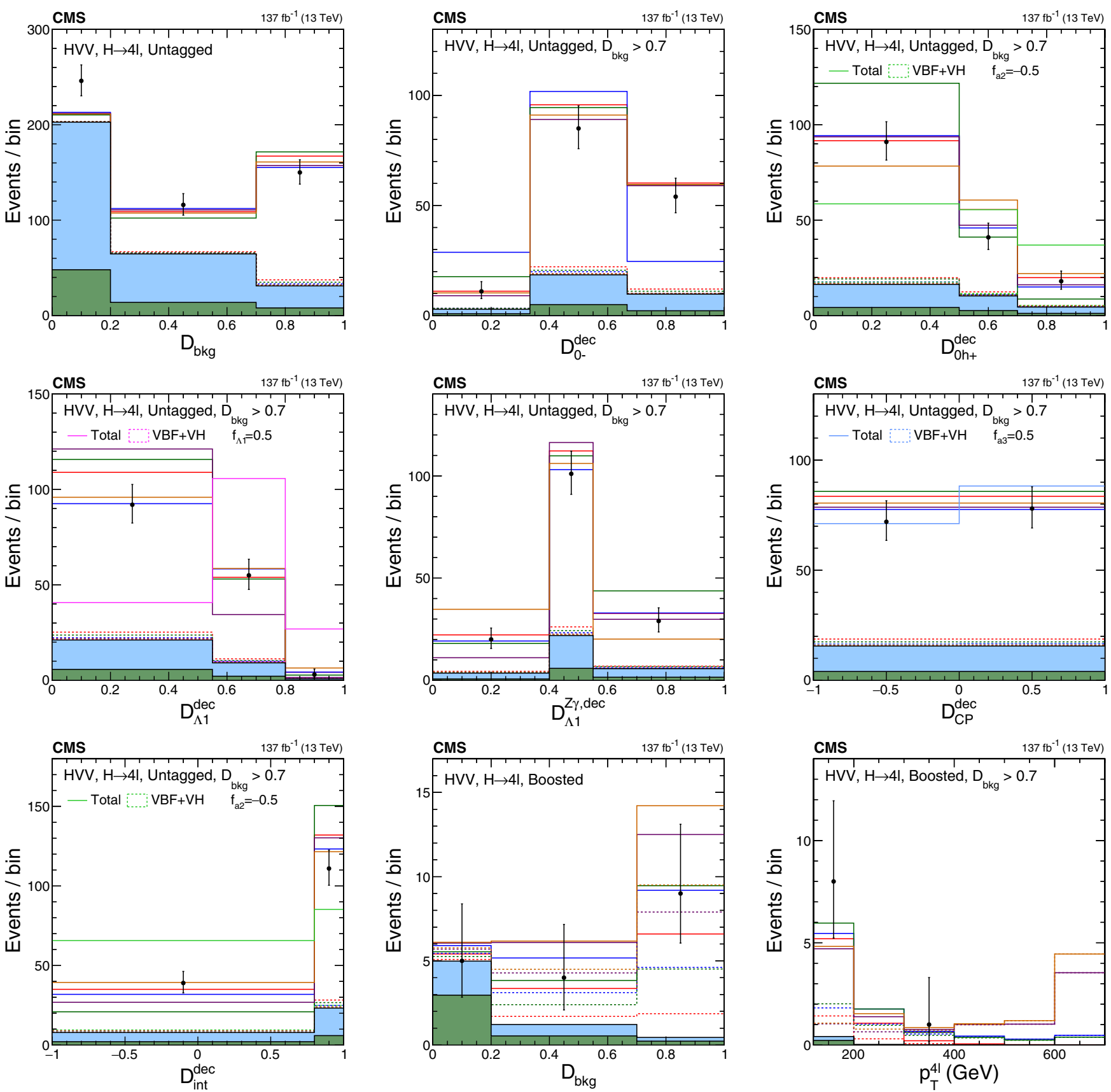

FIG. 11. Distributions of events in the observables used in categorization scheme 2. The first seven plots are in the untagged category: the upper left plot shows $\mathcal{D}_{\text {bkg }}$. The other distributions are shown with the requirement $\mathcal{D}_{\text {bkg }}>0.7$ in order to enhance the signal over the background contribution: $\mathcal{D}_{0-}^{\text {dec }}$ (upper middle), $\mathcal{D}_{0 h+}^{\text {dec }}$ (upper right), $\mathcal{D}_{\Lambda 1}^{\text {dec }}$ (middle left), $\mathcal{D}_{\Lambda 1}^{Z \gamma \text {,dec }}$ (middle middle), $\mathcal{D}_{C P}^{\text {dec }}$, and $\mathcal{D}_{\text {int }}^{\text {dec }}$. The last two plots are shown in the boosted category: $\mathcal{D}_{\text {bkg }}$ (lower middle) and $p_{\mathrm{T}}^{4 \ell}$ with the requirement $\mathcal{D}_{\text {bkg }}>0.7$ and overflow events included in the last bin (lower right). Observed data, background expectation, and five signal models are shown in the plots, as indicated in the legend in Fig. 7 (left). In several cases, a sixth signal model with a mixture of the SM and BSM couplings is shown and is indicated in the legend explicitly.

nuisance parameters reflecting the uncertainties in the above parametrization.

In the case of the gluon fusion process, $\mu_{j}=\mu_{g g H}$ and $\vec{f}_{j}=f_{a 3}^{g g H}$. The dependence on the $C P$-sensitive parameter $f_{a 3}^{g g H}$ appears only in the VBF-2jet category where correlation of the two associated jets is explored. Therefore, in this category a dedicated simulation of the $H$ boson production with two associated jets with MADGRAPH5_aMC@NLO is used, and checked against the JHUGen and MINLO simulation. The interpretation of this process in terms of fermion couplings appearing in the 

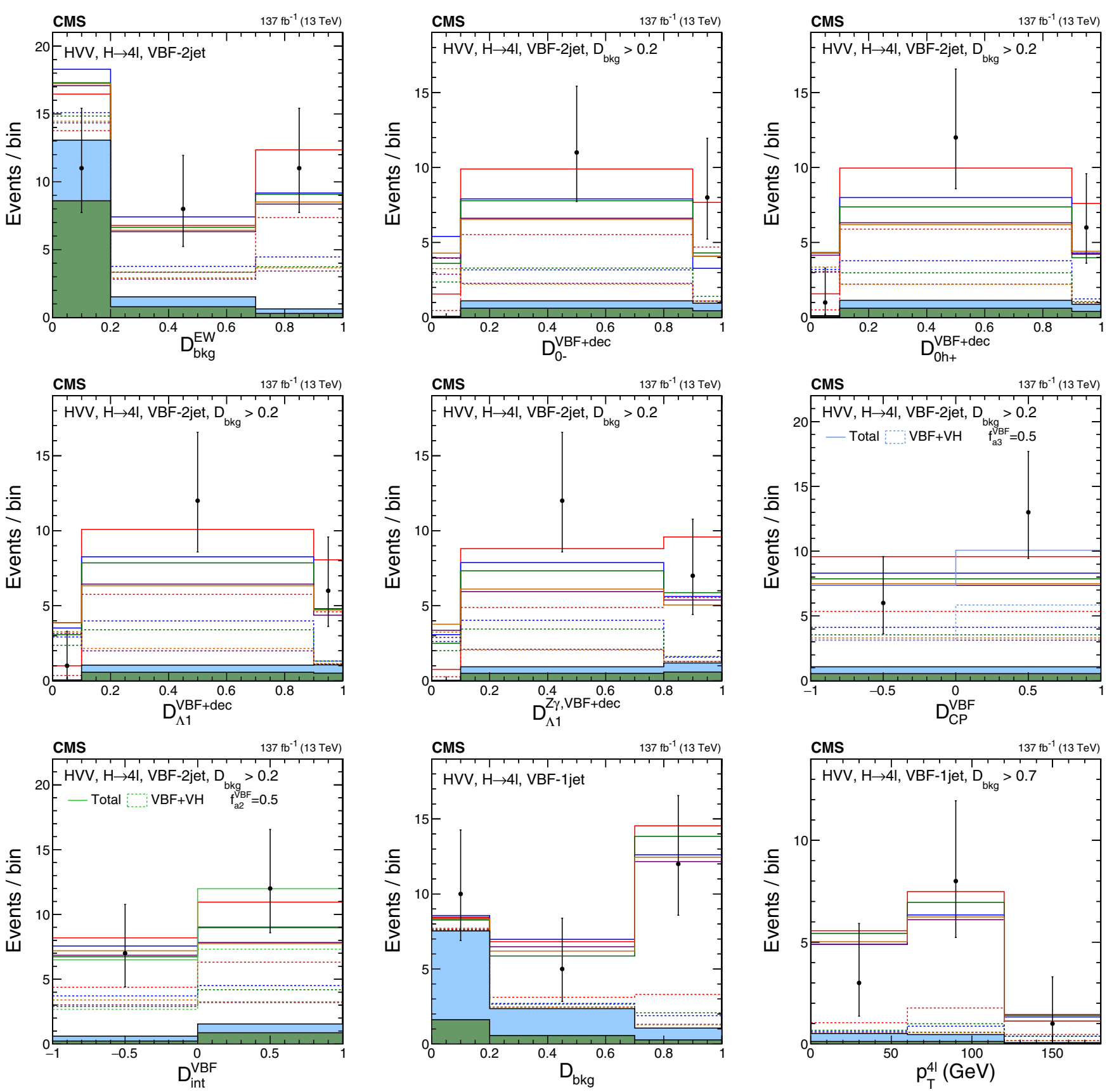

FIG. 12. Distributions of events in the observables used in categorization scheme 2. The first seven plots are in the VBF-2jet category: The upper left plot shows $\mathcal{D}_{\mathrm{bkg}}^{\mathrm{EW}}$. The other distributions are shown with the requirement $\mathcal{D}_{\mathrm{bkg}}^{\mathrm{EW}}>0.2$ in order to enhance the signal over the background contribution: $\mathcal{D}_{0-}^{\mathrm{VBF}+\mathrm{dec}}$ (upper middle), $\mathcal{D}_{0 h+}^{\mathrm{VBF}+\mathrm{dec}}$ (upper right), $\mathcal{D}_{\Lambda 1}^{\mathrm{VBF}+\mathrm{dec}}$ (middle left), $\mathcal{D}_{\Lambda 1}^{\mathrm{Z} \gamma, \mathrm{VBF}+\mathrm{dec}}$ (middle middle), $\mathcal{D}_{C P}^{\mathrm{VBF}}$, and $\mathcal{D}_{\text {int }}^{\mathrm{VBF}}$. The last two plots are shown in the VBF-1jet category: $\mathcal{D}_{\text {bkg }}$ (lower middle) and $p_{\mathrm{T}}^{4 \ell}$ with the requirement $\mathcal{D}_{\text {bkg }}>0.7$ and overflow events included in the last bin (lower right). Observed data, background expectation, and five signal models are shown in the plots, as indicated in the legend in Fig. 7 (left). In several cases, a sixth signal model with a mixture of the SM and BSM couplings is shown and is indicated in the legend explicitly.

gluon fusion loop is discussed in Sec. VI C. In cases where the SM fermions are assumed to dominate the gluon fusion loop, the $\mu_{g g H}$ and $f_{a 3}^{g g H}$ parameters are correlated to $\mu_{\bar{t} \bar{H} H}$ and $f_{C P}^{H t t}$ in the $t \bar{t} H$ process through Eq. (17). A more general case, when both SM fermions and heavy BSM particles contribute to the loop, is also considered. In all cases the relationship between the $H \mathrm{ff}$ and $H g g$ couplings follows JHUGen with the relative sign of $C P$ odd and $C P$-even coefficients opposite to that assumed in MADGRAPH5_aMC@NLO, as discussed in Ref. [33]. 

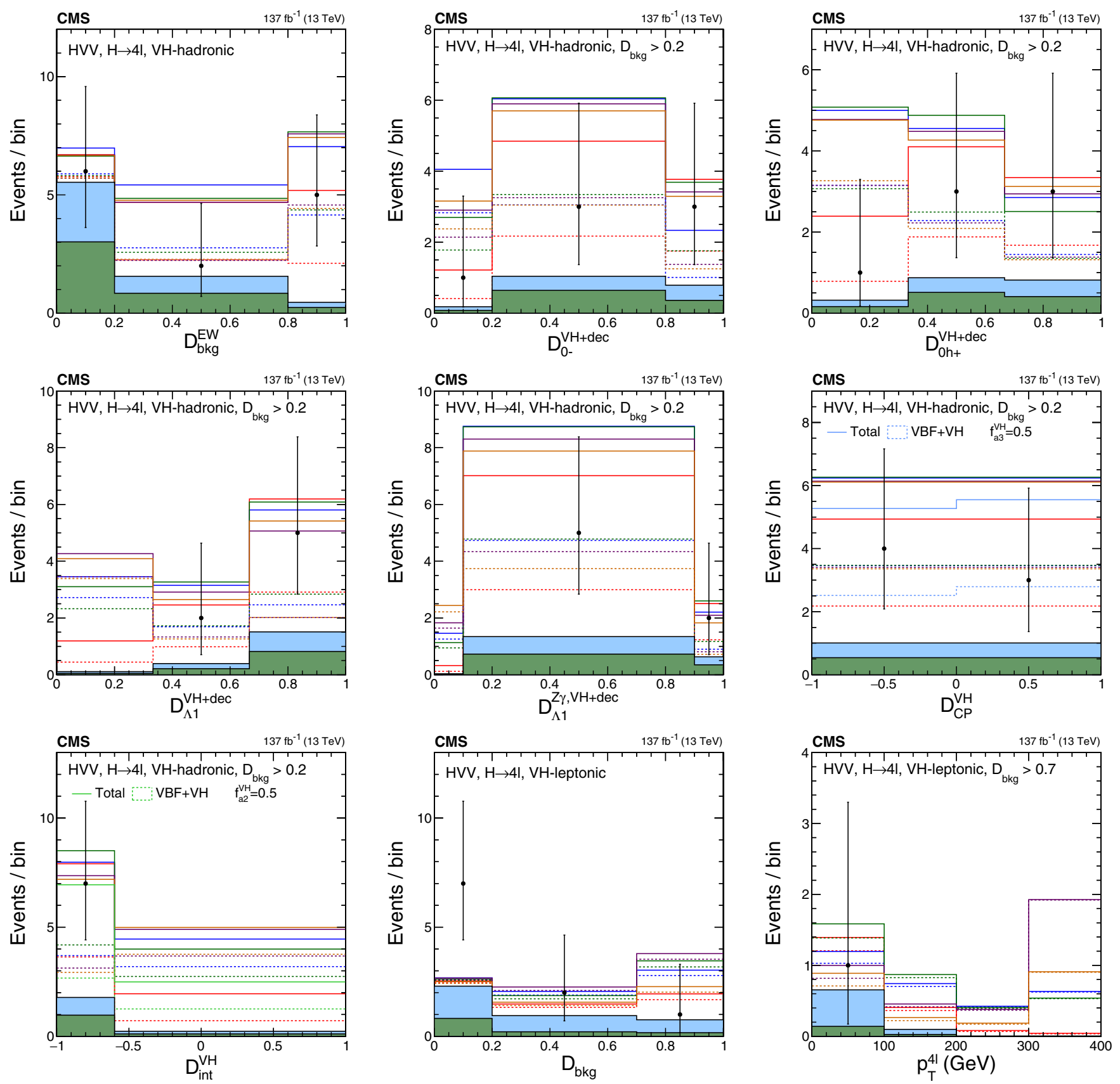

FIG. 13. Distributions of events in the observables used in categorization scheme 2. The first seven plots are in the $V H$-hadronic category: the upper left plot shows $\mathcal{D}_{\text {bkg }}^{\mathrm{EW}}$. The other distributions are shown with the requirement $\mathcal{D}_{\text {bkg }}^{\mathrm{EW}}>0.2$ in order to enhance the signal over the background contribution: $\mathcal{D}_{0-}^{V H+\text { dec }}$ (upper middle); $\mathcal{D}_{0 h+}^{V H+\text { dec }}$ (upper right); $\mathcal{D}_{\Lambda 1}^{V H+\text { dec }}$ (middle left); $\mathcal{D}_{\Lambda 1}^{Z \gamma, V H+\text { dec }}$ (middle middle); $\mathcal{D}_{C P}^{V H}$, and $\mathcal{D}_{\text {int }}^{V H}$. The last two plots are shown in the $V H$-leptonic category: $\mathcal{D}_{\text {bkg }}$ (lower middle) and $p_{\mathrm{T}}^{4 \ell}$ with the requirement $\mathcal{D}_{\text {bkg }}>0.7$ and overflow events included in the last bin (lower right). Observed data, background expectation, and five signal models are shown in the plots as indicated in the legend in Fig. 7 (left). In several cases, a sixth signal model with a mixture of the SM and BSM couplings is shown and is indicated in the legend explicitly.

In the case of the $t \bar{t} H$ process, $\mu_{j}=\mu_{t \bar{t} H}$ and $\vec{f}_{j}=f_{C P}^{H t t}$. The $t H$ production is expected to contribute about $5 \%$ of the total $t \bar{t} H$ signal in this analysis, as shown in Table II, and, therefore, the exact treatment of this process is currently not important. This production mode depends on both $H V V$ and $H \mathrm{ff}$ couplings. The anticipated small yield may be somewhat larger if the expected destructive interference between the $H V V$ and $H \mathrm{ff}$ couplings in the $t H$ process becomes constructive owing to a modification of these couplings. In this analysis, we also introduce a 
possible $C P$-odd Yukawa coupling in the $t H$ process. We fix the sign of the $a_{1}$ coupling to be positive, and so the sign of the $\kappa_{t}$ coupling allows us to float the relative sign of the $H V V$ and $H$ ff contributions in this process. In Scheme 1, we parametrize the $t H$ signal strength with the parameters $\mu_{\bar{t} \bar{H} H}, f_{C P}^{H t t}$, and $\mu_{V}$, defined below. There is a weak dependence of the $\mathcal{D}_{0-}$ distributions on $f_{C P}^{H t t}$ in this channel, which we conservatively neglect. We also neglect anomalous $H V V$ couplings in the $t H$ parametrization because their effect is negligible with the current constraints from analysis of the VBF and $V H$ events. In scheme 2, where we do not have a dedicated category for the top quark coupling measurements, we neglect the dependence of the $t H$ process on the $H V V$ couplings because this would have a negligible effect on the results and unnecessarily complicate the fit parametrization.

For EW processes (VBF, $\mathrm{ZH}, W H$ ), the common unconstrained parameters of interest are $\mu_{j}=\mu_{V}$ and $\vec{f}_{j}=$ $\left(f_{a 3}, f_{a 2}, f_{\Lambda 1}, f_{\Lambda 1}^{Z \gamma}\right)$ in approach 1 or $\vec{f}_{j}=\left(f_{a 3}, f_{a 2}, f_{\Lambda 1}\right)$ in approach 2, when using categorization scheme 2 for fitting $H V V$ couplings. We simplify treatment of the EW processes in categorization scheme 1 when fitting for $\mathrm{CP}$-violating $\mathrm{Hgg}$ or $\mathrm{Htt}$ couplings and allow only the $C P$-violating $f_{a 3}$ parameter in $\vec{f}_{j}$. When all anomalous couplings are set to zero, the signal strength $\mu_{V}$ is equal to the ratio of the cross sections of all EW processes (VBF, $Z H, W H)$ to the SM expectation. In the case of $f_{a i}=1$, this ratio is corrected by the factor $\left(\alpha_{i i} / \alpha_{11}\right)$ as quoted in Table I for decay cross sections, and the inverse of this factor for the EW production cross sections, due to the evolution of the cross sections with anomalous couplings.

It has been shown that there is no sensitivity to the anomalous couplings in the $b \bar{b} H$ process [32] and it is parametrized with the signal strength $\mu_{j}=\mu_{b \bar{b} H}$. Depending on the fit implementation discussed in Sec. VI, this signal strength may be correlated with those in other channels, such as $\mu_{g g H}$ or $\mu_{t \bar{t} H}$. The exact treatment of this process is expected to have negligible effect on the results, because it cannot be distinguished kinematically from other dominant processes and its relative contribution in each kinematic region is expected to be negligibly small, as shown in Tables II and III.

The background processes $i$ in Eq. (22) include the $q \bar{q} \rightarrow 4 \ell, g g \rightarrow 4 \ell$, and EW processes, all of which are estimated with simulation, but receive additional constraints from sidebands in data. The EW background includes vector boson scattering and $V V V$ processes, which are the background counterparts of the $\mathrm{VBF}$ and $V H$ processes. We also include the $t \bar{t} V V$ and $t \bar{t} V$ processes in this background contribution, which are important in the study of the $t \bar{t} H$ signal process. Interference of the signal and background processes is negligible in the analysis of the on-shell $H$ boson production. The $Z+\mathrm{X}$ background contribution models $Z+$ jets and other related processes with lepton misidentification and is estimated from the control regions in the data as discussed in Sec. III D. All signal $j$ and background $i$ processes contributing to Eq. (22) and their expected yields are shown in Tables II and III.

The signal and background probability distributions $\mathcal{P}_{j k}^{\text {sig }}$ and $\mathcal{P}_{i k}^{\mathrm{bkg}}$ appearing in Eq. (22) are binned multidimensional histograms (templates) of observables $\vec{x}$ listed in Table IV. The binning of these templates has been optimized for memory and speed of computer calculations, expected population of events across those bins, and retaining kinematic information. In particular, the large number of discriminants used in the untagged, VBF-2jet, and $\mathrm{VH}$-hadronic categories in scheme 2, requires careful optimization of the binning employed in analysis. In these categories, two bins are used in the two interference discriminants and three bins are used in the other five discriminants, which corresponds to a total of 972 bins in the seven-dimensional (7D) distribution. However, the bins with a very low yield of expected events for all contributions are merged, and the expected symmetry in the distribution of the $\mathcal{D}_{C P}$ observable is enforced. As a result, the total number of independent bins depends on the category, but does not exceed 400 in any of the categories. Even though only a limited number of bins is used in each dimension, the 7D distribution retains substantial kinematic information that is nearly optimal for all anomalous couplings targeted in this analysis. This has been validated against a dedicated analysis targeting one anomalous coupling at a time with a much larger number of bins in each dimension for a smaller number of discriminants, as employed in Refs. [16,17]. This nearly optimal performance is realized in large respect due to the optimal population of events across the range of discriminant values by construction of the MELA.

The $\mathcal{P}_{j k}^{\text {sig }}$ and $\mathcal{P}_{i k}^{\mathrm{bkg}}$ probabilities depend on the parameters $\vec{\xi}_{j k}$ and $\vec{f}_{j}$ and are therefore interpolated between various templates as a function of these parameters. The $\vec{\xi}_{j k}$ reflect systematic uncertainties either in the normalization or shape of both signal and background templates and an analytical linear interpolation is adopted. The $\vec{f}_{j}$ parameters require nontrivial analytical interpolation of the signal templates, which is discussed in more detail below.

\section{B. Signal parametrization}

In the signal production and decay processes, the same anomalous couplings could appear either on both the production and decay sides simultaneously, as in the case of $H V V$ couplings in EW production and $H \rightarrow V V \rightarrow 4 \ell$ decay, or only on one side (production or decay). This is illustrated in Eq. (14), with $\left(\sum_{i l} \alpha_{i l}^{(j)} a_{i} a_{l}\right)$ appearing on the production side and $\left(\sum_{m n} \alpha_{m n}^{(f)} a_{m} a_{n}\right)$ appearing on the decay side. We absorb the width $\Gamma_{H}$ in Eq. (14) into 
the overall signal strength and parametrize the kinematic dependence in the signal probability density on the ratio of couplings through $\vec{f}_{j}$ in the following way.

In the case of $H V V$ anomalous couplings, we have either $L=5$ couplings in approach 1 or $L=4$ couplings in approach 2, which we can parametrize with four or three components of $\vec{f}_{j}$, defined above, and $f_{a 1}=\left(1-\left|f_{a 2}\right|-\right.$ $\left.\left|f_{a 3}\right|-\left|f_{\Lambda 1}\right|-\left|f_{\Lambda 1}^{Z_{\gamma}}\right|\right)$. Let us denote these as $f_{l}$ with $l=1$, $2,3,4,5$. For the $g g H$ and $t \bar{t} H$ processes, when we consider anomalous couplings on the production side, we have only two parameters $f_{l}=\left(1-\left|f_{a 3}^{g g H}\right|\right)$ and $f_{a 3}^{g g H}$, or $\left(1-\left|f_{C P}^{H t t}\right|\right)$ and $f_{C P}^{H t t}$, and $L=2$. When developing the expression in Eq. (14), one gets a polynomial in the couplings $a_{l} \propto \sqrt{\left|f_{l}\right|} \operatorname{sign}\left(f_{l}\right)$ following Eq. (19), which is either quartic $(C=4)$, in the case of the EW processes, or quadratic $(C=2)$, when the couplings appear only on the decay or production side. Parametrization of the anomalous coupling dependence of the $\mathcal{P}_{j k}^{\text {sig }}$ probability density in Eq. (22) is different in these two cases.

This leads to the following general expression for the probability density of the EW processes with $C=4$

$$
\begin{aligned}
\mathcal{P}_{j k}^{\mathrm{sig}}\left(\vec{x} ; \vec{\xi}_{j k}, \vec{f}_{j}\right) \propto & \sum_{l \leq m \leq n \leq p=1}^{L} \mathcal{P}_{j k, l m n p}^{\mathrm{sig}}\left(\vec{x} ; \vec{\xi}_{j k}\right) \\
& \times \sqrt{\left|f_{l} f_{m} f_{n} f_{p}\right|} \operatorname{sign}\left(f_{l} f_{m} f_{n} f_{p}\right) .
\end{aligned}
$$

The following general expression applies to the probability density of the processes with $C=2$

$$
\mathcal{P}_{j k}^{\mathrm{sig}}\left(\vec{x} ; \vec{\xi}_{j k}, \vec{f}_{j}\right) \propto \sum_{l \leq m=1}^{L} \mathcal{P}_{j k, l m}^{\mathrm{sig}}\left(\vec{x} ; \vec{\xi}_{j k}\right) \sqrt{\left|f_{l} f_{m}\right|} \operatorname{sign}\left(f_{l} f_{m}\right)
$$

In both Eqs. (23) and (24), only the kinematic dependence on $\vec{f}_{j}$ is expressed, while the overall normalization can be absorbed into $\mu_{j}$ or accounted for as part of the cross section measurement.

In the general case, there are $(C+L-1) ! /(C !(L-1) !)$ terms in either Eqs. (23) or (24). This leads to 70 terms in Eq. (23) when we measure four anomalous $H V V$ couplings in production and decay $(L=5, C=4), 15$ terms in Eq. (24) when we measure four anomalous $H V V$ couplings in decay only ( $L=5, C=2)$, and three terms in Eq. (24) when we measure $\mathrm{Hgg}$ or $\mathrm{Htt}$ couplings in production only $(L=2, C=2)$. When both $H V V$ (in decay) and $H g g$ or $H t t$ (in production) couplings are measured at the same time in a given process, the number of terms is multiplied $(15 \times 3=45)$, since the two probabilities factorize.

The $\mathcal{P}_{j k, l m(n p)}^{\text {sig }}$ templates are extracted from simulation, discussed in Sec. III C, typically using from 3 to 12 samples generated with various $\vec{f}_{j}$ values chosen to map different points of phase-space well and reweighted with the MELA package to cover all possibilities with $(C+L-1)$ !/ $(C !(L-1) !)$ combinations of couplings. In parametrizing the signal templates $\mathcal{P}_{j k, l m(n p)}^{\text {sig }}$, it is important to ensure that the expected number of events in every bin of the probability densities, defined in Eqs. (23) and (24), remains nonnegative at all possible values of $\vec{f}_{j}$, because a negative yield would cause the likelihood function used for the final fit to become ill defined.

To detect a negative event yield, we first minimize Eqs. (23) or (24), which are polynomials in $\sqrt{\left|f_{l}\right|}$, by finding where the gradient is zero. For Eq. (24), which is quadratic, this is a simple problem in linear algebra. Equation (23) is quartic, so its gradient is a system of cubic equations, which cannot be solved exactly for $L>1$. We use the HOM4PS program [105-107] to numerically solve the system of equations. If we find the minimum to be negative, we adjust the estimated $\mathcal{P}_{j k, \text { smn }}^{\text {sig }}$ until the yield is always positive. This adjustment is made using the statistical uncertainty on $\mathcal{P}_{j k, l m n p}^{\text {sig }}$ through a cutting planes algorithm [108] implemented using the GUROBI program [109]. In all cases, it is found that only small modifications to the initial estimates of $\mathcal{P}_{j k, l m n p}^{\text {sig }}$ are needed.

The parametrization in Eqs. (23) and (24) is written in the self-consistent full-amplitude approach. In the EFT interpretation of the amplitude fit, the series in powers of $\left|f_{a i}\right|^{1 / 2}$ corresponds to terms of different dimension, as discussed in Sec. II C. For example, in Eq. (23) the term with $f_{a 1}^{2}$ corresponds to the SM-like contribution with dimension-4 operators, while $f_{a 1}^{3 / 2}\left|f_{a i}\right|^{1 / 2}$ corresponds to interference between the SM amplitude and the dimension6 contributions in the EFT expansion. Assuming that $f_{a 1} \sim 1$ and all $f_{a i}$ are small, all other terms could in principle be neglected as a test of EFT validity. In practice, however, neglecting those terms can easily lead to a negative probability in certain points in the phase space of observables, which invalidates the maximum likelihood fit. This does not necessarily mean that the EFT approach is not valid. This happens because the sizable interference terms can lead to a negative partial sum in Eq. (23), especially in the optimized multidimensional space of observables that are sensitive to such interference effects. Therefore, in practice, the fits presented in Sec. VI do not allow one to place constraints without the full series shown in Eqs. (23) and (24).

\section{Likelihood fit}

The final constraints on parameters $\mu_{j}$ and $\vec{f}_{j}$ are placed using the profile likelihood method implemented in the RooFit toolkit [110] within the ROOT [111] framework. The extended likelihood function is constructed using 
the probability densities in Eq. (22), with each event characterized by the discrete category $k$ and observables $\vec{x}$. The likelihood $\mathcal{L}$ is maximized with respect to the nuisance parameters $\vec{\xi}_{j k}$ describing the systematic uncertainties discussed below, and $\mu_{j}$ and $\vec{f}_{j}$ parameters of interest. The allowed 68 and $95 \% \mathrm{CL}$ intervals are defined using the profile likelihood function, $-2 \Delta \ln \mathcal{L}=1.00$ and 3.84, for which exact coverage is expected in the asymptotic limit [112].

The reinterpretation of the primary $\mu_{j}$ and $\vec{f}_{j}$ results in terms of couplings is performed with the help of Eq. (14) to relate signal strength $\mu_{j}$ to couplings and Eqs. (16)-(18) to relate $\vec{f}_{j}$ to coupling ratios. In this way the couplings directly enter the parametrization in Eq. (22). However, without further constraints on the $H$ boson width, such a fit would not provide useful constraints on the coupling size. Therefore, in the total width $\Gamma_{H}$ parametrization in Eq. (14), we assume that there are no unobserved or undetected $H$ boson decays. We express the width $\Gamma_{H}$ as the sum of partial decay widths of nine $H$ boson decay modes dominant in the SM, $H \rightarrow b \bar{b}$ being the largest and $H \rightarrow \mu^{+} \mu^{-}$being the smallest. Each partial decay width is scaled as a function of anomalous couplings following the parametrization in Ref. [33].

\section{Systematic uncertainties}

Several systematic uncertainties are considered in the set of constrained parameters $\vec{\xi}_{j k}$. The relative expected yields in different categories and the template shapes describing probability distributions in Eq. (22) are varied within either theoretical or experimental uncertainties. All results reported at 68 and 95\% CL are dominated by statistical uncertainties. All systematic uncertainties are treated as correlated between different time periods, except for the jetrelated uncertainties, which originate from statistically independent sources, and luminosity uncertainties, which are partially correlated [113-115].

The theoretical uncertainties considered are PDF parametrization, factorization, and renormalization scales, the hadronization scale used in PYTHIA, and the underlying event variations. The underlying event modeling uncertainty is determined by varying initial- and final-state radiation scales between 0.25 and 4 times their nominal value. The effects of the modeling of hadronization are determined by simulating additional events with the variation of the nominal PYTHIA tune described in Sec. III. Experimental uncertainties involve jet energy calibration and $b$-quark-tagging efficiency uncertainties, which are only relevant when production categories are considered, and lepton efficiency and momentum uncertainties, which are similar for the different processes and categories. In the estimation of the $Z+X$ background, the flavor composition of QCD-evolved jets misidentified as leptons may be different in the $Z+1 \ell$ and $Z+2 \ell$ control regions, and together with the statistical uncertainty in the $Z+2 \ell$ region, this uncertainty accounts for about a $\pm 30 \%$ variation in the $Z+X$ background.

The normalization of the background processes derived from the MC simulation is affected by a $1.8 \%$ uncertainty in the integrated luminosity. However, all results are found to be insensitive to luminosity or theoretical constraints on the nonresonant $Z Z / Z \gamma^{*} \rightarrow 4 \ell$ background. Even though the theoretical cross section is one of the constraints in the fit, the wide sideband included in the range $105<m_{4 \ell}<$ $140 \mathrm{GeV}$ constrains this nearly flat background from the data, with nearly identical results when the theoretical constraints are removed. The main distinguishing feature of this background is the dominant contribution of the $Z \gamma^{*}$ intermediate state, which allows effective separation of the already small background from signal using kinematic information.

\section{RESULTS}

The signal strength $\mu_{j}$ and the set of parameters $\vec{f}_{j}$ describing the tensor structure of interactions are constrained in each production process and decay $H \rightarrow$ $Z Z / Z \gamma^{*} / \gamma^{*} \gamma^{*} \rightarrow 4 \ell$. In the following, we describe the measurement of $f_{a 3}^{g g H}$ in the $g g H$ process, $f_{C P}^{H t t}$ in the $t \bar{t} H$ and $t H$ processes, and the combination of the two where the top quark contributes to the gluon fusion loop. We then report measurements of $\left(f_{a 2}, f_{a 3}, f_{\Lambda 1}, f_{\Lambda 1}^{Z_{\gamma}}\right)$ in the VBF and $V H$ processes along with the $H \rightarrow 4 \ell$ decay in all production processes, following approach 1 with the coupling relationship $a_{i}^{W W}=a_{i}^{Z Z}$. We also report measurements of $\left(f_{a 2}, f_{a 3}, f_{\Lambda 1}\right)$ following approach 2 within SMEFT, discussed in Sec. II. These results are interpreted in terms of constraints on the $H \mathrm{ff}, \mathrm{Hgg}$, and $H V V$ operators. While all operators could potentially be constrained in a joint analysis of all $H$ boson decay modes, in this paper we analyze only the $H \rightarrow 4 \ell$ decay mode, and perform a combination with the $t H$ and $t \bar{t} H$ processes in the $H \rightarrow \gamma \gamma$ decay mode. Therefore, for the purpose of illustration, we make further assumptions on how certain couplings, to which this analysis is not sensitive, are related. For example, we must make certain assumptions about the relationship between the $H b b, H c c, H \tau \tau$, and $H \mu \mu$ couplings and other couplings. These assumptions are discussed in each of the applications presented below.

\section{A. Constraints on $\mathbf{H g g}$ couplings}

The measurement of anomalous couplings of the $H$ boson to gluons is presented in Fig. 14 and Table V. Since the direct couplings of the $H$ boson to SM fermions in the gluon fusion loop and to potentially new particles appearing in the loop can not be resolved using this measurement alone, both effects are characterized with two parameters, $f_{a 3}^{g g H}$ and $\mu_{g g H}$. The signal strength $\mu_{g g H}$, which is the ratio 

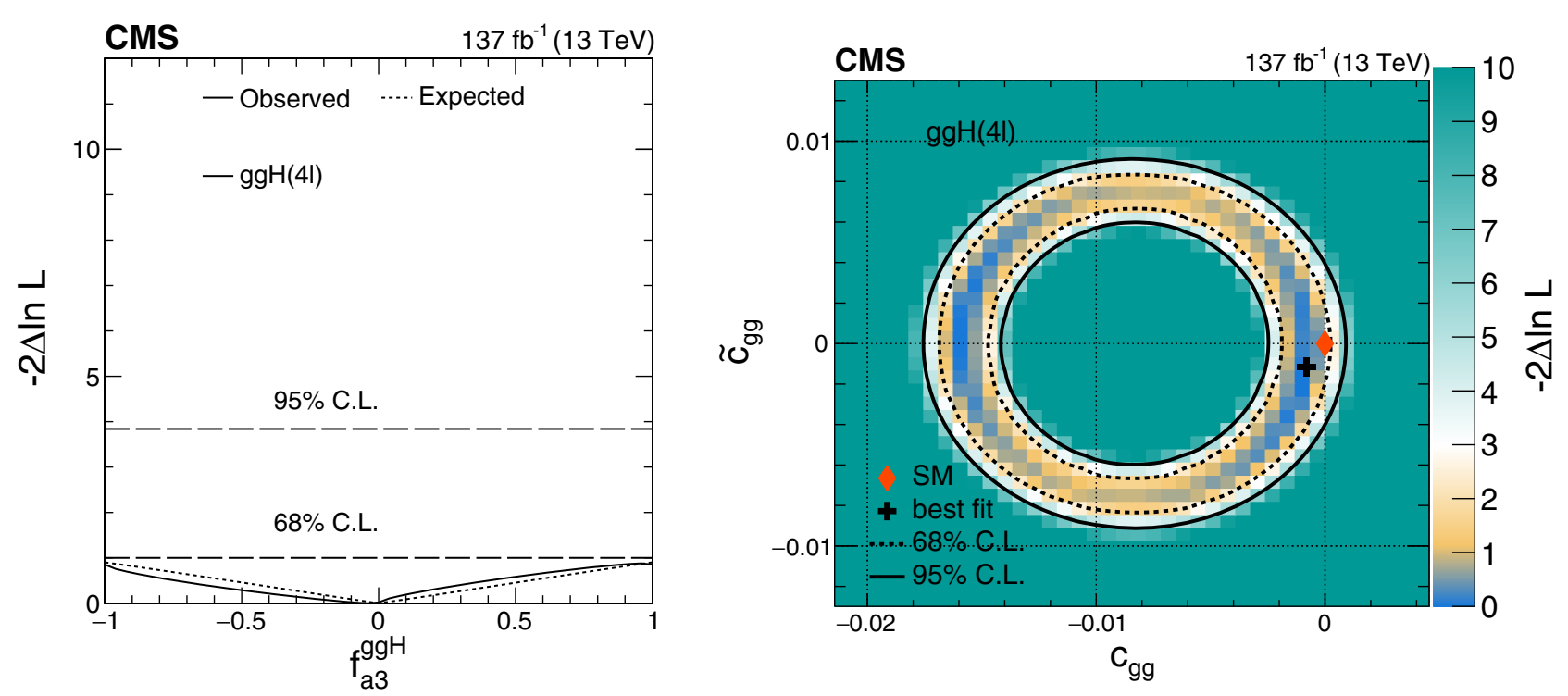

FIG. 14. Constraints on the anomalous $H$ boson couplings to gluons in the $g g H$ process using the $H \rightarrow 4 \ell$ decay. Left: observed (solid) and expected (dashed) likelihood scans of the $C P$-sensitive parameter $f_{a 3}^{g g H}$. The dashed horizontal lines show 68 and $95 \% \mathrm{CL}$ Right: observed confidence level intervals on the $c_{g g}$ and $\tilde{c}_{g g}$ couplings reinterpreted from the $f_{a 3}^{g g H}$ and $\mu_{g g H}$ measurement with $f_{a 3}$ and $\mu_{V}$ profiled, and with $\kappa_{t}=\kappa_{b}=1$. The dashed and solid lines show the 68 and 95\% CL exclusion regions in two dimensions, respectively.

of the measured cross section of the gluon fusion process to that expected in the SM, is profiled when the $f_{a 3}^{g g H}$ results are reported. The measurement of the $f_{a 3}^{g g H}$ is consistent with zero, as expected in the SM. This can be clearly seen from the $\mathcal{D}_{C P}^{g g H}$ and $\mathcal{D}_{0-}^{g g H}$ distributions in Fig. 10. The measured value of $\mu_{g g H}=0.86_{-0.11}^{+0.13}$ is consistent with that reported in Ref. [78] without the fit for the $C P$ structure of interactions. The values of $\mu_{g g H}$ and $f_{a 3}^{g g H}$ are uncorrelated. The signal strength of the VBF and $V H$ processes $\mu_{V}$ and their $C P$ properties $f_{a 3}$ are also profiled when this measurement is performed. This measurement is also performed simultaneously in a fit with the $t \bar{t} H$ process with the $\mu_{\bar{t} \bar{H}}$ and $f_{C P}^{H t t}$ parameters unconstrained, as discussed below. The $t H$ process is always included with the $t \bar{t} H$ process with its signal strength expressed through the $\mu_{\bar{t} \bar{H} H}, \mu_{V}$, and $f_{C P}^{H t t}$ parameters.

The parameters $f_{a 3}^{g g H}$ and $\mu_{g g H}$ are equivalent to the measurement of the $C P$-even and $C P$-odd couplings on the production side, while the $H V V$ couplings on the decay side are constrained from the simultaneous measurement of the VBF and $V H$ processes with $f_{a 3}$ and $\mu_{V}$ profiled. The $c_{g g}$ and $\tilde{c}_{g g}$ couplings, introduced in Eqs. (12) and (13), can be extracted from the above measurements. We follow the parametrization o $1 \mathrm{f}$ the cross section and the total width from Ref. [33], where $c_{g g}, \tilde{c}_{g g}, \kappa_{t}, \tilde{\kappa}_{t}, \kappa_{b}$, and $\tilde{\kappa}_{b}$ contribute. Since it is not possible to disentangle all these couplings in a single process, we fix $\kappa_{t}=\kappa_{b}=1$ and $\tilde{\kappa}_{t}=\tilde{\kappa}_{b}=0$ to the

TABLE V. Constraints on the $f_{a 3}^{g g H}$ and $f_{C P}^{H t t}$ parameters with the best fit values and allowed 68\% CL (quoted uncertainties) and $95 \%$ CL (within square brackets) intervals, limited to the physical range of $[-1,1]$. The $f_{C P}^{H t t}$ constraints obtained in this work are combined with those in the $H \rightarrow \gamma \gamma$ channel [26]. The interpretation of the $f_{a 3}^{g g H}$ result under the assumption of the top quark dominance in the gluon fusion loop are presented in terms of the $f_{C P}^{H t t}$ parameter, where either $g g H$ or its combination with $t H$ and $t \bar{t} H$ results are shown.

\begin{tabular}{|c|c|c|c|}
\hline Parameter & Scenario & Observed & Expected \\
\hline$f_{a 3}^{g g H}$ & $g g H(H \rightarrow 4 \ell)$ & $-0.04_{-0.96}^{+1.04}[-1,1]$ & $0 \pm 1[-1,1]$ \\
\hline$f_{C P}^{H t t}$ & $\begin{array}{l}t H \& t \bar{t} H(H \rightarrow 4 \ell) \\
t H \& t \bar{t} H(H \rightarrow \gamma \gamma)[26] \\
t H \& t \bar{t} H(H \rightarrow 4 \ell \& \gamma \gamma) \\
g g H(H \rightarrow 4 \ell) \\
g g H \& t H \& t \bar{t} H(H \rightarrow 4 \ell) \\
g g H \& t H \& t \bar{t} H(H \rightarrow 4 \ell \& \gamma \gamma)\end{array}$ & $\begin{array}{c} \pm\left(0.88_{-1.88}^{+0.12}\right)[-1,1] \\
0.00 \pm 0.33[-0.67,0.67] \\
0.00 \pm 0.33[-0.67,0.67] \\
-0.01_{-0.99}^{+1.01}[-1,1] \\
-0.56_{-0.44}^{+1.56}[-1,1] \\
-0.04_{-0.36}^{+0.38}[-0.69,0.68]\end{array}$ & $\begin{array}{c}0 \pm 1[-1,1] \\
0.00 \pm 0.49[-0.82,0.82] \\
0.00 \pm 0.48[-0.81,0.81] \\
0 \pm 1[-1,1] \\
0.00 \pm 0.47[-1,1] \\
0.00 \pm 0.30[-0.70,0.70]\end{array}$ \\
\hline
\end{tabular}


SM expectation and leave $c_{g g}$ and $\tilde{c}_{g g}$, which describe possible BSM contribution in the loop, unconstrained. The small contribution of the $H \rightarrow \gamma \gamma$ and $Z \gamma$ decays to the total width is assumed to be SM-like.

The resulting constraints on $c_{g g}$ and $\tilde{c}_{g g}$ are shown in Fig. 14, right. The general features of these constraints are the following. The pure signal strength measurement $\mu_{g g H}$, available even without the fit for $f_{a 3}^{g g H}$, provides a constraint in the form of a ring on a two-parameter plane in Fig. 14, right. The measurement of $f_{a 3}^{g g H}$ resolves the areas within this ring. Since the sensitivity of the $f_{a 3}^{g g H}$ measurement is currently just under $68 \% \mathrm{CL}$, this resolution is not strong. The $H$ boson width dependence on $c_{g g}$ and $\tilde{c}_{g g}$ is relatively weak and does not alter this logic considerably. The results are consistent with the SM expectation.

As mentioned earlier, it is not possible to resolve the loop contributions from the SM or BSM particles in this measurement. Therefore, the deviations of the SM-like Yukawa couplings $\kappa_{t}$ and $\kappa_{b}$ from unit values are absorbed into the effective $c_{g g}$ measurement, and the $C P$-odd Yukawa couplings $\tilde{\kappa}_{t}$ and $\tilde{\kappa}_{b}$ are absorbed into the effective $\tilde{c}_{g g}$ measurement, together with possible contributions from BSM particles. However, reinterpretation of these results is possible in terms of the independent Yukawa couplings and effective pointlike gluon couplings in combination with the $t \bar{t} H$ and $t H$ modes, as discussed below.

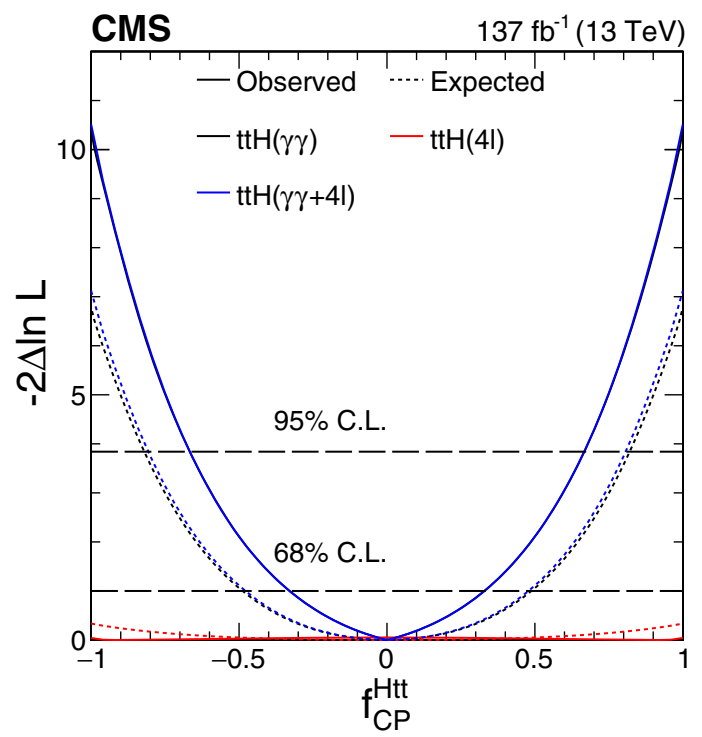

\section{B. Constraints on $\mathrm{Htt}$ couplings}

The measurement of anomalous couplings of the $H$ boson to top quarks is presented in Fig. 15 and Table V. First, the measurements of $f_{C P}^{H t t}$ from the $t \bar{t} H$ and $t H$ processes only are reported. The signal strength $\mu_{\bar{t} \bar{t} H}$, which is the ratio of the measured cross section of the $\bar{t} H$ process to that expected in the $\mathrm{SM}$, is profiled when the $f_{C P}^{H t t}$ results are reported. The measured value of $\mu_{t \bar{t} H}=0.17_{-0.17}^{+0.70}$ is consistent with that reported in Ref. [78] without the fit for the $C P$ structure of interactions. In both cases we observe downward fluctuations in the signal yield compared to expectation, but these fluctuations are not statistically significant. There is no significant linear correlation between $\mu_{t \bar{t} H}$ and $f_{C P}^{H t t}$. The signal strength of the VBF and $V H$ processes $\mu_{V}, g g H$ process $\mu_{g g H}$, and their $C P$ properties $f_{a 3}$ and $f_{a 3}^{g g H}$ are also profiled when this measurement is performed. This analysis of the $t \bar{t} H$ and $t H$ processes is not sensitive to the sign of $f_{C P}^{H t t}$. However, for later combination with the $g g H$ measurement, presented above, under the assumption of the top quark dominance in the gluon fusion loop, symmetric constraints on $f_{C P}^{H t t}$ are reported.

The observed best fit $f_{C P}^{H t t}$ value gives preference to the $C P$-odd Yukawa coupling. This comes from the negative value of the $\mathcal{D}_{0-}^{\bar{t} t H}$ discriminant for the one observed signallike event in Fig. 9. However, this result is statistically consistent with the pure $C P$-even Yukawa coupling expected in the SM. With just about two signal $t \bar{t} H$ events

CMS

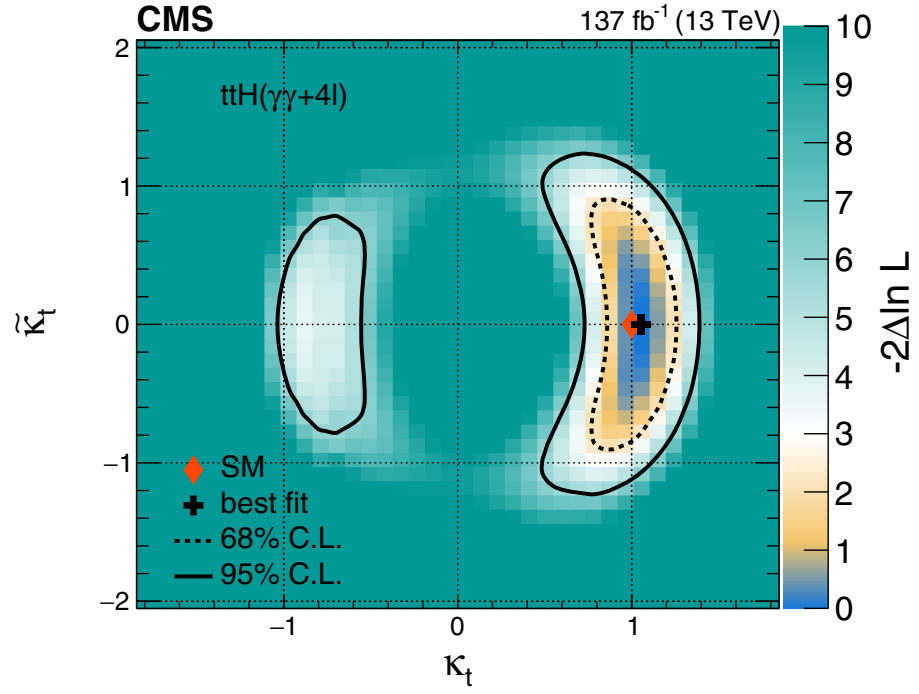

FIG. 15. Constraints on the anomalous $H$ boson couplings to top quarks in the $t \bar{t} H$ and $t H$ processes using the $H \rightarrow \gamma \gamma$ [26] and $H \rightarrow 4 \ell$ decays. Left: observed (solid) and expected (dashed) likelihood scans of $f_{C P}^{H t t}$ in the $t \bar{t} H$ and $t H$ processes in the $H \rightarrow 4 \ell$ (red), $\gamma \gamma$ (black), and combined (blue) channels, where the combination is done without relating the signal strengths in the two processes. The dashed horizontal lines show 68 and 95\% CL Right: observed confidence level intervals on the $\kappa_{t}$ and $\tilde{\kappa}_{t}$ couplings reinterpreted from the $f_{C P}^{H t t}, \mu_{t \bar{t} H}$, and $\mu_{V}$ measurements in the combined fit of the $H \rightarrow 4 \ell$ and $\gamma \gamma$ channels, with the signal strengths in the two channels related through the couplings as discussed in text. The dashed and solid lines show the 68 and 95\% CL exclusion regions in two dimensions, respectively. 
and many fewer $t H$ events expected to appear in the fit in the $H \rightarrow 4 \ell$ channel under the assumption of the SM cross section, according to Table II, the expected confidence sensitivity on the $f_{C P}^{H t t}$ constraints is low. Nonetheless, the high signal purity in the $H \rightarrow 4 \ell$ channel implies that every observed event candidate carries a large statistical weight. The importance of including the $C P$ measurements in the $t \bar{t} H$ and $t H$ production modes also becomes evident when combination with $g g H$ is performed. There is a significant gain in such a combination beyond a simple addition of independent measurements, as discussed in Sec. VIC.

The CMS experiment recently reported the measurement of the $f_{C P}^{H t t}$ parameter in the $t \bar{t} H$ and $t H$ production processes with the decay $H \rightarrow \gamma \gamma$ [26] (shown also in Table V). In that measurement, the signal strength $\mu_{t \bar{t} H}^{\gamma \gamma}$ parameter is profiled, while the signal strengths in other production processes are fixed to the SM expectation. However, there is a very weak correlation of the measurement in the $t \bar{t} H$ and $t H$ processes with parameters in the other production mechanisms. Therefore, we proceed with a combination of the $f_{C P}^{H t t}$ measurements in the $H \rightarrow 4 \ell$ and $\gamma \gamma$ channels, where we correlate their common systematic uncertainties, but not the signal strengths of the processes. In particular, we do not relate the $\mu_{t \bar{t} H}$ and $\mu_{t \bar{H} H}^{\gamma \gamma}$ signal strengths because they could be affected differently by the particles appearing in the loops responsible for the $H \rightarrow \gamma \gamma$ decay. The results of this combination are presented in Fig. 15 and Table V. The measured signal strength in the $H \rightarrow 4 \ell$ channel is $\mu_{\bar{t} \bar{H} H}=0.04_{-0.04}^{+0.76}$, uncorrelated with $f_{C P}^{H t t}$, while $\mu_{t \bar{t} H}^{\gamma \gamma}=$ $1.23_{-0.24}^{+0.33}$ and the correlation with $f_{C P}^{H t t}$ is +0.20 . The pure pseudoscalar hypothesis of the $H$ boson corresponding to $f_{C P}^{H t t}=1$ in the case of the $C P$-odd Yukawa interaction is excluded at 3.2 standard deviations, while the expected exclusion is 2.7 standard deviations. Below, we also present an interpretation of these results where the signal strengths in the two $H$ boson decay channels are related through the couplings.

In the above measurements, the $f_{C P}^{H t t}$ parameter has the same meaning in both the $H \rightarrow 4 \ell$ and $\gamma \gamma$ channels. In order to make an EFT coupling interpretation of the results, we have to make a further assumption that no BSM particles contribute to the loop in the $H \rightarrow \gamma \gamma$ decay. Without this or a similar assumption, the signal strength in the $H \rightarrow \gamma \gamma$ decay cannot be interpreted without ambiguity. We further reparametrize the cross section following Ref. [33] with the couplings $\kappa_{t}$ and $\tilde{\kappa}_{t}$, and fix $\kappa_{b}=1$ and $\tilde{\kappa}_{b}=0$. The bottom quark coupling makes a very small contribution to the loop in the $H \rightarrow \gamma \gamma$ decay, but it makes a large contribution to the total decay width, where we assume that there are no unobserved or undetected $H$ boson decays. In order to simplify the fit, we do not allow anomalous $H V V$ couplings, and the measurement of the signal strength $\mu_{V}$ constrains the contribution of the $a_{1}$ coupling in the loop. The $f_{a 3}^{g g H}$ and $\mu_{g g H}$ parameters are profiled in this fit.

The observed confidence level intervals on the $\kappa_{t}$ and $\tilde{\kappa}_{t}$ couplings from the combined fit of the $H \rightarrow 4 \ell$ and $\gamma \gamma$ channels are shown in Fig. 15. There is no linear correlation between the values of $\kappa_{t}$ and $\tilde{\kappa}_{t}$. As was the case for the $\left(c_{g g}, \tilde{c}_{g g}\right)$ measurement in Fig. 14, the pure yield measurement in the $t \bar{t} H$ process would constrain a ring in the two-dimensional plane. However, the $C P$-sensitive measurement of $f_{C P}^{H t t}$ disfavors the values away from $\tilde{\kappa}_{t}=0$. Moreover, the sign ambiguity between the $\kappa_{t}$ and $-\kappa_{t}$ values cannot be resolved in the $t \bar{t} H$ channel alone. With the inclusion of the $t H$ process, the negative values of $\kappa_{t}$ are disfavored because strong constructive interference between the amplitudes induced by the $\mathrm{HVV}$ and $\mathrm{Htt}$ couplings would result in enhanced $t H$ yield, inconsistent with the data. Therefore, the sign of $\kappa_{t}$ is defined in reference to the tree-level $H V V$ coupling $a_{1}$. But, the sign ambiguity between the $\tilde{\kappa}_{t}$ and $-\tilde{\kappa}_{t}$ values cannot be resolved in this fit, unless information from the other channels is incorporated, such as information from the gluon fusion loop discussed below.

\section{Constraints on $\mathrm{Htt}$ and $\mathrm{Hgg}$ couplings in combination}

First, we consider the $g g H$ process under the assumption of top quark dominance in the gluon fusion loop. The measurement of anomalous couplings of the $H$ boson to top quarks for this case is presented in Fig. 16 and Table V. Similar to the case of the $H \rightarrow \gamma \gamma$ loop discussed above, the cross section of the $g g H$ process, normalized to the SM expectation, is parametrized following Ref. [33] to account for $\mathrm{CP}$-odd Yukawa couplings as follows:

$$
\frac{\sigma(g g H)}{\sigma_{\mathrm{SM}}}=\kappa_{\mathrm{f}}^{2}+2.38 \tilde{\kappa}_{\mathrm{f}}^{2},
$$

where we set $\kappa_{\mathrm{f}}=\kappa_{t}=\kappa_{b}$ and $\tilde{\kappa}_{\mathrm{f}}=\tilde{\kappa}_{t}=\tilde{\kappa}_{b}$. Equation (25) sets the relationship between $f_{C P}^{H t t}$ and $f_{a 3}^{g g H}$, reported in Fig. 14 and Table V, according to Eq. (17).

Constraints on $f_{C P}^{H t t}$ are also shown for the combination of the $\bar{t} H, t H$, and $g g H$ processes with $H \rightarrow 4 \ell$ in Fig. 16 and Table V. The combination of the $H \rightarrow 4 \ell$ and $\gamma \gamma$ channels with $t \bar{t} H, t H$, and $g g H$ processes proceeds in a similar manner and is also shown in Fig. 16 and Table V. In this case, we do not allow anomalous $H V V$ couplings, and the measurement of the signal strength $\mu_{V}$ constrains the contribution of the $a_{1}$ coupling in the $H \rightarrow \gamma \gamma$ loop.

The gain in this combination of the $g g H$ and $t H$ and $t \bar{t} H$ processes is beyond the simple addition of the two constraints. While in the $g g H$ and $t \bar{t} H$ analyses the signal strengths of the two processes are independent, they could be related under the assumption of top quark dominance in the loop using Eq. (25). As discussed in Sec. II, $C P$-odd 


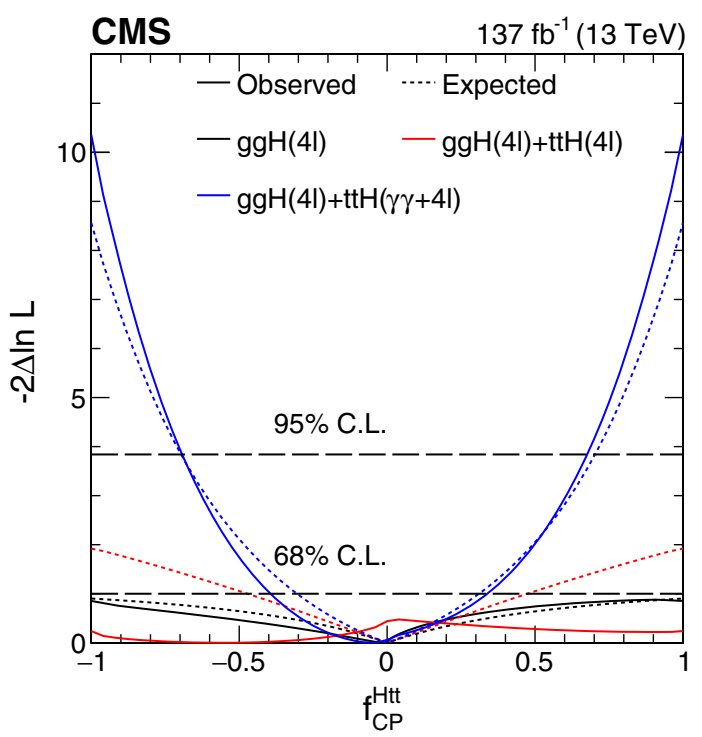

FIG. 16. Constraints on the anomalous $H$ boson couplings to top quarks in the $t \bar{t} H, t H$, and $g g H$ processes combined, assuming top quark dominance in the gluon fusion loop, using the $H \rightarrow 4 \ell$ and $\gamma \gamma$ decays. Observed (solid) and expected (dashed) likelihood scans of $f_{C P}^{H t t}$ are shown in the $g g H$ process with $H \rightarrow 4 \ell$ (black), $t \bar{t} H, t H$, and $g g H$ processes combined with $H \rightarrow 4 \ell$ (red), and in the $t \bar{t} H, t H$, and $g g H$ processes with $H \rightarrow 4 \ell$ and the $t \bar{t} H$ and $t H$ processes with $\gamma \gamma$ combined (blue). Combination is done by relating the signal strengths in the three processes through the couplings in the loops in both production and decay, as discussed in the text. The dashed horizontal lines show 68 and 95\% CL exclusion.

coupling predicts rather different cross sections in the two processes: $\sigma\left(\tilde{\kappa}_{\mathrm{f}}=1\right) / \sigma\left(\kappa_{\mathrm{f}}=1\right)$ is 2.38 in the gluon fusion process dominated by the top quark loop and 0.391 in the $\bar{t} \bar{t} H$ process. This means that the ratio differs by a factor of 6.09 for $f_{C P}^{H t t}=1$ when compared to SM $\left(f_{C P}^{H t t}=0\right)$. This correlation enhances the sensitivity in the $f_{C P}^{H t t}$ measurement. For example, the combined sensitivity from $t H$ and $\bar{t} \bar{t} H$ (with either $H \rightarrow 4 \ell$ alone or together with $H \rightarrow \gamma \gamma$ ), and $g g H$ is significantly improved compared to separate analyses, and the result is not just a simple addition of two independent results, as shown in Fig. 16 and Table V. This effect also enhances correlation between $f_{C P}^{H t t}$ and the yield parameters. In the full combination, the measured signal strength is $\mu_{t \bar{t} H}=0.70_{-0.25}^{+0.30}$ and the correlation with $f_{C P}^{H t t}$ is +0.96 .

Finally, we present the reinterpretation of the $f_{a 3}^{g g H}, f_{C P}^{H t t}$, and signal strength measurements in terms of constraints on $c_{g g}, \tilde{c}_{g g}, \kappa_{t}$, and $\tilde{\kappa}_{t}$. In this fit, it is assumed that $\kappa_{b}=\kappa_{c}=$ $\kappa_{\mu}=1$ and $\tilde{\kappa}_{b}=\tilde{\kappa}_{c}=\tilde{\kappa}_{\mu}=0$ in the fermion coupling contribution to the loops and in the decay width parametrization [33]. The gluon fusion loop is parametrized in terms of pointlike couplings $c_{g g}$ and $\tilde{c}_{g g}$, and the top and bottom quark contributions. These pointlike and top couplings can be resolved in combination with the $t \bar{t} H$ and $t H$ production processes. The $H \rightarrow \gamma \gamma$ loop is parametrized with the top and bottom quark and with the $W$ contributions. One cannot generally relate the pointlike couplings in this loop and in the gluon fusion loop, and they are assumed to be zero in $H \rightarrow \gamma \gamma$. The measurement of the signal strength $\mu_{V}$ constrains the contributions of the $a_{1}$ coupling, affecting the $W$ contribution to the $H \rightarrow \gamma \gamma$ loop and $t H$ process, and anomalous $H V V$ couplings are not allowed. By convention, the $a_{1}$ coupling is constrained to be positive, which sets the relative sign of $\kappa_{t}$. It is assumed that there are no unobserved or undetected $H$ boson decays.

The constraints are shown in Fig. 17, where the likelihood scans are plotted for the pairs of parameters: $\left(c_{g g}, \tilde{c}_{g g}\right),\left(\kappa_{t}, \tilde{\kappa}_{t}\right)$, and $\left(\kappa_{t}, c_{g g}\right)$, with the other two parameters either profiled or fixed to the SM expectation. On the likelihood scan of $\left(c_{g g}, \tilde{c}_{g g}\right)$ with the other parameters fixed, the appearance is generally similar to the scan shown in Fig. 14. This is because the addition of the $t \bar{t} H$ and $t H$ processes does not alter the results much with their couplings fixed to the SM. However, with the other parameters affecting the gluon fusion loop left floating, the contours are washed out, as one would expect with more degrees of freedom in a fit.

On the likelihood scan of $\left(\kappa_{t}, \tilde{\kappa}_{t}\right)$ with the other parameters profiled, one can observe the effects similar to that in Fig. 15. This is because the $g g H$ process does not bring additional constraints due to uncertainties with the pointlike interactions, but may introduce additional modifications to the fit parameters. However, with the pointlike interactions $c_{g g}$ and $\tilde{c}_{g g}$ set to zero, constraints on the $\kappa_{t}$ and $\tilde{\kappa}_{t}$ couplings appear tighter as a result of the combination of information from the $t \bar{t} H, t H$, and $g g H$ processes. In both cases, the general features remain similar to Fig. 15, such as pure yield measurements leading to constraints within a ring, $C P$-sensitive measurements resolving areas within this ring, and the $t H$ process leading to the exclusion of negative values of $\kappa_{t}$. However, in this case, ambiguity between the positive and negative values of $\tilde{\kappa}_{t}$ can be resolved with the inclusion of the $g g H$ process, where the $\mathcal{D}_{C P}^{g g H}$ discriminant carries information sensitive to the sign.

On the likelihood scan of $\left(\kappa_{t}, c_{g g}\right)$ with the other parameters fixed, we observe a resolved fourfold ambiguity of the best fit ranges. Within each range, there is a large correlation between the two parameters. This happens because the pointlike interaction $c_{g g}$ is equivalent to a BSM heavy quark $Q$ contribution to the loop. It is hard to distinguish between such a new heavy quark and the heavy top quark. The two amplitudes add constructively, leading to a large anticorrelation. The rate of the gluon fusion process would be roughly proportional to $\left(\kappa_{t}+\alpha \kappa_{Q}\right)^{2}$ with $\alpha \sim 1$. Given that the $t \bar{t} H$ rate constrains $\kappa_{t} \sim \pm 1$, the $g g H$ rate would constrain $\left(\kappa_{t}, \kappa_{Q}\right)$ to four discrete sets of values around $(+1,0),(+1,-2),(-1,0)$, and $(-1,+2)$. The presence of the $t H$ process shifts the preferred negative 

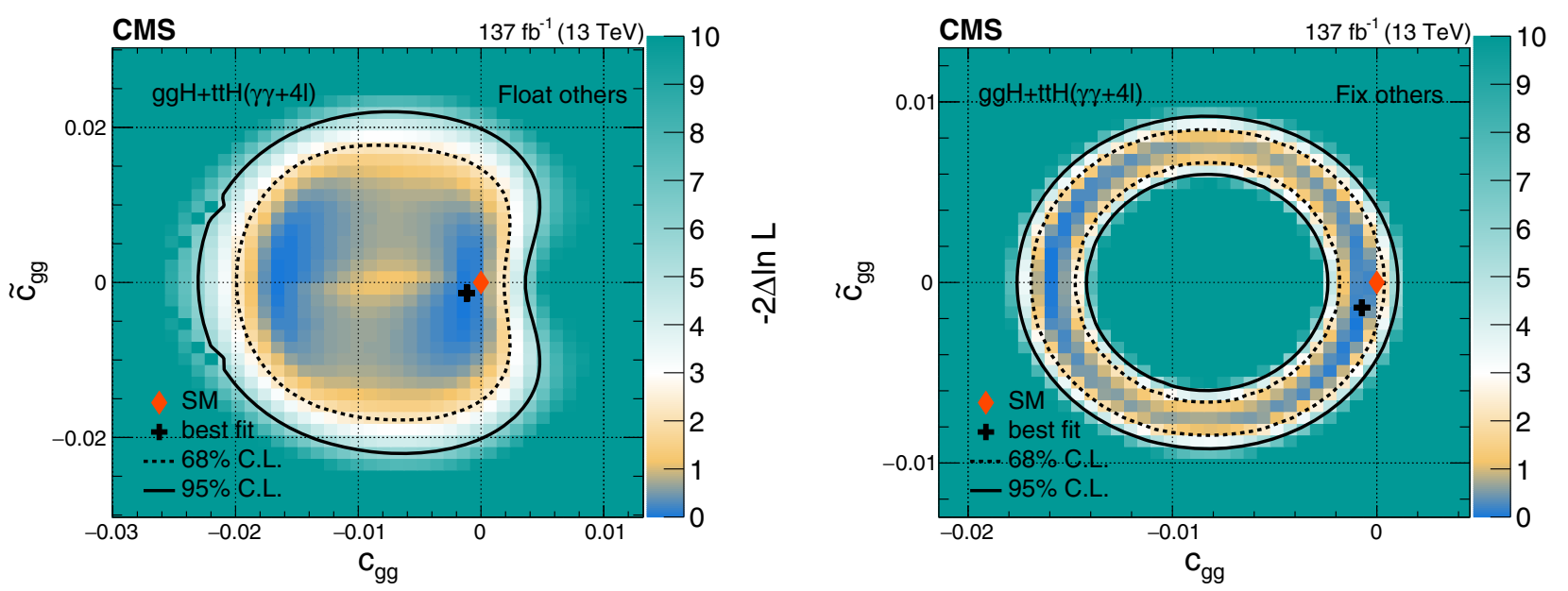

$\frac{\square}{\sqrt{~}}$
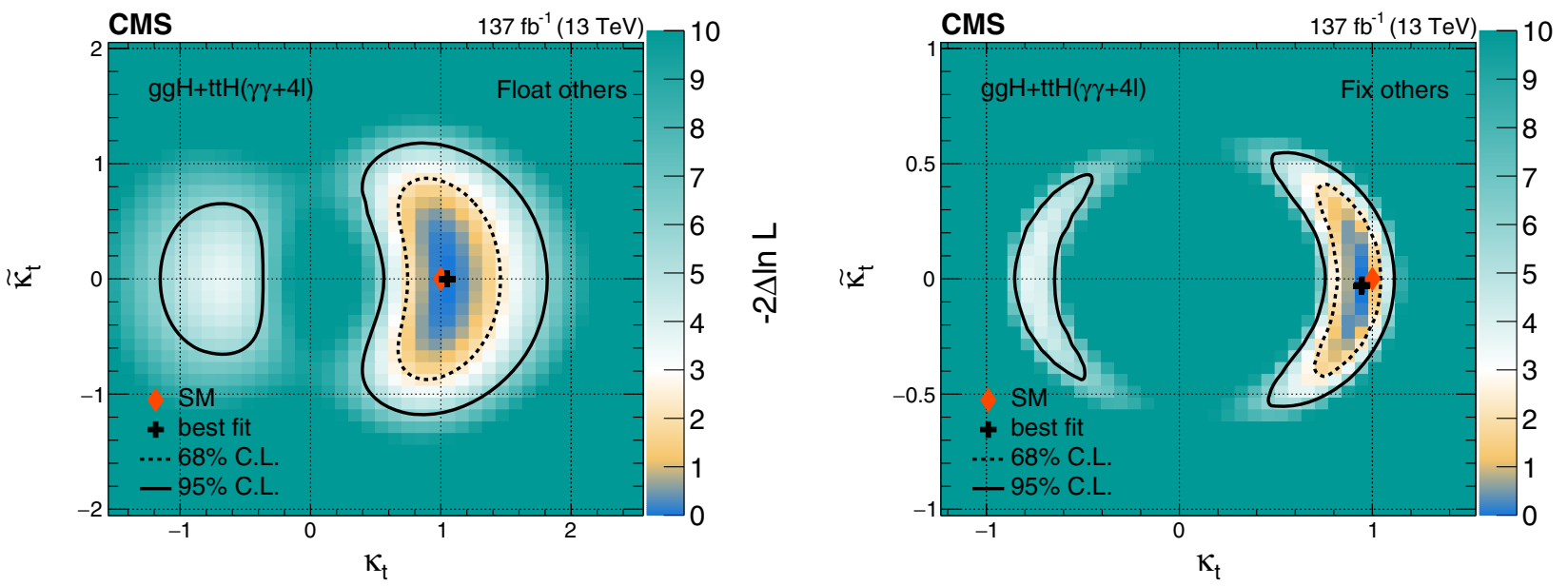

$\frac{1}{\sqrt{J}}$
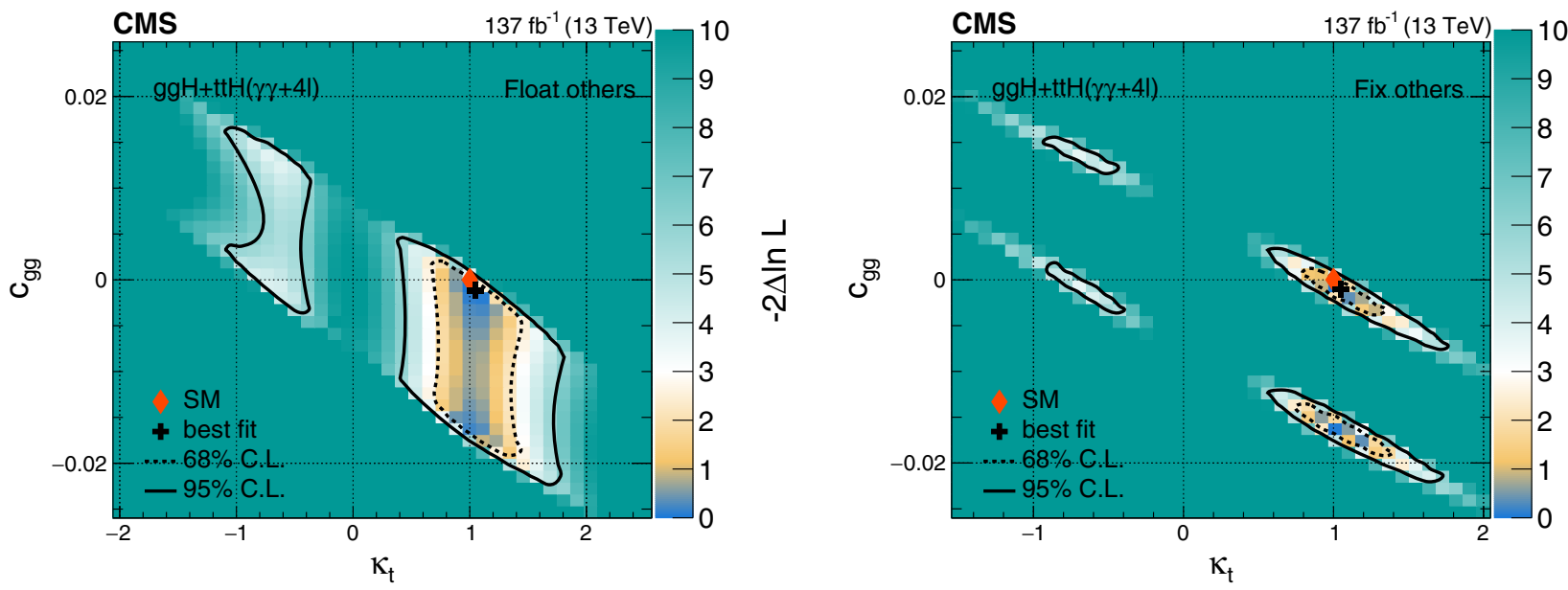

$\frac{1}{}$

FIG. 17. Constraints on the anomalous $H$ boson couplings $c_{g g}, \tilde{c}_{g g}, \kappa_{t}$, and $\tilde{\kappa}_{t}$ in the $\bar{t} H$, $t H$, and $g g H$ processes combined, using the $H \rightarrow 4 \ell$ and $\gamma \gamma$ decays. The constraints are shown for the pairs of parameters: $c_{g g}$ and $\tilde{c}_{g g}$ (upper), $\kappa_{t}$ and $\tilde{\kappa}_{t}$ (middle), $\kappa_{t}$ and $c_{g g}$ (lower), and with the other two parameters either profiled (left) or fixed to the SM expectation (right). The dashed and solid lines show the 68 and $95 \%$ CL exclusion regions in two dimensions, respectively.

$\kappa_{t}$ solutions away from -1 and makes it less likely than the +1 value for the reasons discussed above. However, the local minimum near $\kappa_{Q} \sim-2$, corresponding to $c_{g g} \sim-0.017$, cannot be excluded, even though the global minimum is at $c_{g g}=-0.001$, close to the null SM expectation. In the case with the other parameters profiled, the constraints on the $\left(\kappa_{t}, c_{g g}\right)$ plane get washed out further, as expected in a fit with more degrees of freedom. In this case, the $C P$-odd amplitudes can compensate for some effects of the $C P$-even ones. However, some sensitivity is 

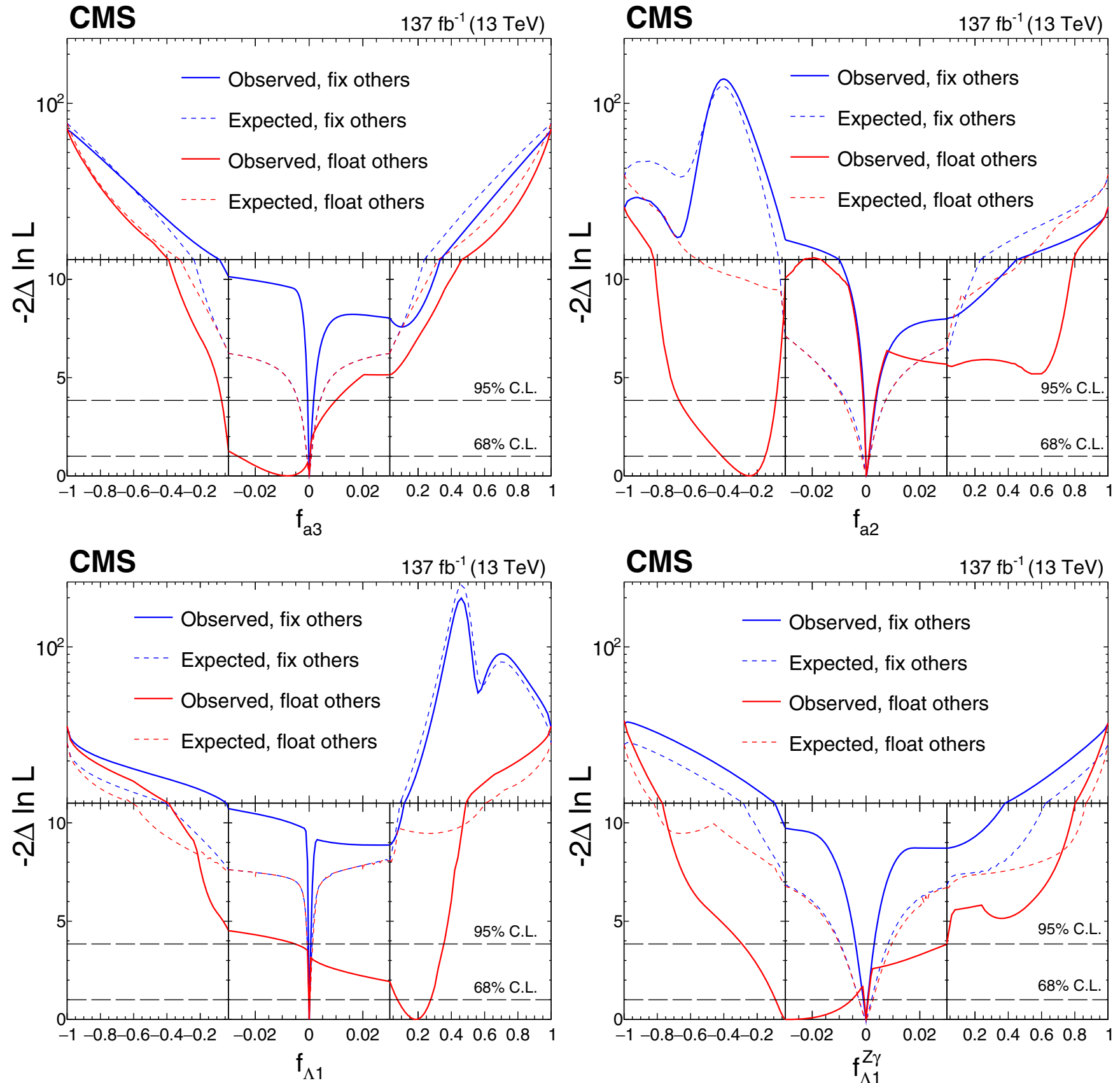

CMS $137 \mathrm{fb}^{-1}(13 \mathrm{TeV})$

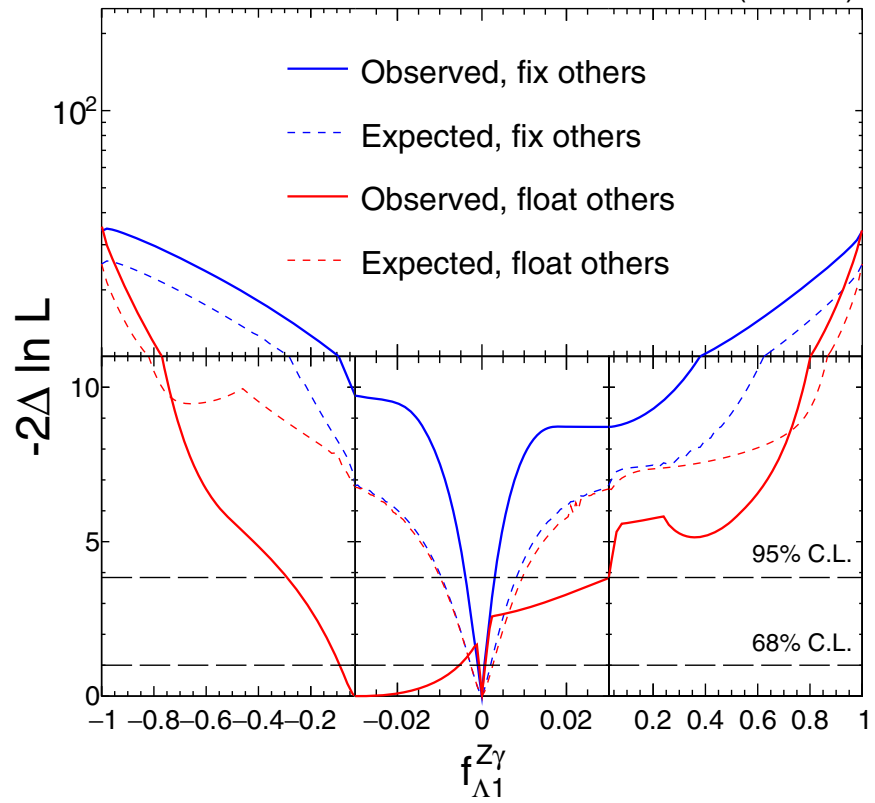

FIG. 18. Observed (solid) and expected (dashed) likelihood scans of $f_{a 3}$ (upper left), $f_{a 2}$ (upper right), $f_{\Lambda 1}$ (lower left), and $f_{\Lambda 1}^{Z \gamma}$ (lower right) in approach 1 with the coupling relationship $a_{i}^{W W}=a_{i}^{Z Z}$. The results are shown for each coupling fraction separately with the other three anomalous coupling fractions either set to zero or left unconstrained in the fit. In all cases, the signal strength parameters have been left unconstrained. The dashed horizontal lines show the 68 and 95\% CL regions. For better visibility of all features, the $x$ and $y$ axes are presented with variable scales. On the linear-scale $x$ axis, an enlargement is applied in the range -0.03 to 0.03 . The $y$ axis is shown in linear or logarithmic scale for values of $-2 \ln \mathcal{L}$ below or above 11 , respectively.

retained because $C P$-sensitive measurements constrain the relative contribution of $C P$-odd amplitudes.

\section{Constraints on $H V V$ couplings}

The measurement of anomalous couplings of the $H$ boson to EW vector bosons in approach 1 with the relationship $a_{i}^{W W}=a_{i}^{Z Z}$ is presented in Fig. 18 and Table VI. Figure 18 shows the observed and expected likelihood scans in the simultaneous measurement of $f_{a 3}$, $f_{a 2}, f_{\Lambda 1}$, and $f_{\Lambda 1}^{Z \gamma}$, where the $C P$-sensitive parameter $f_{a 3}^{g g H}$ and the signal strength parameters $\mu_{V}$ and $\mu_{g g H}$ are profiled, and where we relate $\mu_{\bar{t} \bar{H} H}$ and $f_{C P}^{H t t}$ to $\mu_{g g H}$ and $f_{a 3}^{g g H}$ 

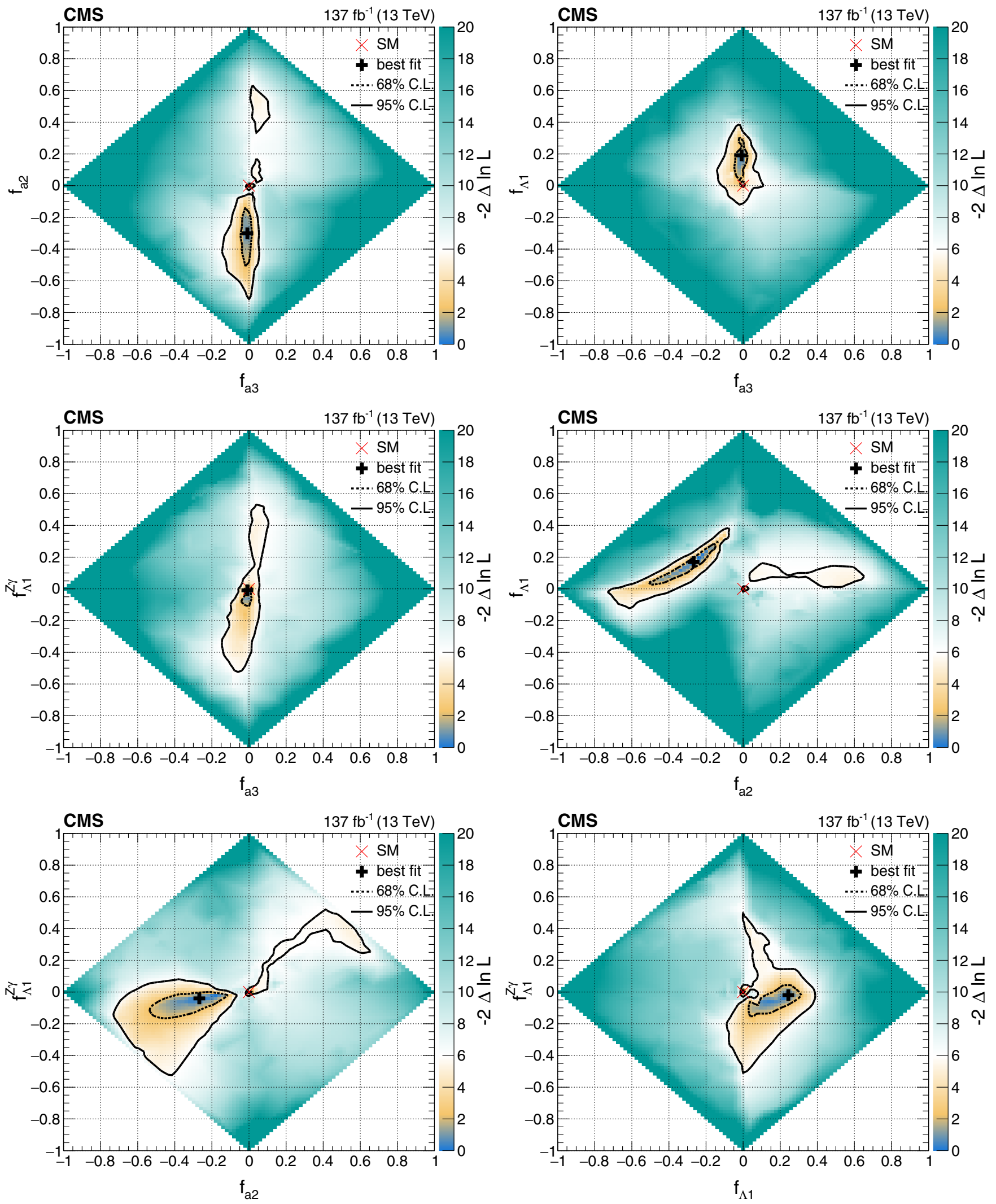

FIG. 19. Observed two-dimensional likelihood scans of the four coupling parameters $f_{a 3}, f_{a 2}, f_{\Lambda 1}$, and $f_{\Lambda 1}^{Z \gamma}$ in approach 1 with the coupling relationship $a_{i}^{W W}=a_{i}^{Z Z}$. In each case, the other two anomalous couplings along with the signal strength parameters have been left unconstrained. The 68 and $95 \%$ CL regions are presented as contours with dashed and solid black lines, respectively. The best fit values and the SM expectations are indicated by markers. 
TABLE VI. Summary of constraints on the anomalous $H V V$ coupling parameters with the best fit values and allowed 68 and $95 \%$ CL intervals. Three scenarios are shown for each parameter: with three other anomalous $H V V$ couplings set to zero (first), with three other anomalous $H V V$ couplings left unconstrained (second), in approach 1 with the relationship $a_{i}^{W W}=a_{i}^{Z Z}$ in both cases; and with two other anomalous $H V V$ couplings left unconstrained (third), in approach 2 within SMEFT with the symmetry relationship of couplings set in Eqs. (3)-(7). The $f_{\Lambda 1}^{Z \gamma}$ parameter is not independent in the latter scenario.

\begin{tabular}{|c|c|c|c|c|}
\hline Parameter & Scenario & & Observed & Expected \\
\hline$f_{a 3}$ & $\begin{array}{l}\text { Approach 1 } \\
f_{a 2}=f_{\Lambda 1}=f_{\Lambda 1}^{Z \gamma}=0 \\
\text { Approach 1 } \\
\text { float } f_{a 2}, f_{\Lambda 1}, f_{\Lambda 1}^{Z \gamma} \\
\text { Approach 2 } \\
\text { float } f_{a 2}, f_{\Lambda 1}\end{array}$ & $\begin{array}{l}\text { best fit } \\
68 \% \mathrm{CL} \\
95 \% \mathrm{CL} \\
\text { best fit } \\
68 \% \mathrm{CL} \\
95 \% \mathrm{CL} \\
\text { best fit } \\
68 \% \mathrm{CL} \\
95 \% \mathrm{CL}\end{array}$ & $\begin{array}{l}0.00004 \\
{[-0.00007,0.00044]} \\
{[-0.00055,0.00168]} \\
-0.00805 \\
{[-0.02656,0.00034]} \\
{[-0.07191,0.00990]} \\
0.00005 \\
{[-0.00010,0.00061]} \\
{[-0.00072,0.00218]}\end{array}$ & $\begin{array}{l}0.00000 \\
{[-0.00081,0.00081]} \\
{[-0.00412,0.00412]} \\
0.00000 \\
{[-0.00086,0.00086]} \\
{[-0.00423,0.00422]} \\
0.0000 \\
{[-0.0012,0.0012]} \\
{[-0.0057,0.0057]}\end{array}$ \\
\hline$f_{a 2}$ & $\begin{array}{l}\text { Approach 1 } \\
f_{a 3}=f_{\Lambda 1}=f_{\Lambda 1}^{Z \gamma}=0 \\
\text { Approach 1 } \\
\text { float } f_{a 3}, f_{\Lambda 1}, f_{\Lambda 1}^{Z \gamma} \\
\text { Approach 2 } \\
\text { float } f_{a 3}, f_{\Lambda 1}\end{array}$ & $\begin{array}{l}\text { best fit } \\
68 \% \mathrm{CL} \\
95 \% \mathrm{CL} \\
\text { best fit } \\
68 \% \mathrm{CL} \\
95 \% \mathrm{CL} \\
\text { best fit } \\
68 \% \mathrm{CL} \\
95 \% \mathrm{CL}\end{array}$ & $\begin{array}{l}0.00020 \\
{[-0.00010,0.00109]} \\
{[-0.00078,0.00368]} \\
-0.24679 \\
{[-0.41087,-0.15149] \cup[-0.00008,0.00065]} \\
{[-0.66842,-0.08754] \cup[-0.00091,0.00309]} \\
-0.00002 \\
{[-0.00178,0.00103]} \\
{[-0.00694,0.00536]}\end{array}$ & $\begin{array}{l}0.0000 \\
{[-0.0012,0.0014]} \\
{[-0.0075,0.0073]} \\
0.0000 \\
{[-0.0017,0.0014]} \\
{[-0.0082,0.0073]} \\
0.0000 \\
{[-0.0060,0.0033]} \\
{[-0.0206,0.0131]}\end{array}$ \\
\hline$f_{\Lambda 1}$ & $\begin{array}{l}\text { Approach 1 } \\
f_{a 3}=f_{a 2}=f_{\Lambda 1}^{Z \gamma}=0 \\
\text { Approach 1 } \\
\text { float } f_{a 3}, f_{a 2}, f_{\Lambda 1}^{Z \gamma} \\
\text { Approach 2 } \\
\text { float } f_{a 3}, f_{a 2}\end{array}$ & $\begin{array}{l}\text { best fit } \\
68 \% \mathrm{CL} \\
95 \% \mathrm{CL} \\
\text { best fit } \\
68 \% \mathrm{CL} \\
95 \% \mathrm{CL} \\
\text { best fit } \\
68 \% \mathrm{CL} \\
95 \% \mathrm{CL}\end{array}$ & $\begin{array}{l}0.00004 \\
{[-0.00002,0.00022]} \\
{[-0.00014,0.00060]} \\
0.18629 \\
{[-0.00002,0.00019] \cup[0.07631,0.27515]} \\
{[-0.00523,0.35567]} \\
0.00012 \\
{[-0.00021,0.00141]} \\
{[-0.00184,0.00443]}\end{array}$ & $\begin{array}{l}0.00000 \\
{[-0.00016,0.00026]} \\
{[-0.00069,0.00110]} \\
0.00000 \\
{[-0.00017,0.00036]} \\
{[-0.00076,0.00134]} \\
0.0000 \\
{[-0.0013,0.0030]} \\
{[-0.0056,0.0102]}\end{array}$ \\
\hline$f_{\Lambda 1}^{Z \gamma}$ & $\begin{array}{l}\text { Approach } 1 \\
f_{a 3}=f_{a 2}=f_{\Lambda 1}=0 \\
\text { Approach } 1 \\
\text { float } f_{a 3}, f_{a 2}, f_{\Lambda 1}\end{array}$ & $\begin{array}{l}\text { best fit } \\
68 \% \mathrm{CL} \\
95 \% \mathrm{CL} \\
\text { best fit } \\
68 \% \mathrm{CL} \\
95 \% \mathrm{CL}\end{array}$ & $\begin{array}{l}-0.00001 \\
{[-0.00099,0.00057]} \\
{[-0.00387,0.00301]} \\
-0.02884 \\
{[-0.09000,-0.00534] \cup[-0.00068,0.00078]} \\
{[-0.29091,0.03034]}\end{array}$ & $\begin{array}{l}0.0000 \\
{[-0.0026,0.0020]} \\
{[-0.0096,0.0082]} \\
0.0000 \\
{[-0.0027,0.0026]} \\
{[-0.0099,0.0096]}\end{array}$ \\
\hline
\end{tabular}

assuming top quark dominance in the loop. The results are shown for each coupling separately, with the other three anomalous couplings either set to zero or left unconstrained in the fit. Figure 19 shows the same results presented as two-dimensional contours, where all couplings discussed above are left unconstrained. In all cases, the likelihood scans are limited to the physical range of $\left|f_{a 3}\right|+\left|f_{a 2}\right|+\left|f_{\Lambda 1}\right|+\left|f_{\Lambda 1}^{Z \gamma}\right|<1$.

There are several features visible on these plots. First, the results with all other couplings constrained to zero exhibit narrow minima near $f_{a i}=0$ in both the expected and the observed scans. This effect comes from utilizing production information. The anomalous coupling terms in Eq. (2) are multiplied by a factor of $q_{i}^{2}$, which is larger in VBF and $V H$ production than in $H \rightarrow 4 \ell$ decay. As a result, the cross section in $\mathrm{VBF}$ and $\mathrm{VH}$ production increases quickly with $f_{a i}$. At the same time, the constraints above $f_{a i} \sim 0.02$ are dominated by the decay information from $H \rightarrow 4 \ell$.

However, when all four anomalous couplings are allowed to float independently, the best fit value is $\left(f_{a 3}, f_{a 2}, f_{\Lambda 1}\right.$, $\left.f_{\Lambda 1}^{Z \gamma}\right)=(-0.00805,-0.24679,0.18629,-0.02884)$. This global minimum is driven by the decay information from $H \rightarrow 4 \ell$ and is only slightly preferred to the local minimum at $(0,0,0,0)$, with a difference in $-2 \ln (\mathcal{L})$ of 0.05 between the $\mathrm{SM}$ value and the global minimum. The local minimum at $(0,0,0,0)$ is still evident in the four-dimensional distribution and its projections on each parameter, and is driven by the production information, as discussed above for 

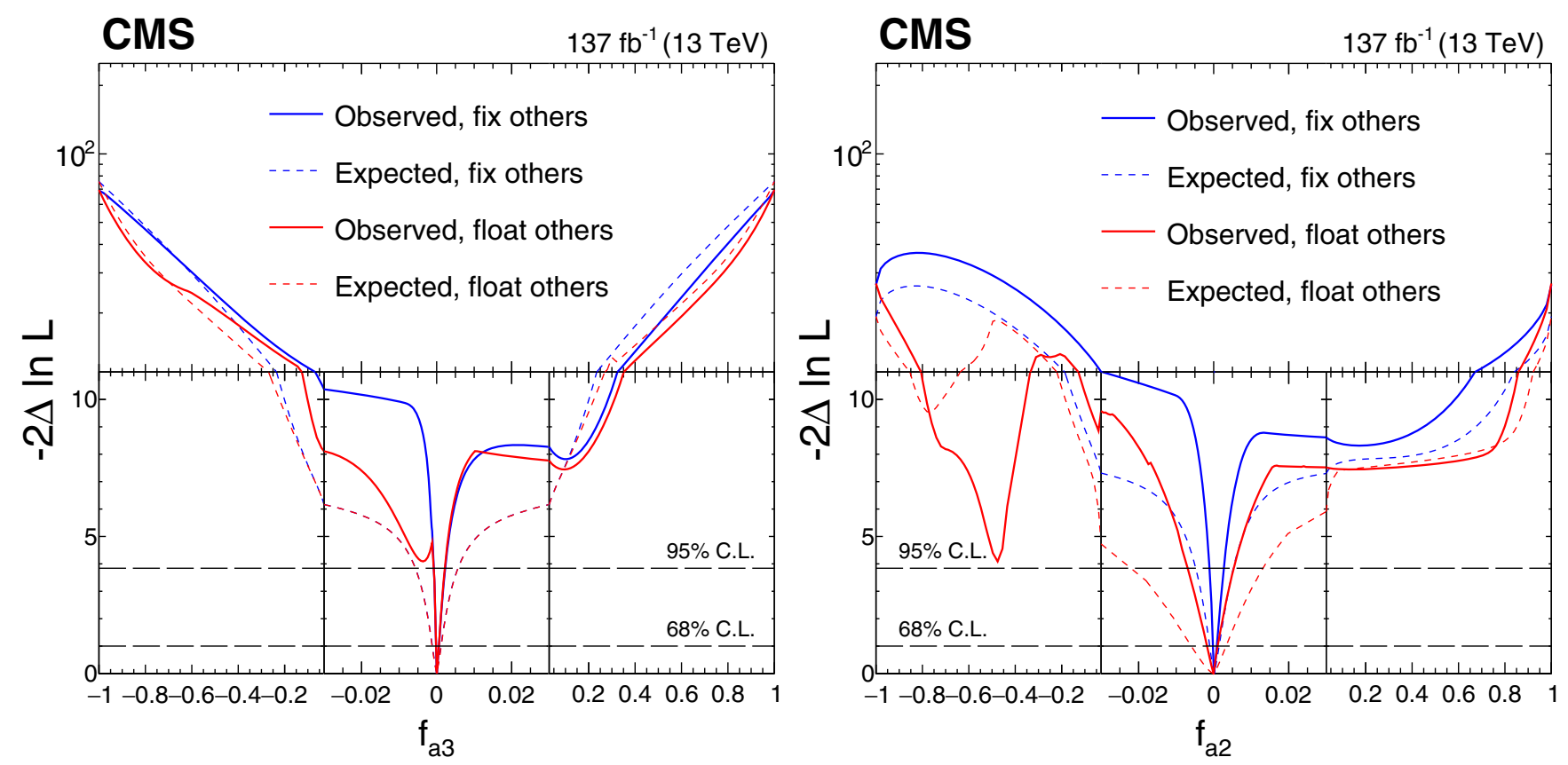

CMS

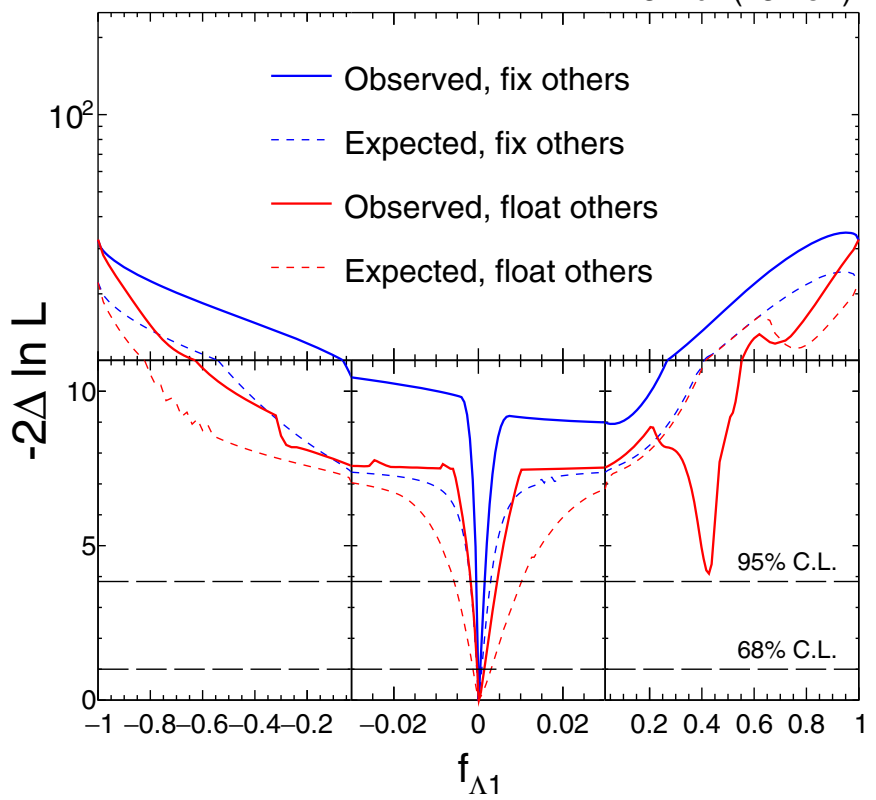

FIG. 20. Observed (solid) and expected (dashed) likelihood scans of $f_{a 3}$ (upper left), $f_{a 2}$ (upper right), and $f_{\Lambda 1}$ (lower) in approach 2 within SMEFT with the symmetry relationship of couplings set in Eqs. (3)-(7). The results are shown for each coupling separately with the other anomalous coupling fractions either set to zero or left unconstrained in the fit. In all cases, the signal strength parameters have been left unconstrained. The dashed horizontal lines show the 68 and 95\% CL regions. For better visibility of all features, the $x$ and $y$ axes are presented with variable scales. On the linear-scale $x$ axis, an enlargement is applied in the range -0.03 to 0.03 . The $y$ axis is shown in linear or logarithmic scale for values of $-2 \ln \mathcal{L}$ below or above 11 , respectively.

the fits with one parameter. Owing to what appears to be a statistical fluctuation in the observed data when the $-2 \ln (\mathcal{L})$ minima obtained from the decay and from the production kinematics differ, the observed constraints appear weaker than expected. However, the results are still statistically consistent with the SM and with the expected constraints in the SM. Should the global minimum nonetheless persist away from $(0,0,0,0)$ with more data, it will be interesting to study consistency of the constraints from the VBF and $V H$ production and from the $H \rightarrow 4 \ell$ decay. The production and decay test different ranges of $q_{i}^{2}$, as discussed above. If the $q_{i}^{2}$ growth is truncated in the VBF and $V H$ production due to lower-energy BSM effects, then the decay information becomes more important. 
TABLE VII. The observed correlation coefficients of the signal strength $\mu_{V}$ and the $f_{a 3}, f_{a 2}, f_{\Lambda 1}$ parameters in approach 2 within SMEFT with the symmetry relationship of couplings set in Eqs. (3)-(7).

\begin{tabular}{lrrrr}
\hline \hline Parameter & & \multicolumn{2}{c}{ Observed correlation } \\
\hline & $\mu_{V}$ & $f_{a 3}$ & $f_{a 2}$ & $f_{\Lambda 1}$ \\
$\mu_{V}$ & 1 & -0.242 & -0.060 & -0.025 \\
$f_{a 3}$ & & 1 & -0.082 & +0.008 \\
$f_{a 2}$ & & 1 & -0.763 \\
$f_{\Lambda 1}$ & & & & 1 \\
\hline \hline
\end{tabular}

\section{E. Constraints on $H V V$ couplings within $S U(2) \times U(1)$ symmetry}

The above studies of the $H$ boson couplings to EW vector bosons are repeated following approach 2 within SMEFT with the symmetries in Eqs. (3)-(7). In this case, the $f_{\Lambda 1}^{Z \gamma}$ parameter is not independent. Therefore, constraints on the three parameters $f_{a 3}, f_{a 2}, f_{\Lambda 1}$, and the signal strength are obtained in this scenario following the same approach as above. These constraints are shown in Fig. 20 and Table VI. The measured signal strength is
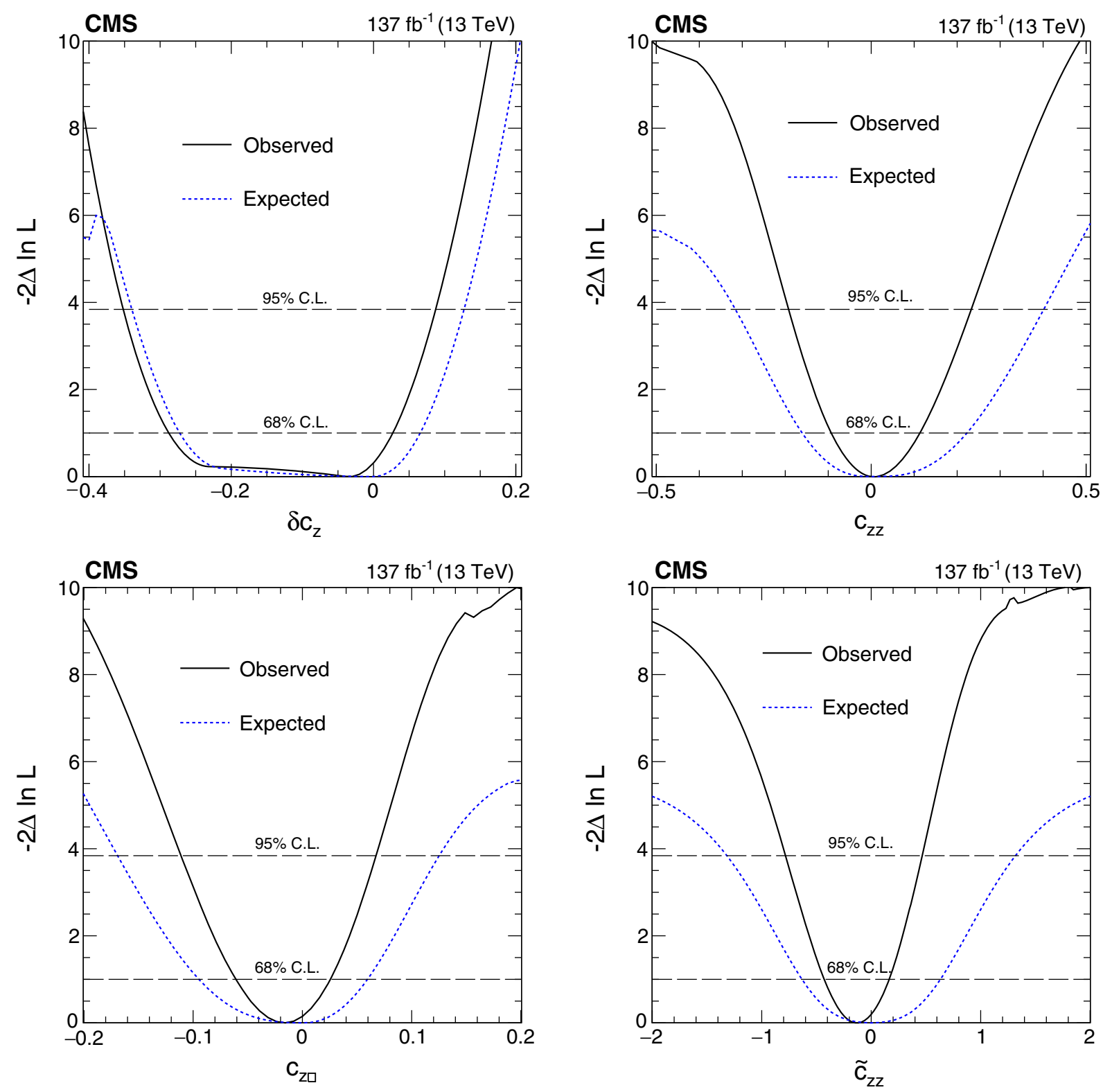

FIG. 21. Observed (solid) and expected (dashed) constraints from a simultaneous fit of the SMEFT parameters $\delta c_{\mathrm{z}}$ (upper left), $c_{\mathrm{zZ}}$ (upper right), $c_{\mathrm{z} \square}$ (lower left), and $\tilde{c}_{\mathrm{zz}}$ (lower right) with the $c_{g g}$ and $\tilde{c}_{g g}$ couplings left unconstrained. 

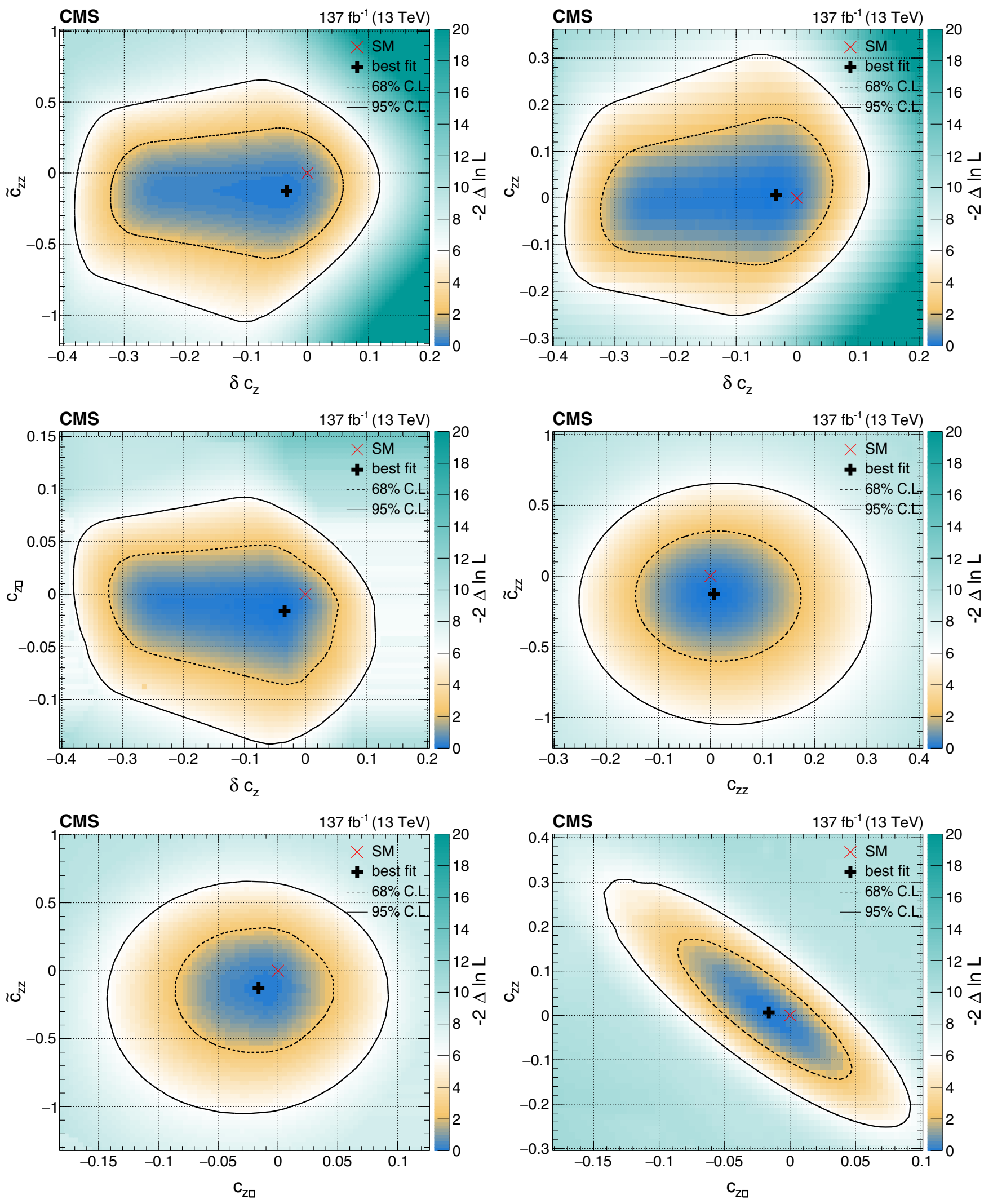

FIG. 22. Observed two-dimensional constraints from a simultaneous fit of the SMEFT parameters $\delta c_{\mathrm{z}}, c_{\mathrm{zz}}, c_{\mathrm{z} \square}$, and $\tilde{c}_{\mathrm{zz}}$ with the $c_{g g}$ and $\tilde{c}_{g g}$ couplings left unconstrained. 
TABLE VIII. Summary of constraints on the $H t t, H g g$, and $H V V$ coupling parameters in the Higgs basis of SMEFT. The observed correlation coefficients are presented for the $H t t$ and $H g g$ and $H V V$ couplings in the fit configurations discussed in text and shown in Figs. 17 and 22, respectively.

\begin{tabular}{|c|c|c|c|c|c|c|c|}
\hline \multirow[t]{2}{*}{ Channels } & \multirow[t]{2}{*}{ Coupling } & \multirow[t]{2}{*}{ Observed } & \multirow[t]{2}{*}{ Expected } & \multicolumn{4}{|c|}{ Observed correlation } \\
\hline & & & & $c_{g g}$ & $\tilde{c}_{g g}$ & $\kappa_{t}$ & $\tilde{\kappa}_{t}$ \\
\hline$t H \& t \bar{t} H \& g g H$ & $c_{g g}$ & $-0.0012_{-0.0174}^{+0.0022}$ & $0.0000_{-0.0196}^{+0.0019}$ & 1 & -0.050 & -0.941 & +0.029 \\
\hline & $\tilde{c}_{g g}$ & $-0.0017_{-0.0130}^{+0.0160}$ & $0.0000_{-0.0138}^{+0.0138}$ & & 1 & +0.046 & -0.568 \\
\hline & $\kappa_{t}$ & $1.05_{-0.20}^{+0.25}$ & $1.00_{-0.26}^{+0.34}$ & & & 1 & +0.168 \\
\hline & $\tilde{\kappa}_{t}$ & $-0.01_{-0.67}^{+0.69}$ & $0.00_{-0.71}^{+0.71}$ & & & & 1 \\
\hline \multirow{5}{*}{$\mathrm{VBF} \& V H \& H \rightarrow 4 \ell$} & & & & $\delta c_{\mathrm{z}}$ & $c_{\mathrm{zZ}}$ & $c_{\mathrm{z} \square}$ & $\tilde{c}_{\mathrm{ZZ}}$ \\
\hline & $\delta c_{\mathrm{z}}$ & $-0.03_{-0.25}^{+0.06}$ & $0.00_{-0.27}^{+0.07}$ & 1 & +0.241 & -0.060 & -0.009 \\
\hline & $c_{\mathrm{zZ}}$ & $0.01_{-0.10}^{+0.11}$ & $0.00_{-0.16}^{+0.22}$ & & 1 & -0.884 & +0.058 \\
\hline & $c_{\mathrm{z} \square}$ & $-0.02_{-0.04}^{+0.04}$ & $0.00_{-0.09}^{+0.06}$ & & & 1 & +0.020 \\
\hline & $\tilde{c}_{\mathrm{zz}}$ & $-0.11_{-0.31}^{+0.04}$ & $0.00_{-0.63}^{+0.63}$ & & & & 1 \\
\hline
\end{tabular}

$\mu_{V}=1.10_{-0.42}^{+0.50}$. The observed correlation coefficients are shown in Table VII. Keeping only linear terms and dropping terms with order greater than one for anomalous couplings does not allow us to make a reasonable likelihood scan, since the probability density goes negative, as discussed in Sec. II C.

Since the relationship of the $H W W$ and $H Z Z$ couplings does not affect the measurement of the $f_{a 3}$ parameter in the $H \rightarrow 4 \ell$ decay, the constraints from the decay information in the wider range of $f_{a 3}$ in approach 2 are unaffected compared to approach 1, when other couplings are fixed to zero. However, with one less parameter to float, the constraints are modified somewhat when all other couplings are left unconstrained. The modified relationship between the $H W W$ and $H Z Z$ couplings also leads to some modification of constraints using production information in the narrow range of $f_{a 3}$. On the other hand, the $f_{a 2}$ and $f_{\Lambda 1}$ parameters are modified substantially because the $f_{\Lambda 1}^{Z \gamma}$

TABLE IX. Summary of constraints on the $H V V$ coupling parameters in the Warsaw basis of SMEFT. For each coupling constraint reported, three other independent operators are left unconstrained, where only one of the three operators $c_{H W}, c_{H W \mathrm{~B}}$, and $c_{H \mathrm{~B}}$ is independent, and only one of $c_{H \tilde{W}}, c_{H \tilde{W} \mathrm{~B}}$, and $c_{H \tilde{\mathrm{B}}}$ is independent.

\begin{tabular}{lcrr}
\hline \hline Channels & Coupling & Observed & Expected \\
\hline VBF \& VH \& $H \rightarrow 4 \ell$ & $c_{H \square}$ & $0.04_{-0.45}^{+0.43}$ & $0.00_{-0.93}^{+0.75}$ \\
& $c_{H \mathrm{D}}$ & $-0.73_{-4.21}^{+0.97}$ & $0.00_{-4.60}^{+1.06}$ \\
& $c_{H W}$ & $0.01_{-0.17}^{+0.18}$ & $0.00_{-0.28}^{+0.39}$ \\
& $c_{H W \mathrm{~B}}$ & $0.01_{-0.18}^{+0.20}$ & $0.00_{-0.31}^{+0.42}$ \\
& $c_{H \mathrm{~B}}$ & $0.00_{-0.05}^{+0.05}$ & $0.00_{-0.08}^{+0.03}$ \\
& $c_{H \tilde{W}}$ & $-0.23_{-0.52}^{+0.51}$ & $0.00_{-1.11}^{+1.11}$ \\
& $c_{H \tilde{\mathrm{B}}}$ & $-0.25_{-0.57}^{+0.56}$ & $0.00_{-1.21}^{+1.21}$ \\
& $c_{H \tilde{\mathrm{B}}}$ & $-0.06_{-0.16}^{+0.15}$ & $0.00_{-0.33}^{+0.33}$ \\
\hline \hline
\end{tabular}

information gets absorbed into these measurements through symmetry relationships.

The measurement of the signal strength $\mu_{V}$ and the $f_{a 3}$, $f_{a 2}, f_{\Lambda 1}$ parameters can be reinterpreted in terms of the $\delta c_{\mathrm{z}}, \quad c_{\mathrm{zZ}}, \quad c_{\mathrm{z} \square}$, and $\tilde{c}_{\mathrm{zz}}$ coupling strength parameters. Observed one- and two-dimensional constraints from a simultaneous fit of SMEFT parameters are shown in Figs. 21 and 22. The $c_{g g}$ and $\tilde{c}_{g g}$ couplings are left unconstrained. A summary of all constraints on the Htt, $H g g$, and $H V V$ coupling parameters in the Higgs basis of SMEFT, including the correlation coefficients, is shown in Table VIII. The results in this table are taken from Secs. VI C and VIE, as measured in the $t H, t \bar{t} H, g g H$, and EW processes.

The above interpretation of $H V V$ results in terms of the $\delta c_{\mathrm{z}}, c_{\mathrm{zz}}, c_{\mathrm{z} \square}$, and $\tilde{c}_{\mathrm{zz}}$ couplings can be extended into an interpretation in terms of the couplings in the Warsaw SMEFT basis [28]. In this basis, nine operators are considered: $c_{H \square}, c_{H \mathrm{D}}, c_{H W}, c_{H W \mathrm{~B}}, c_{H \mathrm{~B}}, c_{H \tilde{W}}, c_{H \tilde{W} \mathrm{~B}}$, $c_{H \tilde{\mathrm{B}}}$, and $\delta_{v}$, where the latter is a linear combination of additional Warsaw basis operators [71]. However, not all nine of these operators are independent. First of all, consideration of Eq. (3) leads to $\delta_{v}$ expression as a linear combination of $c_{H \mathrm{D}}$ and $c_{H W \mathrm{~B}}$. Four constraints on the couplings $a_{2}^{\gamma \gamma, Z \gamma}$ and $a_{3}^{\gamma \gamma, Z \gamma}$ lead to only one of the three operators $c_{H W}, c_{H W \mathrm{~B}}$, and $c_{H \mathrm{~B}}$ being independent, and only one of $c_{H \tilde{W}}, c_{H \tilde{W} \mathrm{~B}}$, and $c_{H \tilde{\mathrm{B}}}$ being independent. Therefore, we obtain only four independent constraints, the same number as in the Higgs basis. We note that the couplings of the $Z$ boson to fermions are fixed to those expected in the SM because those are well constrained from prior measurements and this constraint is already included in our primary measurements. Even though some of the above EFT operators may affect couplings of the $Z$ boson, their effect must be compensated by the other EFT operators not affecting the $H$ boson couplings directly. With the above constraints, we use the tools in Refs. [33,71] to relate 
operators in the Higgs and Warsaw bases. Since it is arbitrary which one of the three operators is considered to be independent, we present results with all three choices in each case. The results can be found in Table IX, where three other independent couplings are left unconstrained for each measurement.

\section{SUMMARY}

In this paper, a comprehensive study of $C P$ violation, anomalous couplings, and the tensor structure of $H$ boson interactions with electroweak gauge bosons, gluons, and fermions, using all accessible production mechanisms and the $H \rightarrow 4 \ell$ decay mode, is presented. The results are based on the 2016-2018 data from $p p$ collisions recorded with the CMS detector during run 2 of the LHC, corresponding to an integrated luminosity of $137 \mathrm{fb}^{-1}$ at a center-of-mass energy of $13 \mathrm{TeV}$. These results significantly surpass our results from run 1 [13] in both precision and coverage. The improvements result not only from a significantly increased sample of $H$ bosons but also from a detailed analysis of kinematic distributions of the particles associated with the $H$ boson production in addition to kinematic distributions in its decay. These results also surpass our earlier studies of on shell production of the $H$ boson in this decay channel with a partial run 2 dataset $[16,17]$.

The parametrization of the $H$ boson production and decay processes is based on a scattering amplitude or, equivalently, an effective field theory Lagrangian, with operators up to dimension six. Additional symmetries and prior measurements allow us to reduce the number of independent parameters and make a connection to the standard model effective field theory (SMEFT) formulation. Dedicated Monte Carlo programs and matrixelement reweighting techniques provide modeling of all kinematic effects in the production and decay of the $H$ boson, with any variation of parameters of the scattering amplitude and with full simulation of detector effects. Each production process of the $H$ boson is identified using the kinematic features of its associated particles. The MELA is employed to construct observables that are optimal for the measurement of the targeted anomalous couplings in each process, including $C P$-sensitive observables. A maximum likelihood fit allows a simultaneous measurement of up to five $H V V$, two $H g g$, and two $H t t$ couplings.

For the first time, we present a complete and dedicated study of $C P$ properties in the $H$ boson coupling to gluons through a loop of heavy particles using the $C P$-sensitive observables, while separating the electroweak and strong boson fusion processes. An interpretation of the loop contribution is made both with and without an assumption of top quark dominance, which allows for a new heavy particle to contribute. In both cases, combination with the $C P$-sensitive measurement of the $H t t$ coupling in the $t \bar{t} H$ and $t H$ processes allows either simultaneous or separate measurements of the two effective pointlike $H g g$ couplings and the two $\mathrm{Htt}$ couplings, both $\mathrm{CP}$-odd and $\mathrm{CP}$-even. For the $t \bar{t} H$ and $t H$ processes, results in the $H \rightarrow 4 \ell$ channel are combined with those in the $H \rightarrow \gamma \gamma$ channel [26]. This is the first comprehensive study of $C P$ properties in the $\mathrm{Hgg}$ and $\mathrm{Htt}$ couplings from a simultaneous measurement of the $g g H, t \bar{t} H$, and $t H$ processes.

Also for the first time, we present the measurement of $C P$ properties and the tensor structure of the $H$ boson's interactions with two electroweak bosons with up to five parameters measured simultaneously. The $H V V$ coupling is analyzed in VBF and $V H$ production and in $H \rightarrow V V \rightarrow 4 \ell$ decay. The measurements are performed with two approaches. In the first approach, more lenient symmetry considerations are applied, which allow a less restrictive interpretation of results. In the second approach, $\mathrm{SU}(2) \times \mathrm{U}(1)$ symmetry is invoked and the formulation becomes equivalent to SMEFT. The operator basis is chosen to coincide with the couplings of the mass eigenstates, which allows us to minimize the number of independent parameters. A translation of the SMEFT results to the Warsaw basis is also presented for easier comparison with other results.

In all cases, we first present results in terms of the total cross section of a process and the fractional contribution of each anomalous coupling. These results are further reinterpreted in terms of direct constraints on the couplings by applying certain assumptions about the $H$ boson total width. Each of the measurements presented here is limited by statistical precision and is consistent with the expectations for the standard model Higgs boson.

\section{ACKNOWLEDGMENTS}

We thank Markus Schulze for optimizing the JHUGen Monte Carlo simulation program and matrix element library for this analysis. We thank Amitabh Basu for guidance on implementing the cutting planes algorithm and Tianran Chen for updating the HOM4PS program for this analysis and providing support. We are grateful to the members of the LHC Higgs and EFT Working Groups for stimulating the development of several phenomenological aspects of this work, among which is relating the EFT operator bases for the Higgs boson couplings. We congratulate our colleagues in the CERN accelerator departments for the excellent performance of the LHC and thank the technical and administrative staffs at CERN and at other CMS institutes for their contributions to the success of the CMS effort. In addition, we gratefully acknowledge the computing centers and personnel of the Worldwide LHC Computing Grid and other centers for delivering so effectively the computing infrastructure essential to our analyses. We also acknowledge the Maryland Advanced Research Computing Center (MARCC) for providing computing resources essential for this analysis. Finally, we acknowledge the enduring support for the construction and operation of the LHC, the CMS detector, and the supporting computing infrastructure provided by the following funding agencies: the Austrian Federal 
Ministry of Education, Science and Research and the Austrian Science Fund; the Belgian Fonds de la Recherche Scientifique, and Fonds voor Wetenschappelijk Onderzoek; the Brazilian Funding Agencies (CNPq, CAPES, FAPERJ, FAPERGS, and FAPESP); the Bulgarian Ministry of Education and Science; CERN; the Chinese Academy of Sciences, Ministry of Science and Technology, and National Natural Science Foundation of China; the Ministerio de Ciencia Tecnología e Innovación (MINCIENCIAS), Colombia; the Croatian Ministry of Science, Education and Sport, and the Croatian Science Foundation; the Research and Innovation Foundation, Cyprus; the Secretariat for Higher Education, Science, Technology and Innovation, Ecuador; the Ministry of Education and Research, Estonian Research Council via Grants No. PRG780, No. PRG803, and No. PRG445 and European Regional Development Fund, Estonia; the Academy of Finland, Finnish Ministry of Education and Culture, and Helsinki Institute of Physics; the Institut National de Physique Nucléaire et de Physique des Particules / CNRS, and Commissariat à l'Énergie Atomique et aux Énergies Alternatives / CEA, France; the Bundesministerium für Bildung und Forschung, the Deutsche Forschungsgemeinschaft (DFG), under Germany's Excellence Strategy-EXC 2121 "Quantum Universe"-Grant No. 390833306, and under Project No. 400140256-GRK2497, and HelmholtzGemeinschaft Deutscher Forschungszentren, Germany; the General Secretariat for Research and Technology, Greece; the National Research, Development and Innovation Fund, Hungary; the Department of Atomic Energy and the Department of Science and Technology, India; the Institute for Studies in Theoretical Physics and Mathematics, Iran; the Science Foundation, Ireland; the Istituto Nazionale di Fisica Nucleare, Italy; the Ministry of Science, ICT and Future Planning, and National Research Foundation (NRF), Republic of Korea; the Ministry of Education and Science of the Republic of Latvia; the Lithuanian Academy of Sciences; the Ministry of Education, and University of Malaya (Malaysia); the Ministry of Science of Montenegro; the Mexican Funding Agencies (BUAP, CINVESTAV, CONACYT, LNS, SEP, and UASLP-FAI); the Ministry of Business, Innovation and Employment, New Zealand; the Pakistan Atomic Energy Commission; the Ministry of Science and HigherEducation and the National Science Center, Poland; the Fundação para a Ciência e a Tecnologia, Portugal; JINR, Dubna; the Ministry of Education and Science of the Russian Federation, the Federal Agency of Atomic Energy of the Russian Federation, Russian Academy of Sciences, the Russian Foundation for Basic Research, and the National Research Center "Kurchatov Institute"; the Ministry of Education, Science and Technological Development of Serbia; the Secretaría de Estado de Investigación, Desarrollo e Innovación, Programa Consolider-Ingenio 2010, Plan Estatal de Investigación Científica y Técnica y de Innovación 20172020, research Project No. IDI-2018-000174 del Principado de Asturias, and Fondo Europeo de Desarrollo Regional, Spain; the Ministry of Science, Technology and Research, Sri Lanka; the Swiss Funding Agencies (ETH Board, ETH Zurich, PSI, SNF, UniZH, Canton Zurich, and SER); the Ministry of Science and Technology, Taipei; the Thailand Center of Excellence in Physics, the Institute for the Promotion of Teaching Science and Technology of Thailand, Special Task Force for Activating Research and the National Science and Technology Development Agency of Thailand; the Scientific and Technical Research Council of Turkey, and Turkish Atomic Energy Authority; the National Academy of Sciences of Ukraine; the Science and Technology Facilities Council, UK; the US Department of Energy, and the US National Science Foundation. Individuals have received support from the Marie-Curie program and the European Research Council and Horizon 2020 Grant, Contract No. 675440, No. 724704, No. 752730, No. 765710, and No. 824093 (European Union); the Leventis Foundation; the Alfred P. Sloan Foundation; the Alexander von Humboldt Foundation; the Belgian Federal Science Policy Office; the Fonds pour la Formation à la Recherche dans l'Industrie et dans l'Agriculture (FRIABelgium); the Agentschap voor Innovatie door Wetenschap en Technologie (IWT-Belgium); the F. R. S.-FNRS and FWO (Belgium) under the "Excellence of Science-EOS"-be.h Project No. 30820817; the Beijing Municipal Science \& Technology Commission, No. Z191100007219010; the Ministry of Education, Youth and Sports (MEYS) of the Czech Republic; the Lendület ("Momentum") Program and the János Bolyai Research Scholarship of the Hungarian Academy of Sciences, the New National Excellence Program ÚNKP, the NKFIA research Grants No. 123842, No. 123959 , No. 124845 , No. 124850 , No. 125105 , No. 128713, No. 128786, and No. 129058 (Hungary); the Council of Scientific and Industrial Research, India; the National Science Center (Poland), Contracts Opus No. 2014/15/B/ST2/03998 and No. 2015/19/B/ST2/02861; the National Priorities Research Program by Qatar National Research Fund; the Ministry of Science and Higher Education, Project No. 0723-2020-0041 (Russia); the Programa de Excelencia María de Maeztu, and the Programa Severo Ochoa del Principado de Asturias; the Thalis and Aristeia programs cofinanced by EU-ESF, and the Greek NSRF; the Rachadapisek Sompot Fund for Postdoctoral Fellowship, Chulalongkorn University, and the Chulalongkorn Academic into Its 2nd Century Project Advancement Project (Thailand); the Kavli Foundation; the Nvidia Corporation; the SuperMicro Corporation; the Welch Foundation, Contract No. C-1845; and the Weston Havens Foundation (USA). 
[1] ATLAS Collaboration, Observation of a new particle in the search for the Standard Model Higgs boson with the ATLAS detector at the LHC, Phys. Lett. B 716, 1 (2012).

[2] CMS Collaboration, Observation of a new boson at a mass of $125 \mathrm{GeV}$ with the CMS experiment at the LHC, Phys. Lett. B 716, 30 (2012).

[3] CMS Collaboration, Observation of a new boson with mass near $125 \mathrm{GeV}$ in $p p$ collisions at $\sqrt{s}=7$ and $8 \mathrm{TeV}$, J. High Energy Phys. 06 (2013) 081.

[4] S. L. Glashow, Partial-symmetries of weak interactions, Nucl. Phys. 22, 579 (1961).

[5] F. Englert and R. Brout, Broken Symmetry and the Mass of Gauge Vector Mesons, Phys. Rev. Lett. 13, 321 (1964).

[6] P. W. Higgs, Broken Symmetries, Massless Particles and Gauge Fields, Phys. Lett. 12, 132 (1964).

[7] P. W. Higgs, Broken Symmetries and the Masses of Gauge Bosons, Phys. Rev. Lett. 13, 508 (1964).

[8] G. S. Guralnik, C. R. Hagen, and T. W. B. Kibble, Global Conservation Laws and Massless Particles, Phys. Rev. Lett. 13, 585 (1964).

[9] S. Weinberg, A Model of Leptons, Phys. Rev. Lett. 19, 1264 (1967).

[10] A. Salam, Weak and electromagnetic interactions, in Elementary Particle Physics: Relativistic Groups and Analyticity, edited by N. Svartholm (Almqvist \& Wiksell, Stockholm, 1968), p. 367, Proceedings of the Eighth Nobel Symposium.

[11] CMS Collaboration, On the Mass and Spin-Parity of the Higgs Boson Candidate Via Its Decays to $Z$ Boson Pairs, Phys. Rev. Lett. 110, 081803 (2013).

[12] CMS Collaboration, Measurement of the properties of a Higgs boson in the four-lepton final state, Phys. Rev. D 89, 092007 (2014).

[13] CMS Collaboration, Constraints on the spin-parity and anomalous $H V V$ couplings of the Higgs boson in proton collisions at 7 and 8 TeV, Phys. Rev. D 92, 012004 (2015).

[14] CMS Collaboration, Limits on the Higgs boson lifetime and width from its decay to four charged leptons, Phys. Rev. D 92, 072010 (2015).

[15] CMS Collaboration, Combined search for anomalous pseudoscalar $H V V$ couplings in $V H(H \rightarrow b \bar{b})$ production and $H \rightarrow V V$ decay, Phys. Lett. B 759, 672 (2016).

[16] CMS Collaboration, Constraints on anomalous Higgs boson couplings using production and decay information in the four-lepton final state, Phys. Lett. B 775, 1 (2017).

[17] CMS Collaboration, Measurements of the Higgs boson width and anomalous HVV couplings from on-shell and off-shell production in the four-lepton final state, Phys. Rev. D 99, 112003 (2019).

[18] CMS Collaboration, Constraints on anomalous $H V V$ couplings from the production of Higgs bosons decaying to $\tau$ lepton pairs, Phys. Rev. D 100, 112002 (2019).

[19] ATLAS Collaboration, Evidence for the spin-0 nature of the Higgs boson using ATLAS data, Phys. Lett. B 726, 120 (2013).

[20] ATLAS Collaboration, Study of the spin and parity of the Higgs boson in diboson decays with the ATLAS detector, Eur. Phys. J. C 75, 476 (2015).

[21] ATLAS Collaboration, Test of $C P$ invariance in vectorboson fusion production of the Higgs boson using the optimal observable method in the ditau decay channel with the ATLAS detector, Eur. Phys. J. C 76, 658 (2016).

[22] ATLAS Collaboration, Measurement of inclusive and differential cross sections in the $H \rightarrow Z Z^{*} \rightarrow 4 \ell$ decay channel in $p p$ collisions at $\sqrt{s}=13 i \mathrm{TeV}$ with the ATLAS detector, J. High Energy Phys. 10 (2017) 132.

[23] ATLAS Collaboration, Measurement of the Higgs boson coupling properties in the $H \rightarrow Z Z^{*} \rightarrow 4 \ell$ decay channel at $\sqrt{s}=13 \mathrm{TeV}$ with the ATLAS detector, J. High Energy Phys. 03 (2018) 095.

[24] ATLAS Collaboration, Measurements of Higgs boson properties in the diphoton decay channel with $36 \mathrm{fb}^{-1}$ of $p p$ collision data at $\sqrt{s}=13 \mathrm{TeV}$ with the ATLAS detector, Phys. Rev. D 98, 052005 (2018).

[25] ATLAS Collaboration, Test of $C P$ invariance in vectorboson fusion production of the Higgs boson in the $\mathrm{H} \rightarrow \tau \tau$ channel in proton-proton collisions at $\sqrt{s}=13 \mathrm{TeV}$ with the ATLAS detector, Phys. Lett. B 805, 135426 (2020).

[26] CMS Collaboration, Measurements of $t \bar{t} H$ Production and the $C P$ Structure of the Yukawa Interaction between the Higgs Boson and Top Quark in the Diphoton Decay Channel, Phys. Rev. Lett. 125, 061801 (2020).

[27] ATLAS Collaboration, CP Properties of Higgs Boson Interactions with Top Quarks in the $t \bar{t} H$ and $t H$ Processes Using $H \rightarrow \gamma \gamma$ with the ATLAS Detector, Phys. Rev. Lett. 125, 061802 (2020).

[28] LHC Higgs Cross Section Working Group, Handbook of LHC Higgs cross sections: 4. Deciphering the nature of the Higgs sector, CERN, 2016, https://doi.org/10.23731/ CYRM-2017-002.

[29] Y. Gao, A. V. Gritsan, Z. Guo, K. Melnikov, M. Schulze, and N. V. Tran, Spin determination of single-produced resonances at hadron colliders, Phys. Rev. D 81, 075022 (2010).

[30] S. Bolognesi, Y. Gao, A. V. Gritsan, K. Melnikov, M. Schulze, N. V. Tran, and A. Whitbeck, Spin and parity of a single-produced resonance at the LHC, Phys. Rev. D 86, 095031 (2012).

[31] I. Anderson, S. Bolognesi, F. Caola, Y. Gao, A. V. Gritsan, C. B. Martin, K. Melnikov, M. Schulze, N. V. Tran, A. Whitbeck, and Y. Zhou, Constraining anomalous $H V V$ interactions at proton and lepton colliders, Phys. Rev. D 89, 035007 (2014).

[32] A. V. Gritsan, R. Röntsch, M. Schulze, and M. Xiao, Constraining anomalous Higgs boson couplings to the heavy flavor fermions using matrix element techniques, Phys. Rev. D 94, 055023 (2016).

[33] A. V. Gritsan, J. Roskes, U. Sarica, M. Schulze, M. Xiao, and Y. Zhou, New features in the JHU generator framework: Constraining Higgs boson properties from on-shell and offshell production, Phys. Rev. D 102, 056022 (2020).

[34] ATLAS Collaboration, Higgs boson production crosssection measurements and their EFT interpretation in the $4 \ell$ decay channel at $\sqrt{s}=13 \mathrm{TeV}$ with the ATLAS detector, Eur. Phys. J. C 80, 957 (2020); Erratum, Eur. Phys. J. C 81, 29 (2021).

[35] ATLAS Collaboration, Measurements of the Higgs boson inclusive and differential fiducial cross sections in the $4 \ell$ decay channel at $\sqrt{s}=13 \mathrm{TeV}$, Eur. Phys. J. C 80, 942 (2020). 
[36] C. A. Nelson, Correlation between decay planes in Higgs boson decays into a $W$ Pair (into a $Z$ pair), Phys. Rev. D 37, 1220 (1988).

[37] A. Soni and R. M. Xu, Probing $C P$ violation via Higgs decays to four leptons, Phys. Rev. D 48, 5259 (1993).

[38] D. Chang, W.-Y. Keung, and I. Phillips, $C P$ odd correlation in the decay of neutral Higgs boson into $\mathrm{ZZ}, W^{+} W^{-}$, or $t \bar{t}$, Phys. Rev. D 48, 3225 (1993).

[39] V. D. Barger, K.-m. Cheung, A. Djouadi, B. A. Kniehl, and P. M. Zerwas, Higgs bosons: Intermediate mass range at e+ e- colliders, Phys. Rev. D 49, 79 (1994).

[40] T. Arens and L. M. Sehgal, Energy spectra and energy correlations in the decay $H \rightarrow Z Z \rightarrow \mu+\mu-\mu+\mu-$, Z. Phys. C 66, 89 (1995).

[41] S. Bar-Shalom, D. Atwood, G. Eilam, R. R. Mendel, and A. Soni, Large tree level $C P$ violation in $e^{+} e^{-} \rightarrow t \bar{t} H^{0}$ in the two Higgs doublet model, Phys. Rev. D 53, 1162 (1996).

[42] J. F. Gunion and X.-G. He, Determining the $C P$ Nature of a Neutral Higgs Boson at the LHC, Phys. Rev. Lett. 76, 4468 (1996).

[43] T. Han and J. Jiang, $C P$ violating $Z Z H$ coupling at $e^{+} e^{-}$ linear colliders, Phys. Rev. D 63, 096007 (2001).

[44] T. Plehn, D. L. Rainwater, and D. Zeppenfeld, Determining the Structure of Higgs Couplings at the LHC, Phys. Rev. Lett. 88, 051801 (2002).

[45] S. Y. Choi, D. J. Miller, M. M. Mühlleitner, and P. M. Zerwas, Identifying the Higgs spin and parity in decays to Z pairs, Phys. Lett. B 553, 61 (2003).

[46] C. P. Buszello, I. Fleck, P. Marquard, and J. J. van der Bij, Prospective analysis of spin- and $C P$-sensitive variables in $H \rightarrow Z Z \rightarrow \ell_{1}^{+} \ell_{1}^{-} \ell_{2}^{+} \ell_{2}^{-}$at the LHC, Eur. Phys. J. C 32, 209 (2004).

[47] V. Hankele, G. Klamke, D. Zeppenfeld, and T. Figy, Anomalous Higgs boson couplings in vector boson fusion at the CERN LHC, Phys. Rev. D 74, 095001 (2006).

[48] E. Accomando et al., Workshop on $C P$ studies and nonstandard Higgs physics (2006), https://doi.org/10.5170/ CERN-2006-009.

[49] R. M. Godbole, D. J. Miller, and M. M. Mühlleitner, Aspects of $C P$ violation in the $H Z Z$ coupling at the LHC, J. High Energy Phys. 12 (2007) 031.

[50] K. Hagiwara, Q. Li, and K. Mawatari, Jet angular correlation in vector-boson fusion processes at hadron colliders, J. High Energy Phys. 07, 101 (2009).

[51] A. De Rújula, J. Lykken, M. Pierini, C. Rogan, and M. Spiropulu, Higgs look-alikes at the LHC, Phys. Rev. D 82, 013003 (2010).

[52] N. D. Christensen, T. Han, and Y. Li, Testing $C P$ violation in $\mathrm{ZZH}$ interactions at the LHC, Phys. Lett. B 693, 28 (2010).

[53] J. S. Gainer, K. Kumar, I. Low, and R. Vega-Morales, Improving the sensitivity of Higgs boson searches in the golden channel, J. High Energy Phys. 11 (2011) 027.

[54] J. Ellis, D. S. Hwang, V. Sanz, and T. You, A fast track towards the 'Higgs' spin and parity, J. High Energy Phys. 11 (2012) 134.

[55] Y. Chen, N. Tran, and R. Vega-Morales, Scrutinizing the Higgs signal and background in the $2 e 2 \mu$ golden channel, J. High Energy Phys. 01 (2013) 182.
[56] J. S. Gainer, J. Lykken, K. T. Matchev, S. Mrenna, and M. Park, Geolocating the Higgs Boson Candidate at the LHC, Phys. Rev. Lett. 111, 041801 (2013).

[57] P. Artoisenet, P. de Aquino, F. Demartin, R. Frederix, S. Frixione, F. Maltoni, M. K. Mandal, P. Mathews, K. Mawatari, V. Ravindran, S. Seth, P. Torrielli, and M. Zaro, A framework for Higgs characterisation, J. High Energy Phys. 11 (2013) 043.

[58] M. Chen, T. Cheng, J. S. Gainer, A. Korytov, K. T. Matchev, P. Milenovic, G. Mitselmakher, M. Park, A. Rinkevicius, and M. Snowball, Role of interference in unraveling the $Z Z$ couplings of the newly discovered boson at the LHC, Phys. Rev. D 89, 034002 (2014).

[59] Y. Chen and R. Vega-Morales, Extracting effective Higgs couplings in the golden channel, J. High Energy Phys. 04 (2014) 057.

[60] J. S. Gainer, J. Lykken, K. T. Matchev, S. Mrenna, and M. Park, Beyond geolocating: Constraining higher dimensional operators in $H \rightarrow 4 \ell$ with off-shell production and more, Phys. Rev. D 91, 035011 (2015).

[61] M. Gonzalez-Alonso, A. Greljo, G. Isidori, and D. Marzocca, Pseudo-observables in Higgs decays, Eur. Phys. J. C 75, 128 (2015).

[62] M. J. Dolan, P. Harris, M. Jankowiak, and M. Spannowsky, Constraining $C P$-violating Higgs sectors at the LHC using gluon fusion, Phys. Rev. D 90, 073008 (2014).

[63] F. Demartin, F. Maltoni, K. Mawatari, B. Page, and M. Zaro, Higgs characterisation at NLO in QCD: $C P$ properties of the top-quark Yukawa interaction, Eur. Phys. J. C 74, 3065 (2014).

[64] M. R. Buckley and D. Goncalves, Boosting the Direct $C P$ Measurement of the Higgs-Top Coupling, Phys. Rev. Lett. 116, 091801 (2016).

[65] A. Greljo, G. Isidori, J. M. Lindert, and D. Marzocca, Pseudo-observables in electroweak Higgs production, Eur. Phys. J. C 76, 158 (2016).

[66] I. Brivio, T. Corbett, and M. Trott, The Higgs width in the SMEFT, J. High Energy Phys. 10 (2019) 056.

[67] S. Weinberg, Baryon and Lepton Nonconserving Processes, Phys. Rev. Lett. 43, 1566 (1979).

[68] W. Buchmuller and D. Wyler, Effective Lagrangian analysis of new interactions and flavor conservation, Nucl. Phys. B268, 621 (1986).

[69] C. N. Leung, S. T. Love, and S. Rao, Low-energy manifestations of a new interaction scale: Operator analysis, Z. Phys. C 31, 433 (1986).

[70] A. Dedes, W. Materkowska, M. Paraskevas, J. Rosiek, and K. Suxho, Feynman rules for the Standard Model effective field theory in $\mathrm{R}_{\xi}$-gauges, J. High Energy Phys. 06 (2017) 143.

[71] A. Falkowski, B. Fuks, K. Mawatari, K. Mimasu, F. Riva, and V. Sanz, Rosetta: An operator basis translator for Standard Model effective field theory, Eur. Phys. J. C 75, 583 (2015).

[72] P. A. Zyla et al. (Particle Data Group), Review of particle physics, Prog. Theor. Exp. Phys. (2020), 083C01.

[73] P. Sikivie, L. Susskind, M. B. Voloshin, and V. I. Zakharov, Isospin breaking in technicolor models, Nucl. Phys. B173, 189 (1980). 
[74] CMS Collaboration, The CMS experiment at the CERN LHC, J. Instrum. 3, S08004 (2008).

[75] CMS Collaboration, Performance of the CMS Level-1 trigger in proton-proton collisions at $\sqrt{s}=13 \mathrm{TeV}$, J. Instrum. 15, P10017 (2020).

[76] CMS Collaboration, The CMS trigger system, J. Instrum. 12, P01020 (2017).

[77] CMS Collaboration, Measurements of properties of the Higgs boson decaying into the four-lepton final state in $p p$ collisions at $\sqrt{s}=13 \mathrm{TeV}, \mathrm{J}$. High Energy Phys. 11 (2017) 047.

[78] CMS Collaboration, Measurements of production cross sections of the Higgs boson in the four-lepton final state in proton-proton collisions at $\sqrt{s}=13 \mathrm{TeV}$, Eur. Phys. J. C 81, 488 (2021).

[79] CMS Collaboration, Particle-flow reconstruction and global event description with the CMS detector, J. Instrum. 12, P10003 (2017).

[80] M. Cacciari, G. P. Salam, and G. Soyez, The anti- $k_{\mathrm{T}}$ jet clustering algorithm, J. High Energy Phys. 04 (2008) 063.

[81] M. Cacciari, G. P. Salam, and G. Soyez, FastJet user manual, Eur. Phys. J. C 72, 1896 (2012).

[82] CMS Collaboration, Identification of heavy-flavour jets with the CMS detector in $p p$ collisions at $13 \mathrm{TeV}$, J. Instrum. 13, P05011 (2018).

[83] T. Sjöstrand, S. Ask, J. R. Christiansen, R. Corke, N. Desai, P. Ilten, S. Mrenna, S. Prestel, C. O. Rasmussen, and P. Z. Skands, An introduction to PYTHIA8.2, Comput. Phys. Commun. 191, 159 (2015).

[84] CMS Collaboration, Event generator tunes obtained from underlying event and multiparton scattering measurements, Eur. Phys. J. C 76, 155 (2016).

[85] CMS Collaboration, Extraction and validation of a new set of CMS PYTHIA8 tunes from underlying-event measurements, Eur. Phys. J. C 80, 4 (2020).

[86] R. D. Ball, V. Bertone, F. Cerutti, L. Del Debbio, S. Forte, A. Guffanti, J. I. Latorre, J. Rojo, and M. Ubiali (NNPDF), Unbiased global determination of parton distributions and their uncertainties at NNLO and at LO, Nucl. Phys. B855, 153 (2012).

[87] S. Agostinelli et al. (GEANT4 Collaboration), GEANT4-a simulation toolkit, Nucl. Instrum. Methods Phys. Res., Sect. A 506, 250 (2003).

[88] J. M. Campbell and R. K. Ellis, MCFM for the Tevatron and the LHC, Nucl. Phys. B, Proc. Suppl. 205-206, 10 (2010).

[89] S. Frixione, P. Nason, and C. Oleari, Matching NLO QCD computations with parton shower simulations: The POWHEG method, J. High Energy Phys. 11 (2007) 070.

[90] E. Bagnaschi, G. Degrassi, P. Slavich, and A. Vicini, Higgs production via gluon fusion in the POWHEG approach in the SM and in the MSSM, J. High Energy Phys. 02 (2012) 088.

[91] P. Nason and C. Oleari, NLO Higgs boson production via vector-boson fusion matched with shower in POWHEG, J. High Energy Phys. 02 (2010) 037.

[92] G. Luisoni, P. Nason, C. Oleari, and F. Tramontano, $H W^{ \pm} / H Z+0$ and 1 jet at NLO with the POWHEG BOX interfaced to GoSam and their merging within MiNLO, J. High Energy Phys. 10 (2013) 083.
[93] H. B. Hartanto, B. Jager, L. Reina, and D. Wackeroth, Higgs boson production in association with top quarks in the POWHEG BOX, Phys. Rev. D 91, 094003 (2015).

[94] J. Alwall, R. Frederix, S. Frixione, V. Hirschi, F. Maltoni, O. Mattelaer, H. S. Shao, T. Stelzer, P. Torrielli, and M. Zaro, The automated computation of tree-level and nextto-leading order differential cross sections, and their matching to parton shower simulations, J. High Energy Phys. 07 (2014) 079.

[95] K. Hamilton, P. Nason, and G. Zanderighi, MINLO: Multi-scale improved NLO, J. High Energy Phys. 10 (2012) 155.

[96] CMS Collaboration, A measurement of the Higgs boson mass in the diphoton decay channel, Phys. Lett. B 805, 135425 (2020).

[97] M. Grazzini, S. Kallweit, and D. Rathlev, ZZ production at the LHC: Fiducial cross sections and distributions in NNLO QCD, Phys. Lett. B 750, 407 (2015).

[98] J. M. Campbell, R. K. Ellis, and C. Williams, Vector boson pair production at the LHC, J. High Energy Phys. 07 (2011) 018.

[99] J. M. Campbell, R. K. Ellis, and C. Williams, Bounding the Higgs width at the LHC using full analytic results for $g g \rightarrow e^{-} e^{+} \mu^{-} \mu^{+}$, J. High Energy Phys. 04 (2014) 060 .

[100] J. M. Campbell and R. K. Ellis, Higgs constraints from vector boson fusion and scattering, J. High Energy Phys. 04 (2015) 030.

[101] S. Catani and M. Grazzini, An NNLO Subtraction Formalism in Hadron Collisions and Its Application to Higgs Boson Production at the LHC, Phys. Rev. Lett. 98, 222002 (2007).

[102] M. Grazzini, NNLO predictions for the Higgs boson signal in the $H \rightarrow W W \rightarrow \ell \nu \ell \nu$ and $H \rightarrow Z Z \rightarrow 4 \ell$ decay channels, J. High Energy Phys. 02 (2008) 043.

[103] M. Grazzini and H. Sargsyan, Heavy-quark mass effects in Higgs boson production at the LHC, J. High Energy Phys. 09 (2013) 129.

[104] R. J. Barlow, Extended maximum likelihood, Nucl. Instrum. Methods Phys. Res., Sect. A 297, 496 (1990).

[105] T. Chen and T.-Y. Li, Homotopy continuation method for solving systems of nonlinear and polynomial equations, Commun. Inf. Syst. 15, 119 (2015).

[106] T. Chen, T.-L. Lee, and T.-Y. Li, Hom4PS-3: A parallel numerical solver for systems of polynomial equations based on polyhedral homotopy continuation methods, in Proceedings of the Mathematical Software-ICMS 2014 (2014), p. 183, https://doi.org/10.1007/978-3-662-441992_30.

[107] T. Chen, T.-L. Lee, and T.-Y. Li, Mixed cell computation in Hom4PS-3, J. Symb. Comput. SI 79, Part 3, 516 (2017).

[108] A. S. Nemirovsky and D. B. Yudin, Problem Complexity and Method Efficiency in Optimization (Wiley, New York, 1983).

[109] Gurobi Optimization, LLC, Gurobi optimizer reference manual (2018).

[110] W. Verkerke and D. P. Kirkby, The RooFit toolkit for data modeling, in Proceedings of the 13th International 
Conference for Computing in High-Energy and Nuclear Physics (CHEP03) (2003) [eConf C0303241, MOLT007], arXiv:physics/0306116.

[111] R. Brun and F. Rademakers, ROOT: An object oriented data analysis framework, Nucl. Instrum. Methods Phys. Res., Sect. A 389, 81 (1997).

[112] S. S. Wilks, The large-sample distribution of the likelihood ratio for testing composite hypotheses, Ann. Math. Stat. 9, 60 (1938).

[113] CMS Collaboration, CMS luminosity measurements for the 2016 data taking period, CMS Physics Analysis
Summary Report No. CMS-PAS-LUM-17-001, 2017, https://cds.cern.ch/record/2257069.

[114] CMS Collaboration, CMS luminosity measurement for the 2017 data taking period at $\sqrt{s}=13 \mathrm{TeV}$, CMS Physics Analysis Summary CMS-PAS-LUM-17-004, 2018, https://cds.cern.ch/record/2621960.

[115] CMS Collaboration, CMS luminosity measurement for the 2018 data-taking period at $\sqrt{s}=13 \mathrm{TeV}$, CMS Physics Analysis Summary CMS-PAS-LUM-18-002, 2019, https://cds.cern.ch/record/2676164.

A. M. Sirunyan, ${ }^{1, a}$ A. Tumasyan, ${ }^{1}$ W. Adam, ${ }^{2}$ J. W. Andrejkovic, ${ }^{2}$ T. Bergauer, ${ }^{2}$ S. Chatterjee, ${ }^{2}$ M. Dragicevic, ${ }^{2}$ A. Escalante Del Valle, ${ }^{2}$ R. Frühwirth, ${ }^{2, b}$ M. Jeitler, ${ }^{2, b}$ N. Krammer, ${ }^{2}$ L. Lechner, ${ }^{2}$ D. Liko, ${ }^{2}$ I. Mikulec, ${ }^{2}$ P. Paulitsch, ${ }^{2}$ F. M. Pitters, ${ }^{2}$ J. Schieck, ${ }^{2, b}$ R. Schöfbeck, ${ }^{2}$ M. Spanring, ${ }^{2}$ S. Templ, ${ }^{2}$ W. Waltenberger, ${ }^{2}$ C.-E. Wulz, ${ }^{2, b}$ V. Chekhovsky, ${ }^{3}$ A. Litomin, ${ }^{3}$ V. Makarenko, ${ }^{3}$ M. R. Darwish, ${ }^{4, c}$ E. A. De Wolf, ${ }^{4}$ X. Janssen, ${ }^{4}$ T. Kello, ${ }^{4, d}$ A. Lelek, ${ }^{4}$ H. Rejeb Sfar, ${ }^{4}$ P. Van Mechelen, ${ }^{4}$ S. Van Putte, ${ }^{4}$ N. Van Remortel, ${ }^{4}$ F. Blekman, ${ }^{5}$ E. S. Bols, ${ }^{5}$ J. D'Hondt, ${ }^{5}$ J. De Clercq, ${ }^{5}$ M. Delcourt, ${ }^{5}$ H. El Faham, ${ }^{5}$ S. Lowette, ${ }^{5}$ S. Moortgat, ${ }^{5}$ A. Morton, ${ }^{5}$ D. Müller, ${ }^{5}$ A. R. Sahasransu, ${ }^{5}$ S. Tavernier, ${ }^{5}$ W. Van Doninck, ${ }^{5}$ P. Van Mulders, ${ }^{5}$ D. Beghin, ${ }^{6}$ B. Bilin, ${ }^{6}$ B. Clerbaux,${ }^{6}$ G. De Lentdecker, ${ }^{6}$ L. Favart, ${ }^{6}$ A. Grebenyuk, ${ }^{6}$ A. K. Kalsi, ${ }^{6}$ K. Lee, ${ }^{6}$ M. Mahdavikhorrami ${ }^{6}$ I. Makarenko, ${ }^{6}$ L. Moureaux, ${ }^{6}$ L. Pétré, ${ }^{6}$ A. Popov, ${ }^{6}$ N. Postiau, ${ }^{6}$ E. Starling, ${ }^{6}$ L. Thomas, ${ }^{6}$ M. Vanden Bemden, ${ }^{6}$ C. Vander Velde, ${ }^{6}$ P. Vanlaer, ${ }^{6}$ D. Vannerom, ${ }^{6}$ L. Wezenbeek, ${ }^{6}$ T. Cornelis, ${ }^{7}$ D. Dobur, ${ }^{7}$ J. Knolle, ${ }^{7}$ L. Lambrecht, ${ }^{7}$ G. Mestdach, ${ }^{7}$ M. Niedziela, ${ }^{7}$ C. Roskas, ${ }^{7}$ A. Samalan, ${ }^{7}$ K. Skovpen, ${ }^{7}$ T. T. Tran, ${ }^{7}$ M. Tytgat, ${ }^{7}$ W. Verbeke, ${ }^{7}$ B. Vermassen, ${ }^{7}$ M. Vit, ${ }^{7}$ A. Bethani, ${ }^{8}$ G. Bruno, ${ }^{8}$ F. Bury, ${ }^{8}$ C. Caputo, ${ }^{8}$ P. David, ${ }^{8}$ C. Delaere, ${ }^{8}$ I. S. Donertas, ${ }^{8}$ A. Giammanco, ${ }^{8}$ K. Jaffel, ${ }^{8}$ V. Lemaitre, ${ }^{8}$ K. Mondal,${ }^{8}$ J. Prisciandaro, ${ }^{8}$ A. Taliercio, ${ }^{8}$ M. Teklishyn, ${ }^{8}$ P. Vischia, ${ }^{8}$ S. Wertz, ${ }^{8}$ S. Wuyckens, ${ }^{8}$ G. A. Alves, ${ }^{9}$ C. Hensel,${ }^{9}$ A. Moraes, ${ }^{9}$ W. L. Aldá Júnior, ${ }^{10}$ M. Alves Gallo Pereira, ${ }^{10}$

M. Barroso Ferreira Filho, ${ }^{10}$ H. Brandao Malbouisson, ${ }^{10}$ W. Carvalho, ${ }^{10}$ J. Chinellato, ${ }^{10, \mathrm{e}}$ E. M. Da Costa, ${ }^{10}$

G. G. Da Silveira, ${ }^{10, f}$ D. De Jesus Damiao, ${ }^{10}$ S. Fonseca De Souza, ${ }^{10}$ D. Matos Figueiredo, ${ }^{10}$ C. Mora Herrera, ${ }^{10}$ K. Mota Amarilo, ${ }^{10}$ L. Mundim, ${ }^{10}$ H. Nogima, ${ }^{10}$ P. Rebello Teles, ${ }^{10}$ A. Santoro, ${ }^{10}$ S. M. Silva Do Amaral, ${ }^{10}$ A. Sznajder, ${ }^{10}$ M. Thiel, ${ }^{10}$ F. Torres Da Silva De Araujo, ${ }^{10}$ A. Vilela Pereira,${ }^{10}$ C. A. Bernardes, ${ }^{11 \mathrm{a}}$ L. Calligaris, ${ }^{11 \mathrm{a}}$

T. R. Fernandez Perez Tomei, ${ }^{11 \mathrm{a}}$ E. M. Gregores, ${ }^{11 \mathrm{a}, 11 \mathrm{~b}}$ D. S. Lemos, ${ }^{11 \mathrm{a}}$ P. G. Mercadante, ${ }^{11 \mathrm{a}, 11 \mathrm{~b}}$ S. F. Novaes, ${ }^{11 \mathrm{a}}$ Sandra S. Padula, ${ }^{11 a}$ A. Aleksandrov, ${ }^{12}$ G. Antchev, ${ }^{12}$ R. Hadjiiska, ${ }^{12}$ P. Iaydjiev, ${ }^{12}$ M. Misheva, ${ }^{12}$ M. Rodozov, ${ }^{12}$ M. Shopova, ${ }^{12}$ G. Sultanov, ${ }^{12}$ A. Dimitrov, ${ }^{13}$ T. Ivanov, ${ }^{13}$ L. Litov, ${ }^{13}$ B. Pavlov, ${ }^{13}$ P. Petkov, ${ }^{13}$ A. Petrov,${ }^{13}$ T. Cheng, ${ }^{14}$ W. Fang, ${ }^{14, d}$ Q. Guo, ${ }^{14}$ T. Javaid, ${ }^{14, g}$ M. Mittal,${ }^{14}$ H. Wang, ${ }^{14}$ L. Yuan, ${ }^{14}$ M. Ahmad, ${ }^{15}$ G. Bauer, ${ }^{15}$ C. Dozen, ${ }^{15, h}$ Z. Hu, ${ }^{15}$ J. Martins, ${ }^{15, i}$ Y. Wang, ${ }^{15}$ K. Yi, ${ }^{15, j, k}$ E. Chapon, ${ }^{16}$ G. M. Chen, ${ }^{16, g}$ H. S. Chen, ${ }^{16, g}$ M. Chen, ${ }^{16}$ F. Iemmi, ${ }^{16}$ A. Kapoor, ${ }^{16}$ D. Leggat ${ }^{16}$ H. Liao, ${ }^{16}$ Z.-A. LIU ${ }^{16,1}$ V. Milosevic, ${ }^{16}$ F. Monti, ${ }^{16}$ R. Sharma, ${ }^{16}$ J. Tao, ${ }^{16}$ J. Thomas-Wilsker, ${ }^{16}$ J. Wang, ${ }^{16}$ H. Zhang, ${ }^{16}$ S. Zhang, ${ }^{16, g}$ J. Zhao, ${ }^{16}$ A. Agapitos, ${ }^{17}$ Y. Ban, ${ }^{17}$ C. Chen, ${ }^{17}$ Q. Huang,,${ }^{17}$ A. Levin, ${ }^{17}$ Q. Li,${ }^{17}$ X. Lyu, ${ }^{17}$ Y. Mao, ${ }^{17}$ S. J. Qian, ${ }^{17}$ D. Wang, ${ }^{17}$ Q. Wang, ${ }^{17}$ J. Xiao, ${ }^{17}$ M. Lu, ${ }^{18}$ Z. You ${ }^{18}$ X. Gao, ${ }^{19, d}$ H. Okawa, ${ }^{19}$ Z. Lin, ${ }^{20}$ R. Pan,${ }^{20}$ M. Xiao, ${ }^{20}$ C. Avila, ${ }^{21}$ A. Cabrera, ${ }^{21}$ C. Florez,${ }^{21}$ J. Fraga ${ }^{21}$ A. Sarkar ${ }^{21}$ M. A. Segura Delgado, ${ }^{21}$ J. Mejia Guisao ${ }^{22}$ F. Ramirez, ${ }^{22}$ J. D. Ruiz Alvarez, ${ }^{22}$ C. A. Salazar González, ${ }^{22}$ D. Giljanovic, ${ }^{23}$ N. Godinovic, ${ }^{23}$ D. Lelas, ${ }^{23}$ I. Puljak, ${ }^{23}$ Z. Antunovic, ${ }^{24}$ M. Kovac, ${ }^{24}$ T. Sculac, ${ }^{24}$ V. Brigljevic, ${ }^{25}$ D. Ferencek, ${ }^{25}$ D. Majumder, ${ }^{25}$ M. Roguljic, ${ }^{25}$ A. Starodumov, ${ }^{25, m}$ T. Susa, ${ }^{25}$ A. Attikis, ${ }^{26}$ E. Erodotou, ${ }^{26}$ A. Ioannou, ${ }^{26}$ G. Kole, ${ }^{26}$ M. Kolosova, ${ }^{26}$ S. Konstantinou, ${ }^{26}$ J. Mousa, ${ }^{26}$ C. Nicolaou, ${ }^{26}$ F. Ptochos, ${ }^{26}$ P. A. Razis, ${ }^{26}$ H. Rykaczewski, ${ }^{26}$ H. Saka, ${ }^{26}$ M. Finger, ${ }^{27, n}$ M. Finger Jr., ${ }^{27, n}$ A. Kveton, ${ }^{27}$ E. Ayala, ${ }^{28}$ E. Carrera Jarrin, ${ }^{29}$ H. Abdalla, ${ }^{30,0}$ A. A. Abdelalim, ${ }^{30, p, q}$ A. Lotfy, ${ }^{31}$ M. A. Mahmoud, ${ }^{31}$ S. Bhowmik, ${ }^{32}$

A. Carvalho Antunes De Oliveira, ${ }^{32}$ R. K. Dewanjee, ${ }^{32}$ K. Ehataht,${ }^{32}$ M. Kadastik, ${ }^{32}$ C. Nielsen, ${ }^{32}$ J. Pata ${ }^{32}$ M. Raidal, ${ }^{32}$ L. Tani, ${ }^{32}$ C. Veelken, ${ }^{32}$ P. Eerola,${ }^{33}$ L. Forthomme, ${ }^{33}$ H. Kirschenmann, ${ }^{33}$ K. Osterberg, ${ }^{33}$ M. Voutilainen, ${ }^{33}$ S. Bharthuar, ${ }^{34}$ E. Brücken, ${ }^{34}$ F. Garcia, ${ }^{34}$ J. Havukainen,${ }^{34}$ M. S. Kim, ${ }^{34}$ R. Kinnunen, ${ }^{34}$ T. Lampén, ${ }^{34}$ K. Lassila-Perini,,${ }^{34}$ S. Lehti, ${ }^{34}$ T. Lindén, ${ }^{34}$ M. Lotti, ${ }^{34}$ L. Martikainen,,${ }^{34}$ J. Ott, ${ }^{34}$ H. Siikonen, ${ }^{34}$ E. Tuominen,${ }^{34}$ J. Tuominiemi, ${ }^{34}$ P. Luukka, ${ }^{35}$ H. Petrow, ${ }^{35}$ T. Tuuva,${ }^{35}$ C. Amendola,${ }^{36}$ M. Besancon, ${ }^{36}$ F. Couderc, ${ }^{36}$ M. Dejardin, ${ }^{36}$ D. Denegri, ${ }^{36}$ J. L. Faure,,${ }^{36}$ F. Ferri, ${ }^{36}$ S. Ganjour,${ }^{36}$ A. Givernaud, ${ }^{36}$ P. Gras, ${ }^{36}$ G. Hamel de Monchenault,${ }^{36}$ P. Jarry,${ }^{36}$ B. Lenzi,${ }^{36}$ E. Locci, ${ }^{36}$ J. Malcles, ${ }^{36}$ J. Rander, ${ }^{36}$ 
A. Rosowsky, ${ }^{36}$ M. Ö. Sahin, ${ }^{36}$ A. Savoy-Navarro,${ }^{36, r}$ M. Titov, ${ }^{36}$ G. B. Yu, ${ }^{36}$ S. Ahuja, ${ }^{37}$ F. Beaudette, ${ }^{37}$ M. Bonanomi, ${ }^{37}$ A. Buchot Perraguin, ${ }^{37}$ P. Busson, ${ }^{37}$ A. Cappati, ${ }^{37}$ C. Charlot, ${ }^{37}$ O. Davignon, ${ }^{37}$ B. Diab, ${ }^{37}$ G. Falmagne, ${ }^{37}$ S. Ghosh,${ }^{37}$ R. Granier de Cassagnac, ${ }^{37}$ A. Hakimi,${ }^{37}$ I. Kucher, ${ }^{37}$ M. Nguyen,${ }^{37}$ C. Ochando, ${ }^{37}$ P. Paganini,,${ }^{37}$ J. Rembser, ${ }^{37}$ R. Salerno, ${ }^{37}$ J. B. Sauvan, ${ }^{37}$ Y. Sirois, ${ }^{37}$ A. Zabi, ${ }^{37}$ A. Zghiche, ${ }^{37}$ J.-L. Agram, ${ }^{38, s}$ J. Andrea ${ }^{38}$ D. Apparu, ${ }^{38}$ D. Bloch, ${ }^{38}$ G. Bourgatte, ${ }^{38}$ J.-M. Brom, ${ }^{38}$ E. C. Chabert, ${ }^{38}$ C. Collard, ${ }^{38}$ D. Darej, ${ }^{38}$ J.-C. Fontaine, ${ }^{38, s}$ U. Goerlach, ${ }^{38}$ C. Grimault, ${ }^{38}$ A.-C. Le Bihan, ${ }^{38}$ E. Nibigira, ${ }^{38}$ P. Van Hove,${ }^{38}$ E. Asilar,${ }^{39}$ S. Beauceron, ${ }^{39}$ C. Bernet,${ }^{39}$ G. Boudoul,${ }^{39}$ C. Camen, ${ }^{39}$ A. Carle,${ }^{39}$ N. Chanon, ${ }^{39}$ D. Contardo ${ }^{39}$ P. Depasse, ${ }^{39}$ H. El Mamouni, ${ }^{39}$ J. Fay, ${ }^{39}$ S. Gascon, ${ }^{39}$ M. Gouzevitch, ${ }^{39}$ B. Ille, ${ }^{39}$ Sa. Jain, ${ }^{39}$ I. B. Laktineh,${ }^{39}$ H. Lattaud ${ }^{39}$ A. Lesauvage, ${ }^{39}$ M. Lethuillier, ${ }^{39}$ L. Mirabito, ${ }^{39}$ S. Perries, ${ }^{39}$ K. Shchablo, ${ }^{39}$ V. Sordini, ${ }^{39}$ L. Torterotot, ${ }^{39}$ G. Touquet, ${ }^{39}$ M. Vander Donckt, ${ }^{39}$ S. Viret, ${ }^{39}$ A. Khvedelidze, ${ }^{40, n}$ I. Lomidze ${ }^{40}$ Z. Tsamalaidze,${ }^{40, n}$ L. Feld, ${ }^{41}$ K. Klein, ${ }^{41}$ M. Lipinski, ${ }^{41}$ D. Meuser, ${ }^{41}$ A. Pauls ${ }^{41}$ M. P. Rauch, ${ }^{41}$ N. Röwert, ${ }^{41}$ J. Schulz,${ }^{41}$ M. Teroerde,${ }^{41}$ D. Eliseev,${ }^{42}$ M. Erdmann, ${ }^{42}$ P. Fackeldey, ${ }^{42}$ B. Fischer, ${ }^{42}$ S. Ghosh, ${ }^{42}$ T. Hebbeker, ${ }^{42}$ K. Hoepfner, ${ }^{42}$ F. Ivone, ${ }^{42}$ H. Keller, ${ }^{42}$ L. Mastrolorenzo, ${ }^{42}$ M. Merschmeyer, ${ }^{42}$ A. Meyer, ${ }^{42}$ G. Mocellin, ${ }^{42}$ S. Mondal, ${ }^{42}$ S. Mukherjee, ${ }^{42}$ D. Noll, ${ }^{42}$ A. Novak, ${ }^{42}$ T. Pook, ${ }^{42}$ A. Pozdnyakov, ${ }^{42}$ Y. Rath, ${ }^{42}$ H. Reithler, ${ }^{42}$ J. Roemer, ${ }^{42}$ A. Schmidt, ${ }^{42}$ S. C. Schuler, ${ }^{42}$ A. Sharma, ${ }^{42}$ S. Wiedenbeck, ${ }^{42}$ S. Zaleski, ${ }^{42}$ C. Dziwok, ${ }^{43}$ G. Flügge, ${ }^{43}$ W. Haj Ahmad, ${ }^{43, t}$ O. Hlushchenko, ${ }^{43}$ T. Kress, ${ }^{43}$ A. Nowack, ${ }^{43}$ C. Pistone ${ }^{43}$ O. Pooth, ${ }^{43}$ D. Roy, ${ }^{43}$ H. Sert, ${ }^{43}$ A. Stahl, ${ }^{43, u}$ T. Ziemons, ${ }^{43}$ H. Aarup Petersen, ${ }^{44}$ M. Aldaya Martin, ${ }^{44}$ P. Asmuss, ${ }^{44}$ I. Babounikau, ${ }^{44}$ S. Baxter, ${ }^{44}$ O. Behnke, ${ }^{44}$ A. Bermúdez Martínez, ${ }^{44}$ S. Bhattacharya, ${ }^{44}$ A. A. Bin Anuar, ${ }^{44}$ K. Borras, ${ }^{44, v}$ V. Botta, ${ }^{44}$ D. Brunner, ${ }^{44}$ A. Campbell,${ }^{44}$ A. Cardini,${ }^{44}$ C. Cheng, ${ }^{44}$ F. Colombina, ${ }^{44}$ S. Consuegra Rodríguez, ${ }^{44}$ G. Correia Silva, ${ }^{44}$ V. Danilov, ${ }^{44}$ L. Didukh, ${ }^{44}$ G. Eckerlin, ${ }^{44}$ D. Eckstein, ${ }^{44}$ L. I. Estevez Banos,${ }^{44}$ O. Filatov, ${ }^{44}$ E. Gallo, ${ }^{44, w}$ A. Geiser ${ }^{44}$ A. Giraldi, ${ }^{44}$ A. Grohsjean, ${ }^{44}$ M. Guthoff, ${ }^{44}$ A. Jafari, ${ }^{44, x}$ N. Z. Jomhari, ${ }^{44}$ H. Jung, ${ }^{44}$ A. Kasem, ${ }^{44, v}$ M. Kasemann, ${ }^{44}$ H. Kaveh, ${ }^{44}$ C. Kleinwort, ${ }^{44}$ D. Krücker, ${ }^{44}$ W. Lange, ${ }^{44}$ J. Lidrych, ${ }^{44}$ K. Lipka, ${ }^{44}$ W. Lohmann, ${ }^{44, y}$ R. Mankel, ${ }^{44}$ I.-A. Melzer-Pellmann, ${ }^{44}$ J. Metwally, ${ }^{44}$ A. B. Meyer, ${ }^{44}$ M. Meyer, ${ }^{44}$ J. Mnich, ${ }^{44}$ A. Mussgiller, ${ }^{44}$ Y. Otarid,${ }^{44}$ D. Pérez Adán, ${ }^{44}$ D. Pitzl, ${ }^{44}$ A. Raspereza,${ }^{44}$ B. Ribeiro Lopes, ${ }^{44}$ J. Rübenach,${ }^{44}$ A. Saggio, ${ }^{44}$ A. Saibel, ${ }^{44}$ M. Savitskyi,${ }^{44}$ M. Scham ${ }^{44}$ V. Scheurer ${ }^{44}$ C. Schwanenberger, ${ }^{44, w}$ A. Singh,${ }^{44}$ R. E. Sosa Ricardo, ${ }^{44}$ D. Stafford, ${ }^{44}$ N. Tonon, ${ }^{44}$ O. Turkot, ${ }^{44}$ M. Van De Klundert, ${ }^{44}$ R. Walsh, ${ }^{44}$ D. Walter, ${ }^{44}$ Y. Wen, ${ }^{44}$ K. Wichmann, ${ }^{44}$ L. Wiens, ${ }^{44}$ C. Wissing, ${ }^{44}$ S. Wuchterl, ${ }^{44}$ R. Aggleton, ${ }^{45}$ S. Bein,${ }^{45}$ L. Benato, ${ }^{45}$ A. Benecke, ${ }^{45}$ P. Connor,${ }^{45}$ K. De Leo, ${ }^{45}$ M. Eich, ${ }^{45}$ F. Feindt, ${ }^{45}$ A. Fröhlich, ${ }^{45}$ C. Garbers, ${ }^{45}$ E. Garutti, ${ }^{45}$ P. Gunnellini, ${ }^{45}$ J. Haller, ${ }^{45}$ A. Hinzmann, ${ }^{45}$ G. Kasieczka, ${ }^{45}$ R. Klanner, ${ }^{45}$

R. Kogler, ${ }^{45}$ T. Kramer, ${ }^{45}$ V. Kutzner, ${ }^{45}$ J. Lange, ${ }^{45}$ T. Lange, ${ }^{45}$ A. Lobanov, ${ }^{45}$ A. Malara, ${ }^{45}$ A. Nigamova, ${ }^{45}$

K. J. Pena Rodriguez, ${ }^{45}$ O. Rieger, ${ }^{45}$ P. Schleper, ${ }^{45}$ M. Schröder, ${ }^{45}$ J. Schwandt, ${ }^{45}$ D. Schwarz, ${ }^{45}$ J. Sonneveld, ${ }^{45}$ H. Stadie,${ }^{45}$ G. Steinbrück, ${ }^{45}$ A. Tews, ${ }^{45}$ B. Vormwald, ${ }^{45}$ I. Zoi, ${ }^{45}$ J. Bechtel,,${ }^{46}$ T. Berger, ${ }^{46}$ E. Butz, ${ }^{46}$ R. Caspart, ${ }^{46}$ T. Chwalek, ${ }^{46}$ W. De Boer, ${ }^{46, a}$ A. Dierlamm, ${ }^{46}$ A. Droll, ${ }^{46}$ K. El Morabit, ${ }^{46}$ N. Faltermann, ${ }^{46}$ M. Giffels, ${ }^{46}$ J. o. Gosewisch, ${ }^{46}$ A. Gottmann, ${ }^{46}$ F. Hartmann, ${ }^{46, u}$ C. Heidecker, ${ }^{46}$ U. Husemann, ${ }^{46}$ I. Katkov, ${ }^{46, z}$ P. Keicher, ${ }^{46}$ R. Koppenhöfer, ${ }^{46}$ S. Maier,${ }^{46}$ M. Metzler, ${ }^{46}$ S. Mitra, ${ }^{46}$ Th. Müller,${ }^{46}$ M. Neukum, ${ }^{46}$ A. Nürnberg, ${ }^{46}$ G. Quast ${ }^{46}$ K. Rabbertz, ${ }^{46}$ J. Rauser ${ }^{46}$ D. Savoiu, ${ }^{46}$ M. Schnepf,${ }^{46}$ D. Seith,${ }^{46}$ I. Shvetsov, ${ }^{46}$ H. J. Simonis, ${ }^{46}$ R. Ulrich, ${ }^{46}$ J. Van Der Linden, ${ }^{46}$ R. F. Von Cube,${ }^{46}$ M. Wassmer, ${ }^{46}$ M. Weber, ${ }^{46}$ S. Wieland, ${ }^{46}$ R. Wolf, ${ }^{46}$ S. Wozniewski, ${ }^{46}$ S. Wunsch, ${ }^{46}$ G. Anagnostou, ${ }^{47}$ P. Asenov, ${ }^{47}$ G. Daskalakis, ${ }^{47}$

T. Geralis, ${ }^{47}$ A. Kyriakis, ${ }^{47}$ D. Loukas,${ }^{47}$ A. Stakia, ${ }^{47}$ M. Diamantopoulou, ${ }^{48}$ D. Karasavvas,${ }^{48}$ G. Karathanasis, ${ }^{48}$

P. Kontaxakis, ${ }^{48}$ C. K. Koraka, ${ }^{48}$ A. Manousakis-Katsikakis, ${ }^{48}$ A. Panagiotou, ${ }^{48}$ I. Papavergou, ${ }^{48}$ N. Saoulidou, ${ }^{48}$

K. Theofilatos, ${ }^{48}$ E. Tziaferi, ${ }^{48}$ K. Vellidis, ${ }^{48}$ E. Vourliotis,${ }^{48}$ G. Bakas, ${ }^{49}$ K. Kousouris, ${ }^{49}$ I. Papakrivopoulos, ${ }^{49}$ G. Tsipolitis, ${ }^{49}$ A. Zacharopoulou, ${ }^{49}$ I. Evangelou, ${ }^{50}$ C. Foudas, ${ }^{50}$ P. Gianneios,${ }^{50}$ P. Katsoulis,${ }^{50}$ P. Kokkas, ${ }^{50}$ N. Manthos, ${ }^{50}$ I. Papadopoulos, ${ }^{50}$ J. Strologas, ${ }^{50}$ M. Csanad ${ }^{51}$ K. Farkas, ${ }^{51}$ M. M. A. Gadallah, ${ }^{51, a a}$ S. Lökös, ${ }^{51, b b}$ P. Major, ${ }^{51}$ K. Mandal,${ }^{51}$ A. Mehta, ${ }^{51}$ G. Pasztor, ${ }^{51}$ A. J. Rádl, ${ }^{51}$ O. Surányi, ${ }^{51}$ G. I. Veres, ${ }^{51}$ M. Bartók, ${ }^{52, c c}$ G. Bencze,${ }^{52}$ C. Hajdu, ${ }^{52}$ D. Horvath,${ }^{52, d d}$ F. Sikler, ${ }^{52}$ V. Veszpremi, ${ }^{52}$ G. Vesztergombi, ${ }^{52, \text { a,ee }}$ S. Czellar, ${ }^{53}$ J. Karancsi, ${ }^{53, \text { cc }}$ J. Molnar, ${ }^{53}$ Z. Szillasi, ${ }^{53}$ D. Teyssier, ${ }^{53}$ P. Raics, ${ }^{54}$ Z. L. Trocsanyi, ${ }^{54, \text { ee }}$ G. Zilizi,${ }^{54}$ T. Csorgo, ${ }^{55, f f}$ F. Nemes, ${ }^{55, f f}$ T. Novak, ${ }^{55}$ J. R. Komaragiri, ${ }^{56}$ D. Kumar, ${ }^{56}$ L. Panwar, ${ }^{56}$ P. C. Tiwari, ${ }^{56}$ S. Bahinipati, ${ }^{57, g g}$ D. Dash, ${ }^{57}$ C. Kar, ${ }^{57}$ P. Mal, ${ }^{57}$ T. Mishra,${ }^{57}$

V. K. Muraleedharan Nair Bindhu, ${ }^{57, \text { hh }}$ A. Nayak, ${ }^{57, \text { hh }}$ P. Saha, ${ }^{57}$ N. Sur ${ }^{57}$ S. K. Swain, ${ }^{57}$ D. Vats, ${ }^{57, \text { hh }}$ S. Bansal, ${ }^{58}$ S. B. Beri, ${ }^{58}$ V. Bhatnagar, ${ }^{58}$ G. Chaudhary,${ }^{58}$ S. Chauhan, ${ }^{58}$ N. Dhingra, ${ }^{58, \text { ii }}$ R. Gupta ${ }^{58}$ A. Kaur, ${ }^{58}$ M. Kaur, ${ }^{58}$ S. Kaur, ${ }^{58}$ P. Kumari, ${ }^{58}$ M. Meena, ${ }^{58}$ K. Sandeep, ${ }^{58}$ J. B. Singh ${ }^{58}$ A. K. Virdi, ${ }^{58}$ A. Ahmed, ${ }^{59}$ A. Bhardwaj, ${ }^{59}$ B. C. Choudhary, ${ }^{59}$ M. Gola, ${ }^{59}$ S. Keshri, ${ }^{59}$ A. Kumar, ${ }^{59}$ M. Naimuddin, ${ }^{59}$ P. Priyanka, ${ }^{59}$ K. Ranjan, ${ }^{59}$ A. Shah,${ }^{59}$ M. Bharti, ${ }^{60, j j}$

R. Bhattacharya, ${ }^{60} \mathrm{~S}$. Bhattacharya, ${ }^{60}$ D. Bhowmik, ${ }^{60} \mathrm{~S}$. Dutta, ${ }^{60} \mathrm{~S}$. Dutta, ${ }^{60}$ B. Gomber, ${ }^{60, k \mathrm{k}} \mathrm{M}$. Maity, ${ }^{60,11} \mathrm{~S}$. Nandan, ${ }^{60}$ P. Palit, ${ }^{60}$ P. K. Rout, ${ }^{60}$ G. Saha, ${ }^{60}$ B. Sahu, ${ }^{60}$ S. Sarkar, ${ }^{60}$ M. Sharan, ${ }^{60}$ B. Singh,${ }^{60, j j}$ S. Thakur, ${ }^{60, j j}$ P. K. Behera, ${ }^{61}$ 
S. C. Behera, ${ }^{61}$ P. Kalbhor, ${ }^{61}$ A. Muhammad, ${ }^{61}$ R. Pradhan, ${ }^{61}$ P. R. Pujahari, ${ }^{61}$ A. Sharma, ${ }^{61}$ A. K. Sikdar, ${ }^{61}$ D. Dutta, ${ }^{62}$ V. Jha, ${ }^{62}$ V. Kumar, ${ }^{62}$ D. K. Mishra, ${ }^{62}$ K. Naskar, ${ }^{62, m m}$ P. K. Netrakanti, ${ }^{62}$ L. M. Pant, ${ }^{62}$ P. Shukla, ${ }^{62}$ T. Aziz, ${ }^{63}$ S. Dugad, ${ }^{63}$ M. Kumar, ${ }^{63}$ U. Sarkar, ${ }^{63}$ S. Banerjee, ${ }^{64}$ R. Chudasama, ${ }^{64}$ M. Guchait, ${ }^{64}$ S. Karmakar, ${ }^{64}$ S. Kumar, ${ }^{64}$ G. Majumder, ${ }^{64}$ K. Mazumdar, ${ }^{64}$ S. Mukherjee, ${ }^{64}$ K. Alpana, ${ }^{65}$ S. Dube, ${ }^{65}$ B. Kansal, ${ }^{65}$ S. Pandey, ${ }^{65}$ A. Rane, ${ }^{65}$ A. Rastogi, ${ }^{65}$ S. Sharma, ${ }^{65}$ H. Bakhshiansohi, ${ }^{66, n n}$ M. Zeinali, ${ }^{66,00}$ S. Chenarani, ${ }^{67, p p}$ S. M. Etesami, ${ }^{67}$ M. Khakzad, ${ }^{67}$ M. Mohammadi Najafabadi, ${ }^{67}$ M. Grunewald, ${ }^{68}$ M. Abbrescia, ${ }^{69 a, 69 b}$ R. Aly, ${ }^{69 a, 69 b, q q}$ C. Aruta, ${ }^{69 a, 69 b}$ A. Colaleo, ${ }^{69 a}$ D. Creanza, ${ }^{69 a, 69 c}$ N. De Filippis, ${ }^{69 a, 69 c}$ M. De Palma, ${ }^{69 a, 69 b}$ A. Di Florio, ${ }^{69 a, 69 b}$ A. Di Pilato, ${ }^{69 a, 69 b}$ W. Elmetenawee, ${ }^{69 a, 69 b}$ L. Fiore, ${ }^{69 a}$ A. Gelmi, ${ }^{69 a, 69 b}$ M. Gul, ${ }^{69 a}$ G. Iaselli, ${ }^{69 a, 69 c}$ M. Ince, ${ }^{69 a, 69 b}$ S. Lezki, ${ }^{69 a, 69 b}$ G. Maggi, ${ }^{69 a, 69 c}$ M. Maggi, ${ }^{69 a}$ I. Margjeka, ${ }^{69 a, 69 b}$ V. Mastrapasqua, ${ }^{69 a, 69 b}$ J. A. Merlin, ${ }^{69 a}$ S. My, ${ }^{69 a, 69 b}$ S. Nuzzo, ${ }^{69 a, 69 b}$ A. Pellecchia, ${ }^{69 a, 69 b}$ A. Pompili, ${ }^{69 a, 69 b}$ G. Pugliese, ${ }^{69 a, 69 c}$ A. Ranieri, ${ }^{69 a}$ G. Selvaggi, ${ }^{69 a, 69 b}$ L. Silvestris, ${ }^{69 a}$ F. M. Simone, ${ }^{69 a, 69 b}$ R. Venditti, ${ }^{69 a}$ P. Verwilligen, ${ }^{69 a}$ G. Abbiendi, ${ }^{70 a}$ C. Battilana, ${ }^{70 a, 70 b}$ D. Bonacorsi, ${ }^{70 a, 70 b}$ L. Borgonovi, ${ }^{70 a}$ L. Brigliadori, ${ }^{70 a}$ R. Campanini, ${ }^{70 a, 70 b}$ P. Capiluppi, ${ }^{70 a, 70 b}$ A. Castro, ${ }^{70 a, 70 b}$

F. R. Cavallo, ${ }^{70 a}$ M. Cuffiani, ${ }^{70 a, 70 b}$ G. M. Dallavalle, ${ }^{70 a}$ T. Diotalevi, ${ }^{70 a, 70 b}$ F. Fabbri, ${ }^{70 a}$ A. Fanfani, ${ }^{70 a, 70 b}$ P. Giacomelli, ${ }^{70 a}$ L. Giommi, ${ }^{70 a, 70 b}$ C. Grandi, ${ }^{70 a}$ L. Guiducci, ${ }^{70 a, 70 b}$ S. Lo Meo, ${ }^{70 a, r r}$ L. Lunerti, ${ }^{70 a, 70 b}$ S. Marcellini, ${ }^{70 a}$ G. Masetti, ${ }^{70 a}$

F. L. Navarria, ${ }^{70 a, 70 b}$ A. Perrotta, ${ }^{70 a}$ F. Primavera, ${ }^{70 a, 70 b}$ A. M. Rossi, ${ }^{70 a, 70 b}$ T. Rovelli, ${ }^{70 a, 70 b}$ G. P. Siroli, ${ }^{70 a, 70 b}$ S. Albergo, ${ }^{71 a, 71 b, s s}$ S. Costa, ${ }^{71 a, 71 b, s s}$ A. Di Mattia, ${ }^{71 a}$ R. Potenza, ${ }^{71 a, 71 b}$ A. Tricomi, ${ }^{71 a, 71 b, s s}$ C. Tuve, ${ }^{71 a, 71 b}$ G. Barbagli, ${ }^{72 a}$ A. Cassese, ${ }^{72 a}$ R. Ceccarelli, ${ }^{72 a, 72 b}$ V. Ciulli, ${ }^{72 a, 72 b}$ C. Civinini, ${ }^{72 a}$ R. D'Alessandro, ${ }^{72 a, 72 b}$ E. Focardi, ${ }^{72 a, 72 b}$ G. Latino, ${ }^{72 a, 72 b}$ P. Lenzi, ${ }^{72 a, 72 b}$ M. Lizzo, ${ }^{72 a, 72 b}$ M. Meschini, ${ }^{72 a}$ S. Paoletti, ${ }^{72 a}$ R. Seidita, ${ }^{72 a, 72 b}$ G. Sguazzoni, ${ }^{72 a}$ L. Viliani, $^{72 a}$ L. Benussi, $^{73}$ S. Bianco, ${ }^{73}$ D. Piccolo, ${ }^{73}$ M. Bozzo, ${ }^{74 a, 74 b}$ F. Ferro, ${ }^{74 a}$ R. Mulargia, ${ }^{74 a, 74 b}$ E. Robutti, ${ }^{74 a}$ S. Tosi, ${ }^{74 a, 74 b}$ A. Benaglia, ${ }^{75 a}$ F. Brivio, ${ }^{75 a, 75 b}$ F. Cetorelli, ${ }^{75 a, 75 b}$ V. Ciriolo, ${ }^{75 a, 75 b, u}$ F. De Guio, ${ }^{75 a, 75 b}$ M. E. Dinardo, ${ }^{75 a, 75 b}$ P. Dini, ${ }^{75 a}$ S. Gennai, ${ }^{75 a}$ A. Ghezzi, ${ }^{75 a, 75 b}$ P. Govoni, ${ }^{75 a, 75 b}$ L. Guzzi, ${ }^{75 a, 75 b}$ M. Malberti, ${ }^{75 a}$ S. Malvezzi, ${ }^{75 a}$ A. Massironi, ${ }^{75 a}$ D. Menasce, ${ }^{75 a}$ L. Moroni, ${ }^{75 a}$ M. Paganoni, ${ }^{75 a, 75 b}$ D. Pedrini, ${ }^{75 a}$ S. Ragazzi, ${ }^{75 a, 75 b}$ N. Redaelli, ${ }^{75 a}$ T. Tabarelli de Fatis, ${ }^{75 a, 75 b}$ D. Valsecchi, ${ }^{75 a, 75 b, u}$ D. Zuolo, ${ }^{75 a, 75 b}$ S. Buontempo, ${ }^{76 a}$ F. Carnevali, ${ }^{76 a, 76 b}$ N. Cavallo, ${ }^{76 a, 76 c}$ A. De Iorio, ${ }^{76 a, 76 b}$ F. Fabozzi, ${ }^{76 a, 76 c}$ A. O. M. Iorio, ${ }^{76 a, 76 b}$ L. Lista, ${ }^{76 a, 76 b}$ S. Meola, ${ }^{76 a, 76 d, u}$ P. Paolucci, ${ }^{76 a, u}$ B. Rossi, ${ }^{76 a}$ C. Sciacca, ${ }^{76 a, 76 b}$ P. Azzi, ${ }^{77 a}$ N. Bacchetta, ${ }^{77 a}$ D. Bisello, ${ }^{77 a, 77 b}$ P. Bortignon, ${ }^{77 a}$ A. Bragagnolo, ${ }^{77 a, 77 b}$ R. Carlin, ${ }^{77 a, 77 b}$ P. Checchia, ${ }^{77 a}$ T. Dorigo, ${ }^{77 a}$ U. Dosselli, ${ }^{77 a}$ F. Gasparini, ${ }^{77 a, 77 b}$ U. Gasparini, ${ }^{77 a, 77 b}$ S. Y. Hoh, ${ }^{77 a, 77 b}$ L. Layer, ${ }^{77, t t}$ M. Margoni, ${ }^{77,77 b}$ A. T. Meneguzzo, ${ }^{77 a, 77 b}$ J. Pazzini, ${ }^{77 a, 77 b}$ M. Presilla, ${ }^{77 a, 77 b}$ P. Ronchese, ${ }^{77 a, 77 b}$ R. Rossin, ${ }^{77 a, 77 b}$ F. Simonetto, ${ }^{77 a, 77 b}$ G. Strong, ${ }^{77 a}$ M. Tosi, ${ }^{77 a, 77 b}$ H. YARAR, ${ }^{77 a, 77 b}$ M. Zanetti, ${ }^{77 a, 77 b}$ P. Zotto, ${ }^{77 a, 77 b}$ A. Zucchetta, ${ }^{77 a, 77 b}$ G. Zumerle, ${ }^{77 a, 77 b}$ C. Aime ${ }^{78 a, 78 b}$ A. Braghieri, ${ }^{78 a}$ S. Calzaferri, ${ }^{78 a, 78 b}$ D. Fiorina, ${ }^{78 a, 78 b}$ P. Montagna, ${ }^{78 a, 78 b}$ S. P. Ratti, ${ }^{78 a, 78 b}$ V. Re, ${ }^{78 a}$ C. Riccardi, ${ }^{78 a, 78 b}$ P. Salvini, ${ }^{78 a}$ I. Vai, ${ }^{78 a}$ P. Vitulo, ${ }^{78 a, 78 b}$ G. M. Bilei, ${ }^{79 a}$ D. Ciangottini, ${ }^{79 a, 79 b}$ L. Fanò, ${ }^{79 a, 79 b}$ P. Lariccia, ${ }^{79 a, 79 b}$ M. Magherini, ${ }^{79 a, 79 b}$ G. Mantovani, ${ }^{79 a, 79 b}$ V. Mariani, ${ }^{79 a, 79 b}$ M. Menichelli, ${ }^{79 a}$ F. Moscatelli, ${ }^{79 a}$ A. Piccinelli, ${ }^{79 a, 79 b}$ A. Rossi, ${ }^{79 a, 79 b}$ A. Santocchia, ${ }^{79 a, 79 b}$ D. Spiga, ${ }^{79 a}$ T. Tedeschi, ${ }^{79 a, 79 b}$ P. Azzurri, ${ }^{80 a}$ G. Bagliesi, ${ }^{80 a}$ V. Bertacchi, ${ }^{80 a, 80 c}$ L. Bianchini, ${ }^{80 \mathrm{a}}$ T. Boccali, ${ }^{80 \mathrm{a}}$ E. Bossini, ${ }^{80 \mathrm{a}, 80 \mathrm{~b}}$ R. Castaldi, ${ }^{80 \mathrm{a}}$ M. A. Ciocci, ${ }^{80 \mathrm{a}, 80 \mathrm{~b}}$ R. Dell'Orso, ${ }^{80 \mathrm{a}}$

M. R. Di Domenico, ${ }^{80 a, 80 d}$ S. Donato, ${ }^{80 a}$ A. Giassi, ${ }^{80 a}$ M. T. Grippo, ${ }^{80 a}$ F. Ligabue, ${ }^{80 a, 80 c}$ E. Manca, ${ }^{80 a, 80 c}$ G. Mandorli, ${ }^{80 a, 80 c}$ A. Messineo, ${ }^{80 a, 80 b}$ F. Palla, ${ }^{80 a}$ S. Parolia, ${ }^{80 a, 80 b}$ G. Ramirez-Sanchez, ${ }^{80 a, 80 c}$ A. Rizzi, ${ }^{80 a, 80 b}$ G. Rolandi, ${ }^{80 a, 80 c}$ S. Roy Chowdhury, ${ }^{80 a, 80 c}$ A. Scribano, ${ }^{80 a}$ N. Shafiei, ${ }^{80 a, 80 b}$ P. Spagnolo, ${ }^{80 a}$ R. Tenchini, ${ }^{80 a}$ G. Tonelli, ${ }^{80 a, 80 b}$ N. Turini, ${ }^{80 a, 80 d}$ A. Venturi, ${ }^{80 a}$ P. G. Verdini, ${ }^{80 a}$ M. Campana, ${ }^{81 a, 81 b}$ F. Cavallari, ${ }^{81 a}$ M. Cipriani, ${ }^{81 a, 81 b}$ D. Del Re, ${ }^{81 a, 81 b}$ E. Di Marco, ${ }^{81 a}$ M. Diemoz, ${ }^{81 a}$ E. Longo, ${ }^{81 a, 81 b}$ P. Meridiani, ${ }^{81 a}$ G. Organtini, ${ }^{81 a, 81 b}$ F. Pandolfi, ${ }^{81 a}$ R. Paramatti, ${ }^{81 a, 81 b}$ C. Quaranta, ${ }^{81 a, 81 b}$ S. Rahatlou, ${ }^{81 \mathrm{a}, 81 \mathrm{~b}}$ C. Rovelli, ${ }^{81 \mathrm{a}}$ F. Santanastasio, ${ }^{81 \mathrm{a}, 81 \mathrm{~b}}$ L. Soffi, ${ }^{81 \mathrm{a}}$ R. Tramontano, ${ }^{81 \mathrm{a}, 81 \mathrm{~b}}$ N. Amapane, ${ }^{82 \mathrm{a}, 82 \mathrm{~b}}$ R. Arcidiacono, ${ }^{82 \mathrm{a}, 82 \mathrm{c}}$ S. Argiro, ${ }^{82 \mathrm{a}, 82 \mathrm{~b}}$ M. Arneodo, ${ }^{82 \mathrm{a}, 82 \mathrm{c}}$ N. Bartosik, ${ }^{82 \mathrm{a}}$ R. Bellan, ${ }^{82 \mathrm{a}, 82 \mathrm{~b}}$ A. Bellora, ${ }^{82 \mathrm{a}, 82 \mathrm{~b}}$ J. Berenguer Antequera, ${ }^{82 a, 82 b}$ C. Biino, ${ }^{82 a}$ N. Cartiglia, ${ }^{82 a}$ S. Cometti, ${ }^{82 a}$ M. Costa, ${ }^{82 a, 82 b}$ R. Covarelli, ${ }^{82 a, 82 b}$ N. Demaria, ${ }^{82 a}$ B. Kiani, ${ }^{82 a, 82 b}$ F. Legger, ${ }^{82 a}$ C. Mariotti, ${ }^{82 a}$ S. Maselli, ${ }^{82 a}$ E. Migliore, ${ }^{82 a, 82 b}$ E. Monteil, ${ }^{82 a, 82 b}$ M. Monteno, ${ }^{82 a}$ M. M. Obertino, ${ }^{82 a, 82 b}$ G. Ortona, ${ }^{82 a}$ L. Pacher, ${ }^{82 a, 82 b}$ N. Pastrone, ${ }^{82 a}$ M. Pelliccioni, ${ }^{82 a}$ G. L. Pinna Angioni, ${ }^{82 a, 82 b}$ M. Ruspa, ${ }^{82 a, 82 c}$ R. Salvatico, ${ }^{82 a, 82 b}$ K. Shchelina, ${ }^{82 a, 82 b}$ F. Siviero, ${ }^{82 a, 82 b}$ V. Sola, ${ }^{82 a}$ A. Solano, ${ }^{82 a, 82 b}$ D. Soldi, ${ }^{82 a, 82 b}$ A. Staiano, ${ }^{82 a}$ M. Tornago, ${ }^{82 a, 82 b}$ D. Trocino, ${ }^{82 a, 82 b}$ A. Vagnerini, ${ }^{82 a}$ S. Belforte, ${ }^{83 a}$ V. Candelise, ${ }^{83 a, 83 b}$ M. Casarsa, ${ }^{83 a}$ F. Cossutti, ${ }^{83 a}$ A. Da Rold, ${ }^{83 a, 83 b}$ G. Della Ricca, ${ }^{83 a, 83 b}$ G. Sorrentino, ${ }^{83 a, 83 b}$ F. Vazzoler, ${ }^{83 a, 83 b}$ S. Dogra, ${ }^{84}$ C. Huh, ${ }^{84}$ B. Kim, ${ }^{84}$ D. H. Kim, ${ }^{84}$ G. N. Kim, ${ }^{84}$ J. Kim, ${ }^{84}$ J. Lee, ${ }^{84}$ S. W. Lee, ${ }^{84}$ C. S. Moon, ${ }^{84}$ Y. D. Oh, ${ }^{84}$ S. I. Pak, ${ }^{84}$ B. C. Radburn-Smith, ${ }^{84}$ S. Sekmen, ${ }^{84}$ Y. C. Yang, ${ }^{84}$ H. Kim, ${ }^{85}$ D. H. Moon, ${ }^{85}$ B. Francois, ${ }^{86}$ T. J. Kim, ${ }^{86}$ J. Park, ${ }^{86}$ S. Cho, ${ }^{87}$ S. Choi, ${ }^{87}$ Y. Go, ${ }^{87}$ B. Hong, ${ }^{87}$ K. Lee, ${ }^{87}$ K. S. Lee, ${ }^{87}$ J. Lim, ${ }^{87}$ J. Park, ${ }^{87}$ S. K. Park, ${ }^{87}$ J. Yoo, ${ }^{87}$ J. Goh, ${ }^{88}$ A. Gurtu, ${ }^{88}$ H. S. Kim, ${ }^{89}$ Y. Kim, ${ }^{89}$ J. Almond, ${ }^{90}$ J. H. Bhyun, ${ }^{90}$ J. Choi, ${ }^{90}$ S. Jeon, ${ }^{90}$ J. Kim, ${ }^{90}$ J. S. Kim, ${ }^{90}$ S. Ko, ${ }^{90}$ H. Kwon, ${ }^{90}$ H. Lee, ${ }^{90}$ S. Lee, ${ }^{90}$ B. H. Oh, ${ }^{90}$ 
M. Oh, ${ }^{90}$ S. B. Oh, ${ }^{90}$ H. Seo, ${ }^{90}$ U. K. Yang, ${ }^{90}$ I. Yoon,,${ }^{90}$ W. Jang, ${ }^{91}$ D. Jeon, ${ }^{91}$ D. Y. Kang, ${ }^{91}$ Y. Kang, ${ }^{91}$ J. H. Kim,,${ }^{91}$ S. Kim,${ }^{91}$ B. Ko, ${ }^{91}$ J. S. H. Lee, ${ }^{91}$ Y. Lee, ${ }^{91}$ I. C. Park, ${ }^{91}$ Y. Roh,${ }^{91}$ M. S. Ryu, ${ }^{91}$ D. Song, ${ }^{91}$ I. J. Watson, ${ }^{91}$ S. Yang, ${ }^{91}$ S. Ha, ${ }^{92}$

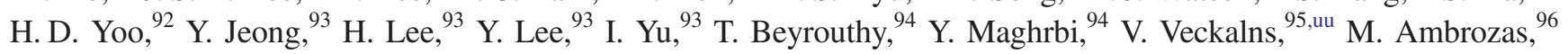
A. Juodagalvis,${ }^{96}$ A. Rinkevicius, ${ }^{96}$ G. Tamulaitis, ${ }^{96}$ A. Vaitkevicius, ${ }^{96}$ N. Bin Norjoharuddeen, ${ }^{97}$ W. A. T. Wan Abdullah, ${ }^{97}$ M. N. Yusli, ${ }^{97}$ Z. Zolkapli, ${ }^{97}$ J. F. Benitez ${ }^{98}$ A. Castaneda Hernandez, ${ }^{98}$ M. León Coello, ${ }^{98}$ J. A. Murillo Quijada, ${ }^{98}$ A. Sehrawat, ${ }^{98}$ L. Valencia Palomo, ${ }^{98}$ G. Ayala, ${ }^{99}$ H. Castilla-Valdez,${ }^{99}$ I. Heredia-De La Cruz, ${ }^{99, v v}$ R. Lopez-Fernandez,${ }^{99}$ C. A. Mondragon Herrera, ${ }^{99}$ D. A. Perez Navarro, ${ }^{99}$ A. Sanchez-Hernandez,${ }^{99}$ S. Carrillo Moreno, ${ }^{100}$ C. Oropeza Barrera, ${ }^{100}$ M. Ramirez-Garcia, ${ }^{100}$ F. Vazquez Valencia, ${ }^{100}$ I. Pedraza, ${ }^{101}$ H. A. Salazar Ibarguen, ${ }^{101}$ C. Uribe Estrada,${ }^{101}$ J. Mijuskovic, ${ }^{102, w w}$ N. Raicevic, ${ }^{102}$ D. Krofcheck, ${ }^{103}$ S. Bheesette, ${ }^{104}$ P. H. Butler, ${ }^{104}$ A. Ahmad, ${ }^{105}$ M. I. Asghar, ${ }^{105}$ A. Awais, ${ }^{105}$ M. I. M. Awan, ${ }^{105}$ H. R. Hoorani, ${ }^{105}$ W. A. Khan, ${ }^{105}$ M. A. Shah,${ }^{105}$ M. Shoaib,${ }^{105}$ M. Waqas,${ }^{105}$ V. Avati, ${ }^{106}$ L. Grzanka, ${ }^{106}$ M. Malawski, ${ }^{106}$ H. Bialkowska, ${ }^{107}$ M. Bluj, ${ }^{107}$ B. Boimska, ${ }^{107}$ M. Górski, ${ }^{107}$ M. Kazana,${ }^{107}$ M. Szleper, ${ }^{107}$ P. Zalewski, ${ }^{107}$ K. Bunkowski, ${ }^{108}$ K. Doroba ${ }^{108}$ A. Kalinowski, ${ }^{108}$ M. Konecki, ${ }^{108}$ J. Krolikowski, ${ }^{108}$ M. Walczak, ${ }^{108}$ M. Araujo, ${ }^{109}$ P. Bargassa, ${ }^{109}$ D. Bastos, ${ }^{109}$ A. Boletti, ${ }^{109}$ P. Faccioli,${ }^{109}$ M. Gallinaro, ${ }^{109}$ J. Hollar, ${ }^{109}$ N. Leonardo, ${ }^{109}$ T. Niknejad, ${ }^{109}$ M. Pisano, ${ }^{109}$ J. Seixas, ${ }^{109}$ O. Toldaiev, ${ }^{109}$ J. Varela, ${ }^{109}$ S. Afanasiev,${ }^{110}$ D. Budkouski, ${ }^{110}$ I. Golutvin,,${ }^{110}$ I. Gorbunov, ${ }^{110}$ V. Karjavine, ${ }^{110}$ V. Korenkov, ${ }^{110}$ A. Lanev, ${ }^{110}$ A. Malakhov, ${ }^{110}$ V. Matveev, ${ }^{110, x x, y y}$ V. Palichik, ${ }^{110}$ V. Perelygin, ${ }^{110}$ M. Savina, ${ }^{110}$ D. Seitova, ${ }^{110}$ V. Shalaev, ${ }^{110}$ S. Shmatov, ${ }^{110}$ S. Shulha, ${ }^{110}$ V. Smirnov, ${ }^{110}$ O. Teryaev, ${ }^{110}$ N. Voytishin, ${ }^{110}$ B.S. Yuldashev, ${ }^{110, z z}$ A. Zarubin, ${ }^{110}$ I. Zhizhin, ${ }^{110}$ G. Gavrilov, ${ }^{111}$ V. Golovtcov, ${ }^{11}$ Y. Ivanov, ${ }^{111}$ V. Kim, ${ }^{111 \text {,aaa }}$ E. Kuznetsova, ${ }^{111, \text { bbb }}$ V. Murzin, ${ }^{111}$ V. Oreshkin, ${ }^{111}$ I. Smirnov, ${ }^{111}$ D. Sosnov, ${ }^{111}$ V. Sulimov, ${ }^{111}$ L. Uvarov, ${ }^{111}$ S. Volkov, ${ }^{111}$ A. Vorobyev, ${ }^{111}$ Yu. Andreev, ${ }^{112}$ A. Dermenev, ${ }^{112}$ S. Gninenko, ${ }^{112}$ N. Golubev, ${ }^{112}$ A. Karneyeu, ${ }^{112}$ D. Kirpichnikov, ${ }^{112}$ M. Kirsanov, ${ }^{112}$ N. Krasnikov, ${ }^{112}$ A. Pashenkov, ${ }^{112}$ G. Pivovarov, ${ }^{112}$ D. Tlisov, ${ }^{112, a}$ A. Toropin, ${ }^{112}$ V. Epshteyn, ${ }^{113}$ V. Gavrilov, ${ }^{113}$ N. Lychkovskaya, ${ }^{113}$ A. Nikitenko, ${ }^{113, c c c}$ V. Popov, ${ }^{113}$ A. Spiridonov, ${ }^{113}$ A. Stepennov, ${ }^{113}$ M. Toms, ${ }^{113}$ E. Vlasov, ${ }^{113}$ A. Zhokin, ${ }^{113}$ T. Aushev, ${ }^{114}$ O. Bychkova, ${ }^{115}$ R. Chistov, ${ }^{115, \text { ddd }}$ M. Danilov, ${ }^{115 \text {,eee }}$ P. Parygin, ${ }^{115}$ S. Polikarpov, ${ }^{115 \text {,eee }}$ V. Andreev, ${ }^{116}$ M. Azarkin, ${ }^{116}$ I. Dremin, ${ }^{116}$ M. Kirakosyan, ${ }^{116}$ A. Terkulov, ${ }^{116}$ A. Belyaev, ${ }^{117}$ E. Boos ${ }^{117}$ V. Bunichev, ${ }^{117}$ M. Dubinin, ${ }^{117, f f f}$ L. Dudko, ${ }^{117}$ A. Ershov, ${ }^{117}$ V. Klyukhin, ${ }^{117}$ O. Kodolova, ${ }^{117}$ I. Lokhtin, ${ }^{117}$ S. Obraztsov, ${ }^{117}$ M. Perfilov, ${ }^{117}$ S. Petrushanko, ${ }^{117}$ V. Savrin, ${ }^{117}$ V. Blinov, ${ }^{118, g g g}$ T. Dimova, ${ }^{118, g g g}$ L. Kardapoltsev, ${ }^{118, g g g}$ A. Kozyrev, ${ }^{18, \text { ggg }}$ I. Ovtin, ${ }^{11, \text { ggg }}$ Y. Skovpen, ${ }^{118, \text { ggg }}$ I. Azhgirey, ${ }^{119}$ I. Bayshev, ${ }^{119}$ D. Elumakhov, ${ }^{119}$ V. Kachanov, ${ }^{119}$ D. Konstantinov, ${ }^{119}$ P. Mandrik, ${ }^{119}$ V. Petrov, ${ }^{119}$ R. Ryutin, ${ }^{119}$ S. Slabospitskii, ${ }^{119}$ A. Sobol,${ }^{119}$ S. Troshin,${ }^{119}$ N. Tyurin, ${ }^{119}$ A. Uzunian, ${ }^{119}$ A. Volkov, ${ }^{119}$ A. Babaev, ${ }^{120}$ V. Okhotnikov, ${ }^{120}$ V. Borchsh, ${ }^{121}$ V. Ivanchenko, ${ }^{121}$ E. Tcherniaev, ${ }^{121}$ P. Adzic, ${ }^{122, \text { hhh }}$ M. Dordevic, ${ }^{122}$ P. Milenovic, ${ }^{122} \mathrm{~J}$. Milosevic, ${ }^{122}$ M. Aguilar-Benitez, ${ }^{123} \mathrm{~J}$. Alcaraz Maestre, ${ }^{123}$ A. Álvarez Fernández, ${ }^{123}$ I. Bachiller, ${ }^{123}$ M. Barrio Luna, ${ }^{123}$ Cristina F. Bedoya, ${ }^{123}$ C. A. Carrillo Montoya,${ }^{123}$ M. Cepeda, ${ }^{123}$ M. Cerrada, ${ }^{123}$ N. Colino, ${ }^{123}$ B. De La Cruz, ${ }^{123}$ A. Delgado Peris, ${ }^{123}$ J. P. Fernández Ramos, ${ }^{123}$ J. Flix, ${ }^{123}$ M. C. Fouz, ${ }^{123}$ O. Gonzalez Lopez, ${ }^{123}$ S. Goy Lopez, ${ }^{123}$ J. M. Hernandez, ${ }^{123}$ M. I. Josa, ${ }^{123}$ J. León Holgado, ${ }^{123}$ D. Moran, ${ }^{123}$ Á. Navarro Tobar, ${ }^{123}$ A. Pérez-Calero Yzquierdo, ${ }^{123}$ J. Puerta Pelayo, ${ }^{123}$ I. Redondo, ${ }^{123}$ L. Romero, ${ }^{123}$ S. Sánchez Navas, ${ }^{123}$ L. Urda Gómez, ${ }^{123}$ C. Willmott, ${ }^{123}$ J. F. de Trocóniz, ${ }^{124}$ R. Reyes-Almanza, ${ }^{124}$ B. Alvarez Gonzalez, ${ }^{125}$ J. Cuevas, ${ }^{125}$ C. Erice, ${ }^{125}$ J. Fernandez Menendez, ${ }^{125}$ S. Folgueras, ${ }^{125}$ I. Gonzalez Caballero, ${ }^{125}$ E. Palencia Cortezon, ${ }^{125}$ C. Ramón Álvarez, ${ }^{125}$ J. Ripoll Sau, ${ }^{125}$ V. Rodríguez Bouza, ${ }^{125}$ A. Trapote, ${ }^{125}$ N. Trevisani, ${ }^{125}$ J. A. Brochero Cifuentes, ${ }^{126}$ I. J. Cabrillo, ${ }^{126}$ A. Calderon, ${ }^{126}$ J. Duarte Campderros, ${ }^{126}$ M. Fernandez, ${ }^{126}$ C. Fernandez Madrazo, ${ }^{126}$

P. J. Fernández Manteca, ${ }^{126}$ A. García Alonso, ${ }^{126}$ G. Gomez,${ }^{126}$ C. Martinez Rivero, ${ }^{126}$ P. Martinez Ruiz del Arbol, ${ }^{126}$ F. Matorras, ${ }^{126}$ P. Matorras Cuevas, ${ }^{126}$ J. Piedra Gomez, ${ }^{126}$ C. Prieels, ${ }^{126}$ T. Rodrigo, ${ }^{126}$ A. Ruiz-Jimeno, ${ }^{126}$ L. Scodellaro, ${ }^{126}$ I. Vila, ${ }^{126}$ J. M. Vizan Garcia, ${ }^{126}$ MK Jayananda, ${ }^{127}$ B. Kailasapathy, ${ }^{127, \text { iii }}$ D. U. J. Sonnadara, ${ }^{127}$

D. D. C. Wickramarathna, ${ }^{127}$ W. G. D. Dharmaratna, ${ }^{128}$ K. Liyanage, ${ }^{128}$ N. Perera, ${ }^{128}$ N. Wickramage, ${ }^{128}$ T. K. Aarrestad, ${ }^{129}$ D. Abbaneo, ${ }^{129}$ J. Alimena, ${ }^{129}$ E. Auffray, ${ }^{129}$ G. Auzinger, ${ }^{129}$ J. Baechler, ${ }^{129}$ P. Baillon, ${ }^{129, a}$ D. Barney, ${ }^{129}$ J. Bendavid, ${ }^{129}$ M. Bianco, ${ }^{129}$ A. Bocci, ${ }^{129}$ T. Camporesi, ${ }^{129}$ M. Capeans Garrido, ${ }^{129}$ G. Cerminara, ${ }^{129}$ S. S. Chhibra ${ }^{129}$ L. Cristella, ${ }^{129}$ D. d'Enterria, ${ }^{129}$ A. Dabrowski, ${ }^{129}$ N. Daci, ${ }^{129}$ A. David, ${ }^{129}$ A. De Roeck, ${ }^{129}$ M. M. Defranchis, ${ }^{129}$ M. Deile, ${ }^{129}$ M. Dobson, ${ }^{129}$ M. Dünser, ${ }^{129}$ N. Dupont, ${ }^{129}$ A. Elliott-Peisert, ${ }^{129}$ N. Emriskova, ${ }^{129}$ F. Fallavollita, ${ }^{129, j j}$ D. Fasanella, ${ }^{129}$ S. Fiorendi, ${ }^{129}$ A. Florent, ${ }^{129}$ G. Franzoni, ${ }^{129}$ W. Funk, ${ }^{129}$ S. Giani, ${ }^{129}$ D. Gigi, ${ }^{129}$ K. Gill, ${ }^{129}$ F. Glege,${ }^{129}$ L. Gouskos, ${ }^{129}$ M. Haranko, ${ }^{129}$ J. Hegeman, ${ }^{129}$ Y. Iiyama, ${ }^{129}$ V. Innocente, ${ }^{129}$ T. James, ${ }^{129}$ P. Janot, ${ }^{129}$ J. Kaspar, ${ }^{129}$ J. Kieseler, ${ }^{129}$ M. Komm, ${ }^{129}$ N. Kratochwil, ${ }^{129}$ C. Lange, ${ }^{129}$ S. Laurila, ${ }^{129}$ P. Lecoq, ${ }^{129}$ K. Long, ${ }^{129}$ C. Lourenço, ${ }^{129}$ L. Malgeri, ${ }^{129}$ S. Mallios, ${ }^{129}$ M. Mannelli, ${ }^{129}$ A. C. Marini, ${ }^{129}$ F. Meijers, ${ }^{129}$ S. Mersi, ${ }^{129}$ E. Meschi, ${ }^{129}$ F. Moortgat, ${ }^{129}$ M. Mulders, ${ }^{129}$ 
S. Orfanelli, ${ }^{129}$ L. Orsini, ${ }^{129}$ F. Pantaleo, ${ }^{129}$ L. Pape, ${ }^{129}$ E. Perez, ${ }^{129}$ M. Peruzzi, ${ }^{129}$ A. Petrilli, ${ }^{129}$ G. Petrucciani, ${ }^{129}$ A. Pfeiffer, ${ }^{129}$ M. Pierini, ${ }^{129}$ D. Piparo, ${ }^{129}$ M. Pitt, ${ }^{129}$ H. Qu, ${ }^{129}$ T. Quast, ${ }^{129}$ D. Rabady, ${ }^{129}$ A. Racz, ${ }^{129}$ G. Reales Gutiérrez, ${ }^{129}$ M. Rieger, ${ }^{129}$ M. Rovere, ${ }^{129}$ H. Sakulin, ${ }^{129}$ J. Salfeld-Nebgen, ${ }^{129}$ S. Scarfi, ${ }^{129}$ C. Schäfer, ${ }^{129}$ C. Schwick, ${ }^{129}$ M. Selvaggi, ${ }^{129}$ A. Sharma, ${ }^{129}$ P. Silva, ${ }^{129}$ W. Snoeys, ${ }^{129}$ P. Sphicas, ${ }^{129, k k k}$ S. Summers, ${ }^{129}$ V. R. Tavolaro, ${ }^{129}$ D. Treille, ${ }^{129}$ A. Tsirou, ${ }^{129}$ G. P. Van Onsem, ${ }^{129}$ M. Verzetti, ${ }^{129}$ J. Wanczyk, ${ }^{129,111}$ K. A. Wozniak, ${ }^{129}$ W. D. Zeuner, ${ }^{129}$ L. Caminada, $^{130, m m m}$ A. Ebrahimi, ${ }^{130}$ W. Erdmann, ${ }^{130}$ R. Horisberger, ${ }^{130}$ Q. Ingram, ${ }^{130}$ H. C. Kaestli, ${ }^{130}$ D. Kotlinski, ${ }^{130}$ U. Langenegger, ${ }^{130}$ M. Missiroli, ${ }^{130}$ T. Rohe, ${ }^{130}$ K. Androsov, ${ }^{131,111}$ M. Backhaus, ${ }^{131}$ P. Berger, ${ }^{131}$ A. Calandri, ${ }^{131}$ N. Chernyavskaya, ${ }^{131}$ A. De Cosa, ${ }^{131}$ G. Dissertori, ${ }^{131}$ M. Dittmar, ${ }^{131}$ M. Donegà, ${ }^{131}$ C. Dorfer, ${ }^{131}$ F. Eble, ${ }^{131}$ T. A. Gómez Espinosa, ${ }^{131}$ C. Grab, ${ }^{131}$ D. Hits, ${ }^{131}$ W. Lustermann, ${ }^{131}$ A.-M. Lyon, ${ }^{131}$ R. A. Manzoni, ${ }^{131}$ C. Martin Perez, ${ }^{131}$ M. T. Meinhard, ${ }^{131}$ F. Micheli, ${ }^{131}$ F. Nessi-Tedaldi, ${ }^{131}$ J. Niedziela, ${ }^{131}$ F. Pauss, ${ }^{131}$ V. Perovic, ${ }^{131}$ G. Perrin, ${ }^{131}$ S. Pigazzini, ${ }^{131}$ M. G. Ratti, ${ }^{131}$ M. Reichmann, ${ }^{131}$ C. Reissel, ${ }^{131}$ T. Reitenspiess, ${ }^{131}$ B. Ristic, ${ }^{131}$ D. Ruini, ${ }^{131}$ D. A. Sanz Becerra, ${ }^{131}$ M. Schönenberger, ${ }^{131}$ V. Stampf, ${ }^{131}$

J. Steggemann, ${ }^{131,111}$ R. Wallny, ${ }^{131}$ D. H. Zhu, ${ }^{131}$ C. Amsler, ${ }^{132, n n n}$ P. Bärtschi, ${ }^{132}$ C. Botta, ${ }^{132}$ D. Brzhechko, ${ }^{132}$

M. F. Canelli, ${ }^{132}$ K. Cormier, ${ }^{132}$ A. De Wit, ${ }^{132}$ R. Del Burgo, ${ }^{132}$ J. K. Heikkilä, ${ }^{132}$ M. Huwiler, ${ }^{132}$ A. Jofrehei, ${ }^{132}$ B. Kilminster, ${ }^{132}$ S. Leontsinis, ${ }^{132}$ A. Macchiolo, ${ }^{132}$ P. Meiring, ${ }^{132}$ V. M. Mikuni, ${ }^{132}$ U. Molinatti, ${ }^{132}$ I. Neutelings, ${ }^{132}$ A. Reimers, ${ }^{132}$ P. Robmann, ${ }^{132}$ S. Sanchez Cruz, ${ }^{132}$ K. Schweiger, ${ }^{132}$ Y. Takahashi, ${ }^{132}$ C. Adloff, ${ }^{133,000}$ C. M. Kuo, ${ }^{133}$ W. Lin, ${ }^{133}$ A. Roy, ${ }^{133}$ T. Sarkar, ${ }^{133,11}$ S. S. Yu, ${ }^{133}$ L. Ceard, ${ }^{134}$ Y. Chao, ${ }^{134}$ K. F. Chen, ${ }^{134}$ P. H. Chen, ${ }^{134}$ W.-S. Hou, ${ }^{134}$ Y. y. Li, ${ }^{134}$ R.-S. Lu, ${ }^{134}$ E. Paganis, ${ }^{134}$ A. Psallidas, ${ }^{134}$ A. Steen, ${ }^{134}$ H. y. Wu, ${ }^{134}$ E. Yazgan, ${ }^{134}$ P. r. Yu, ${ }^{134}$ B. Asavapibhop, ${ }^{135}$

C. Asawatangtrakuldee, ${ }^{135}$ N. Srimanobhas, ${ }^{135}$ F. Boran, ${ }^{136}$ S. Damarseckin, ${ }^{136, p p p}$ Z. S. Demiroglu, ${ }^{136}$ F. Dolek, ${ }^{136}$

I. Dumanoglu, ${ }^{136, q q q}$ E. Eskut, ${ }^{136}$ Y. Guler, ${ }^{136}$ E. Gurpinar Guler, ${ }^{136, r r r}$ I. Hos, ${ }^{136, s s s}$ C. Isik, ${ }^{136}$ O. Kara, ${ }^{136}$

A. Kayis Topaksu, ${ }^{136}$ U. Kiminsu, ${ }^{136}$ G. Onengut, ${ }^{136}$ K. Ozdemir, ${ }^{136, t t t}$ A. Polatoz, ${ }^{136}$ A. E. Simsek, ${ }^{136}$ B. Tali, ${ }^{136, \text { uuu }}$ U. G. Tok, ${ }^{136}$ S. Turkcapar, ${ }^{136}$ I. S. Zorbakir, ${ }^{136}$ C. Zorbilmez, ${ }^{136}$ B. Isildak, ${ }^{137, v v v}$ G. Karapinar, ${ }^{137, w w w}$ K. Ocalan, ${ }^{137, x x x}$ M. Yalvac, ${ }^{137, \text { yyy }}$ B. Akgun, ${ }^{138}$ I. O. Atakisi, ${ }^{138}$ E. Gülmez, ${ }^{138}$ M. Kaya, ${ }^{138, z z z}$ O. Kaya, ${ }^{138, \text { aaaa }}$ Ö. Özçelik, ${ }^{138}$ S. Tekten, ${ }^{138, b b b b}$

E. A. Yetkin, ${ }^{138, \text { cccc }}$ A. Cakir, ${ }^{139}$ K. Cankocak, ${ }^{139, \text { qqq }}$ Y. Komurcu, ${ }^{139}$ S. Sen, ${ }^{139, \text { dddd }}$ S. Cerci, ${ }^{140, \text { uuu }}$ B. Kaynak, ${ }^{140}$

S. Ozkorucuklu, ${ }^{140}$ D. Sunar Cerci, ${ }^{140, \text { uuu }}$ B. Grynyov, ${ }^{141}$ L. Levchuk, ${ }^{142}$ D. Anthony, ${ }^{143}$ E. Bhal, ${ }^{143}$ S. Bologna, ${ }^{143}$ J. J. Brooke, ${ }^{143}$ A. Bundock, ${ }^{143}$ E. Clement, ${ }^{143}$ D. Cussans, ${ }^{143}$ H. Flacher, ${ }^{143}$ J. Goldstein, ${ }^{143}$ G. P. Heath, ${ }^{143}$ H. F. Heath, ${ }^{143}$ L. Kreczko, ${ }^{143}$ B. Krikler, ${ }^{143}$ S. Paramesvaran, ${ }^{143}$ S. Seif El Nasr-Storey, ${ }^{143}$ V. J. Smith, ${ }^{143}$ N. Stylianou, ${ }^{143, \text { eeee }}$ R. White, ${ }^{143}$ K. W. Bell, ${ }^{144}$ A. Belyaev, ${ }^{144, f f f}$ C. Brew, ${ }^{144}$ R. M. Brown, ${ }^{144}$ D. J. A. Cockerill, ${ }^{144}$ K. V. Ellis, ${ }^{144}$ K. Harder, ${ }^{144}$ S. Harper, ${ }^{144}$

J. Linacre, ${ }^{144}$ K. Manolopoulos, ${ }^{144}$ D. M. Newbold, ${ }^{144}$ E. Olaiya, ${ }^{144}$ D. Petyt, ${ }^{144}$ T. Reis, ${ }^{144}$ T. Schuh, ${ }^{144}$

C. H. Shepherd-Themistocleous, ${ }^{144}$ I. R. Tomalin, ${ }^{144}$ T. Williams, ${ }^{144}$ R. Bainbridge, ${ }^{145}$ P. Bloch, ${ }^{145}$ S. Bonomally, ${ }^{145}$ J. Borg, ${ }^{145}$ S. Breeze, ${ }^{145}$ O. Buchmuller, ${ }^{145}$ V. Cepaitis, ${ }^{145}$ G. S. Chahal, ${ }^{145, g g g g}$ D. Colling, ${ }^{145}$ P. Dauncey, ${ }^{145}$ G. Davies, ${ }^{145}$

M. Della Negra, ${ }^{145}$ S. Fayer, ${ }^{145}$ G. Fedi, ${ }^{145}$ G. Hall, ${ }^{145}$ M. H. Hassanshahi, ${ }^{145}$ G. Iles, ${ }^{145}$ J. Langford, ${ }^{145}$ L. Lyons, ${ }^{145}$ A.-M. Magnan, ${ }^{145}$ S. Malik, ${ }^{145}$ A. Martelli, ${ }^{145}$ J. Nash, ${ }^{145, \text { hhhh }}$ M. Pesaresi, ${ }^{145}$ D. M. Raymond, ${ }^{145}$ A. Richards, ${ }^{145}$ A. Rose, ${ }^{145}$

E. Scott, ${ }^{145}$ C. Seez, ${ }^{145}$ A. Shtipliyski, ${ }^{145}$ A. Tapper, ${ }^{145}$ K. Uchida, ${ }^{145}$ T. Virdee, ${ }^{145, u}$ N. Wardle, ${ }^{145}$ S. N. Webb, ${ }^{145}$

D. Winterbottom, ${ }^{145}$ A. G. Zecchinelli, ${ }^{145}$ K. Coldham, ${ }^{146}$ J. E. Cole, ${ }^{146}$ A. Khan, ${ }^{146}$ P. Kyberd, ${ }^{146}$ I. D. Reid, ${ }^{146}$ L. Teodorescu, ${ }^{146}$ S. Zahid, ${ }^{146}$ S. Abdullin, ${ }^{147}$ A. Brinkerhoff, ${ }^{147}$ B. Caraway, ${ }^{147}$ J. Dittmann, ${ }^{147}$ K. Hatakeyama, ${ }^{147}$ A. R. Kanuganti, ${ }^{147}$ B. McMaster, ${ }^{147}$ N. Pastika, ${ }^{147}$ S. Sawant, ${ }^{147}$ C. Sutantawibul, ${ }^{147}$ J. Wilson, ${ }^{147}$ R. Bartek, ${ }^{148}$ A. Dominguez, ${ }^{148}$ R. Uniyal, ${ }^{148}$ A. M. Vargas Hernandez, ${ }^{148}$ A. Buccilli, ${ }^{149}$ S. I. Cooper, ${ }^{149}$ D. Di Croce, ${ }^{149}$ S. V. Gleyzer, ${ }^{149}$ C. Henderson, ${ }^{149}$ C. U. Perez, ${ }^{149}$ P. Rumerio, ${ }^{149, \text { iiii }}$ C. West, ${ }^{149}$ A. Akpinar, ${ }^{150}$ A. Albert, ${ }^{150}$ D. Arcaro, ${ }^{150}$ C. Cosby, ${ }^{150}$ Z. Demiragli, ${ }^{150}$ E. Fontanesi, ${ }^{150}$ D. Gastler, ${ }^{150}$ J. Rohlf, ${ }^{150}$ K. Salyer, ${ }^{150}$ D. Sperka, ${ }^{150}$ D. Spitzbart, ${ }^{150}$ I. Suarez, ${ }^{150}$ A. Tsatsos, ${ }^{150}$ S. Yuan, ${ }^{150}$ D. Zou, ${ }^{150}$ G. Benelli, ${ }^{151}$ B. Burkle, ${ }^{151}$ X. Coubez, ${ }^{151, v}$ D. Cutts, ${ }^{151}$ M. Hadley, ${ }^{151}$ U. Heintz, ${ }^{151}$ J. M. Hogan, ${ }^{151, j j j j}$ G. Landsberg, ${ }^{151}$ K. T. Lau, ${ }^{151}$ M. Lukasik, ${ }^{151}$ J. Luo, ${ }^{151}$ M. Narain, ${ }^{151}$ S. Sagir, ${ }^{151, k k k k}$ E. Usai, ${ }^{151}$ W. Y. Wong, ${ }^{151}$ X. Yan, ${ }^{151}$ D. Yu, ${ }^{151}$ W. Zhang, ${ }^{151}$ J. Bonilla, ${ }^{152}$ C. Brainerd, ${ }^{152}$ R. Breedon, ${ }^{152}$

M. Calderon De La Barca Sanchez, ${ }^{152}$ M. Chertok, ${ }^{152}$ J. Conway, ${ }^{152}$ P. T. Cox, ${ }^{152}$ R. Erbacher, ${ }^{152}$ G. Haza, ${ }^{152}$ F. Jensen, ${ }^{152}$ O. Kukral, ${ }^{152}$ R. Lander, ${ }^{152}$ M. Mulhearn, ${ }^{152}$ D. Pellett, ${ }^{152}$ B. Regnery, ${ }^{152}$ D. Taylor, ${ }^{152}$ Y. Yao, ${ }^{152}$ F. Zhang, ${ }^{152}$ M. Bachtis, ${ }^{153}$ R. Cousins, ${ }^{153}$ A. Datta, ${ }^{153}$ D. Hamilton, ${ }^{153}$ J. Hauser, ${ }^{153}$ M. Ignatenko, ${ }^{153}$ M. A. Iqbal, ${ }^{153}$ T. Lam, ${ }^{153}$ N. Mccoll, ${ }^{153}$ W. A. Nash, ${ }^{153}$ S. Regnard, ${ }^{153}$ D. Saltzberg, ${ }^{153}$ B. Stone, ${ }^{153}$ V. Valuev, ${ }^{153}$ K. Burt, ${ }^{154}$ Y. Chen, ${ }^{154}$ R. Clare, ${ }^{154}$ J. W. Gary, ${ }^{154}$ M. Gordon, ${ }^{154}$ G. Hanson, ${ }^{154}$ G. Karapostoli, ${ }^{154}$ O. R. Long, ${ }^{154}$ N. Manganelli, ${ }^{154}$ M. Olmedo Negrete, ${ }^{154}$ W. Si, ${ }^{154}$ S. Wimpenny, ${ }^{154}$ Y. Zhang, ${ }^{154}$ J. G. Branson, ${ }^{155}$ P. Chang, ${ }^{155}$ S. Cittolin, ${ }^{155}$ S. Cooperstein, ${ }^{155}$ N. Deelen, ${ }^{155}$ J. Duarte, ${ }^{155}$ R. Gerosa, ${ }^{155}$ L. Giannini, ${ }^{155}$ D. Gilbert, ${ }^{155}$ J. Guiang, ${ }^{155}$ R. Kansal, ${ }^{155}$ V. Krutelyov, ${ }^{155}$ R. Lee, ${ }^{155}$ J. Letts, ${ }^{155}$ 
M. Masciovecchio, ${ }^{155}$ S. May, ${ }^{155}$ M. Pieri, ${ }^{155}$ B. V. Sathia Narayanan, ${ }^{155}$ V. Sharma, ${ }^{155}$ M. Tadel, ${ }^{155}$ A. Vartak, ${ }^{155}$ F. Würthwein, ${ }^{155}$ Y. Xiang, ${ }^{155}$ A. Yagil, ${ }^{155}$ N. Amin, ${ }^{156}$ C. Campagnari, ${ }^{156}$ M. Citron, ${ }^{156}$ A. Dorsett, ${ }^{156}$ V. Dutta, ${ }^{156}$ J. Incandela, ${ }^{156}$ M. Kilpatrick, ${ }^{156}$ J. Kim, ${ }^{156}$ B. Marsh, ${ }^{156}$ H. Mei ${ }^{156}$ M. Oshiro, ${ }^{156}$ M. Quinnan, ${ }^{156}$ J. Richman, ${ }^{156}$ U. Sarica, ${ }^{156}$ D. Stuart, ${ }^{156}$ S. Wang, ${ }^{156}$ A. Bornheim, ${ }^{157}$ O. Cerri, ${ }^{157}$ I. Dutta,${ }^{157}$ J. M. Lawhorn, ${ }^{157}$ N. Lu, ${ }^{157}$ J. Mao, ${ }^{157}$ H. B. Newman, ${ }^{157}$ J. Ngadiuba, ${ }^{157}$ T. Q. Nguyen, ${ }^{157}$ M. Spiropulu, ${ }^{157}$ J. R. Vlimant, ${ }^{157}$ C. Wang, ${ }^{157}$ S. Xie,,${ }^{157}$ Z. Zhang, ${ }^{157}$ R. Y. Zhu, ${ }^{157}$ J. Alison, ${ }^{158}$ S. An, ${ }^{158}$ M. B. Andrews, ${ }^{158}$ P. Bryant, ${ }^{158}$ T. Ferguson, ${ }^{158}$ A. Harilal, ${ }^{158}$ C. Liu, ${ }^{158}$ T. Mudholkar, ${ }^{158}$ M. Paulini, ${ }^{158}$ A. Sanchez, ${ }^{158}$ J. P. Cumalat, ${ }^{159}$ W. T. Ford, ${ }^{159}$ A. Hassani,${ }^{159}$ E. MacDonald, ${ }^{159}$ R. Patel,,${ }^{159}$ A. Perloff, ${ }^{159}$ C. Savard, ${ }^{159}$ K. Stenson, ${ }^{159}$ K. A. Ulmer, ${ }^{159}$ S. R. Wagner, ${ }^{159}$ J. Alexander, ${ }^{160}$ Y. Cheng, ${ }^{160}$ D. J. Cranshaw, ${ }^{160}$ S. Hogan ${ }^{160}$ J. Monroy, ${ }^{160}$ J. R. Patterson, ${ }^{160}$ D. Quach, ${ }^{160}$ J. Reichert, ${ }^{160}$ A. Ryd, ${ }^{160}$ W. Sun, ${ }^{160}$ J. Thom, ${ }^{160}$ P. Wittich, ${ }^{160}$ R. Zou, ${ }^{160}$ M. Albrow, ${ }^{161}$ M. Alyari, ${ }^{161}$ G. Apollinari, ${ }^{161}$ A. Apresyan, ${ }^{161}$ A. Apyan, ${ }^{161}$ S. Banerjee,${ }^{161}$ L. A. T. Bauerdick, ${ }^{161}$ D. Berry, ${ }^{161}$ J. Berryhill, ${ }^{161}$ P. C. Bhat, ${ }^{161}$ K. Burkett, ${ }^{161}$ J. N. Butler, ${ }^{161}$ A. Canepa, ${ }^{161}$ G. B. Cerati, ${ }^{161}$ H. W. K. Cheung, ${ }^{161}$ F. Chlebana, ${ }^{161}$ M. Cremonesi, ${ }^{161}$ K. F. Di Petrillo, ${ }^{161}$ V. D. Elvira ${ }^{161}$ Y. Feng,${ }^{161}$ J. Freeman, ${ }^{161}$ Z. Gecse, ${ }^{161}$ L. Gray, ${ }^{161}$ D. Green, ${ }^{161}$ S. Grünendahl, ${ }^{161}$ O. Gutsche, ${ }^{161}$ R. M. Harris, ${ }^{161}$ R. Heller ${ }^{161}$ T. C. Herwig, ${ }^{161}$ J. Hirschauer, ${ }^{161}$ B. Jayatilaka, ${ }^{161}$ S. Jindariani, ${ }^{161}$ M. Johnson, ${ }^{161}$ U. Joshi, ${ }^{161}$ T. Klijnsma, ${ }^{161}$ B. Klima ${ }^{161}$ K. H. M. Kwok, ${ }^{161}$ S. Lammel, ${ }^{161}$ D. Lincoln, ${ }^{161}$ R. Lipton, ${ }^{161}$ T. Liu, ${ }^{161}$ C. Madrid ${ }^{161}$ K. Maeshima,${ }^{161}$ C. Mantilla, ${ }^{161}$ D. Mason, ${ }^{161}$ P. McBride,${ }^{161}$ P. Merkel, ${ }^{161}$ S. Mrenna, ${ }^{161}$ S. Nahn, ${ }^{161}$ V. O’Dell, ${ }^{161}$ V. Papadimitriou, ${ }^{161}$ K. Pedro, ${ }^{161}$ C. Pena, ${ }^{161, f f f}$ O. Prokofyev, ${ }^{161}$ F. Ravera, ${ }^{161}$ A. Reinsvold Hall, ${ }^{161}$ L. Ristori, ${ }^{161}$ B. Schneider, ${ }^{161}$ E. Sexton-Kennedy, ${ }^{161}$ N. Smith ${ }^{161}$ A. Soha, ${ }^{161}$ W. J. Spalding, ${ }^{161}$ L. Spiegel, ${ }^{161}$ S. Stoynev, ${ }^{161}$ J. Strait, ${ }^{161}$ L. Taylor, ${ }^{161}$ S. Tkaczyk ${ }^{161}$ N. V. Tran, ${ }^{161}$ L. Uplegger, ${ }^{161}$ E. W. Vaandering, ${ }^{161}$ H. A. Weber, ${ }^{161}$ D. Acosta, ${ }^{162}$ P. Avery, ${ }^{162}$ D. Bourilkov, ${ }^{162}$ L. Cadamuro, ${ }^{162}$ V. Cherepanov, ${ }^{162}$ F. Errico, ${ }^{162}$ R. D. Field, ${ }^{162}$ D. Guerrero, ${ }^{162}$ B. M. Joshi, ${ }^{162}$ M. Kim, ${ }^{162}$ E. Koenig, ${ }^{162}$ J. Konigsberg, ${ }^{162}$ A. Korytov, ${ }^{162}$

K. H. Lo, ${ }^{162}$ K. Matchev, ${ }^{162}$ N. Menendez, ${ }^{162}$ G. Mitselmakher, ${ }^{162}$ A. Muthirakalayil Madhu, ${ }^{162}$ N. Rawal, ${ }^{162}$

D. Rosenzweig, ${ }^{162}$ S. Rosenzweig, ${ }^{162}$ K. Shi, ${ }^{162}$ J. Sturdy, ${ }^{162}$ J. Wang ${ }^{162}$ E. Yigitbasi, ${ }^{162}$ X. Zuo, ${ }^{162}$ T. Adams, ${ }^{163}$ A. Askew, ${ }^{163}$ D. Diaz, ${ }^{163}$ R. Habibullah, ${ }^{163}$ V. Hagopian, ${ }^{163}$ K. F. Johnson, ${ }^{163}$ R. Khurana, ${ }^{163}$ T. Kolberg, ${ }^{163}$ G. Martinez, ${ }^{163}$ H. Prosper, ${ }^{163}$ C. Schiber, ${ }^{163}$ R. Yohay, ${ }^{163}$ J. Zhang, ${ }^{163}$ M. M. Baarmand, ${ }^{164}$ S. Butalla, ${ }^{164}$ T. Elkafrawy, ${ }^{164,1111}$ M. Hohlmann, ${ }^{164}$ R. Kumar Verma, ${ }^{164}$ D. Noonan, ${ }^{164}$ M. Rahmani, ${ }^{164}$ M. Saunders, ${ }^{164}$ F. Yumiceva, ${ }^{164}$ M. R. Adams, ${ }^{165}$ H. Becerril Gonzalez, ${ }^{165}$ R. Cavanaugh, ${ }^{165}$ X. Chen, ${ }^{165}$ S. Dittmer, ${ }^{165}$ O. Evdokimov, ${ }_{165}^{165}$ C. E. Gerber,${ }^{165}$ D. A. Hangal, ${ }^{165}$ D. J. Hofman, ${ }^{165}$ A. H. Merrit, ${ }^{165}$ C. Mills, ${ }^{165}$ G. Oh ${ }^{165}$ T. Roy, ${ }^{165}$ S. Rudrabhatla, ${ }^{165}$ M. B. Tonjes, ${ }^{165}$ N. Varelas, ${ }^{165}$ J. Viinikainen, ${ }^{165}$ X. Wang, ${ }^{165}$ Z. Wu, ${ }^{165}$ Z. Ye, ${ }^{165}$ M. Alhusseini, ${ }^{166}$ K. Dilsiz, ${ }^{166, m m m m}$ R. P. Gandrajula, ${ }^{166}$ O. K. Köseyan, ${ }^{166}$ J.-P. Merlo, ${ }^{166}$ A. Mestvirishvili, ${ }^{166, n n n n}$ J. Nachtman, ${ }^{166}$ H. Ogul, ${ }^{166,0000}$ Y. Onel, ${ }^{166}$ A. Penzo, ${ }^{166}$ C. Snyder, ${ }^{166}$ E. Tiras, ${ }^{166, p p p p}$ O. Amram, ${ }^{167}$ B. Blumenfeld, ${ }^{167}$ L. Corcodilos,${ }^{167}$ J. Davis, ${ }^{167}$ M. Eminizer, ${ }^{167}$ A. V. Gritsan, ${ }^{167}$ L. Kang, ${ }^{167}$ S. Kyriacou, ${ }^{167}$ P. Maksimovic, ${ }^{167}$ J. Roskes, ${ }^{167}$ M. Swartz, ${ }^{167}$ T. Á. Vámi, ${ }^{167}$ J. Anguiano, ${ }^{168}$ C. Baldenegro Barrera, ${ }^{168}$ P. Baringer, ${ }^{168}$ A. Bean ${ }^{168}$ A. Bylinkin, ${ }^{168}$ T. Isidori, ${ }^{168}$ S. Khalil ${ }^{168}$ J. King, ${ }^{168}$ G. Krintiras, ${ }^{168}$ A. Kropivnitskaya, ${ }^{168}$ C. Lindsey, ${ }^{168}$ N. Minafra, ${ }^{168}$ M. Murray, ${ }^{168}$ C. Rogan, ${ }^{168}$ C. Royon, ${ }^{168}$ S. Sanders,${ }^{168}$ E. Schmitz, ${ }^{168}$ C. Smith, ${ }^{168}$ J. D. Tapia Takaki, ${ }^{168}$ Q. Wang, ${ }^{168}$ J. Williams, ${ }^{168}$ G. Wilson, ${ }^{168}$ S. Duric, ${ }^{169}$ A. Ivanov, ${ }^{169}$ K. Kaadze, ${ }^{169}$ D. Kim, ${ }^{169}$ Y. Maravin, ${ }^{169}$ T. Mitchell, ${ }^{169}$ A. Modak, ${ }^{169} \mathrm{~K}$. Nam, ${ }^{169}$ F. Rebassoo, ${ }^{170}$ D. Wright,${ }^{170}$ E. Adams, ${ }^{171}$ A. Baden, ${ }^{171}$ O. Baron, ${ }^{171}$ A. Belloni, ${ }^{171}$ S. C. Eno, ${ }^{171}$ N. J. Hadley, ${ }^{171}$ S. Jabeen, ${ }^{171}$ R. G. Kellogg, ${ }^{171}$ T. Koeth,${ }^{171}$ A. C. Mignerey, ${ }^{171}$ S. Nabili, ${ }^{171}$ M. Seidel, ${ }^{171}$ A. Skuja, ${ }^{171}$ L. Wang, ${ }^{171}$ K. Wong, ${ }^{171}$ D. Abercrombie, ${ }^{172}$ G. Andreassi, ${ }^{172}$ R. Bi, ${ }^{172}$ S. Brandt, ${ }^{172}$ W. Busza, ${ }^{172}$ I. A. Cali, ${ }^{172}$ Y. Chen, ${ }^{172}$ M. D’Alfonso, ${ }^{172}$ J. Eysermans, ${ }^{172}$ G. Gomez Ceballos, ${ }^{172}$ M. Goncharov, ${ }^{172}$ P. Harris ${ }^{172}$ M. Hu,${ }^{172}$ M. Klute, ${ }^{172}$ D. Kovalskyi, ${ }^{172}$ J. Krupa, ${ }^{172}$ Y.-J. Lee,${ }^{172}$ B. Maier,${ }^{172}$ C. Mironov, ${ }^{172}$ C. Paus, ${ }^{172}$ D. Rankin, ${ }^{172}$ C. Roland, ${ }^{172}$ G. Roland, ${ }^{172}$ Z. Shi, ${ }^{172}$ G. S. F. Stephans, ${ }^{172}$ K. Tatar,${ }^{172}$ J. Wang, ${ }^{172}$ Z. Wang, ${ }^{172}$ B. Wyslouch, ${ }^{172}$ R. M. Chatterjee, ${ }^{173}$ A. Evans, ${ }^{173}$ P. Hansen, ${ }^{173}$ J. Hiltbrand, ${ }^{173}$ Sh. Jain, ${ }^{173}$ M. Krohn, ${ }^{173}$ Y. Kubota, ${ }^{173}$ J. Mans, ${ }^{173}$ M. Revering, ${ }^{173}$ R. Rusack, ${ }^{173}$ R. Saradhy, ${ }^{173}$ N. Schroeder, ${ }^{173}$ N. Strobbe, ${ }^{173}$ M. A. Wadud, ${ }^{173}$ K. Bloom, ${ }^{174}$ M. Bryson, ${ }^{174}$ S. Chauhan, ${ }^{174}$ D. R. Claes, ${ }^{174}$ C. Fangmeier, ${ }^{174}$ L. Finco, ${ }^{174}$ F. Golf, ${ }^{174}$ J. R. González Fernández, ${ }^{174}$ C. Joo, ${ }^{174}$ I. Kravchenko, ${ }^{174}$ M. Musich,${ }^{174}$ I. Reed,${ }^{174}$ J. E. Siado,${ }^{174}$ G. R. Snow, ${ }^{174, a}$ W. Tabb,${ }^{174}$ F. Yan, ${ }^{174}$ G. Agarwal, ${ }^{175}$

H. Bandyopadhyay, ${ }^{175}$ L. Hay, ${ }^{175}$ I. Iashvili, ${ }^{175}$ A. Kharchilava, ${ }^{175}$ C. McLean, ${ }^{175}$ D. Nguyen, ${ }^{175}$ J. Pekkanen, ${ }^{175}$ S. Rappoccio, ${ }^{175}$ A. Williams, ${ }^{175}$ G. Alverson, ${ }^{176}$ E. Barberis, ${ }^{176}$ C. Freer, ${ }^{176}$ Y. Haddad, ${ }^{176}$ A. Hortiangtham, ${ }^{176}$ J. Li, ${ }^{176}$ G. Madigan, ${ }^{176}$ B. Marzocchi, ${ }^{176}$ D. M. Morse,${ }^{176}$ V. Nguyen, ${ }^{176}$ T. Orimoto ${ }^{176}$ A. Parker, ${ }^{176}$ L. Skinnari, ${ }^{176}$ A. Tishelman-Charny, ${ }^{176}$ T. Wamorkar, ${ }^{176}$ B. Wang, ${ }^{176}$ A. Wisecarver, ${ }^{176}$ D. Wood, ${ }^{176}$ S. Bhattacharya,${ }^{177}$ J. Bueghly, ${ }^{177}$ Z. Chen, ${ }^{177}$ A. Gilbert, ${ }^{177}$ T. Gunter, ${ }^{177}$ K. A. Hahn, ${ }^{177}$ N. Odell, ${ }^{177}$ M. H. Schmitt, ${ }^{177}$ M. Velasco, ${ }^{177}$ R. Band, ${ }^{178}$ R. Bucci, ${ }^{178}$ 
A. Das, ${ }^{178}$ N. Dev, ${ }^{178}$ R. Goldouzian, ${ }^{178}$ M. Hildreth, ${ }^{178}$ K. Hurtado Anampa,${ }^{178}$ C. Jessop,${ }^{178}$ K. Lannon, ${ }^{178}$ N. Loukas, ${ }^{178}$ N. Marinelli, ${ }^{178}$ I. Mcalister, ${ }^{178}$ T. McCauley, ${ }^{178}$ F. Meng, ${ }^{178}$ K. Mohrman, ${ }^{178}$ Y. Musienko, ${ }^{178, x x}$ R. Ruchti, ${ }^{178}$ P. Siddireddy, ${ }^{178}$ M. Wayne, ${ }^{178}$ A. Wightman, ${ }^{178}$ M. Wolf, ${ }^{178}$ M. Zarucki, ${ }^{178}$ L. Zygala, ${ }^{178}$ B. Bylsma, ${ }^{179}$ B. Cardwell, ${ }^{179}$ L. S. Durkin, ${ }^{179}$ B. Francis, ${ }^{179}$ C. Hill, ${ }^{179}$ M. Nunez Ornelas, ${ }^{179}$ K. Wei, ${ }^{179}$ B. L. Winer, ${ }^{179}$ B. R. Yates, ${ }^{179}$ F. M. Addesa, ${ }^{180}$ B. Bonham, ${ }^{180}$ P. Das, ${ }^{180}$ G. Dezoort, ${ }^{180}$ P. Elmer, ${ }^{180}$ A. Frankenthal, ${ }^{180}$ B. Greenberg, ${ }^{180}$ N. Haubrich, ${ }^{180}$

S. Higginbotham, ${ }^{180}$ A. Kalogeropoulos, ${ }^{180}$ G. Kopp,${ }^{180}$ S. Kwan, ${ }^{180}$ D. Lange,${ }^{180}$ M. T. Lucchini, ${ }^{180}$ D. Marlow, ${ }^{180}$ K. Mei, ${ }^{180}$ I. Ojalvo, ${ }^{180}$ J. Olsen, ${ }^{180}$ C. Palmer, ${ }^{180}$ D. Stickland, ${ }^{180}$ C. Tully, ${ }^{180}$ S. Malik, ${ }^{181}$ S. Norberg, ${ }^{181}$ A. S. Bakshi, ${ }^{182}$ V. E. Barnes, ${ }^{182}$ R. Chawla, ${ }^{182}$ S. Das, ${ }^{182}$ L. Gutay, ${ }^{182}$ M. Jones, ${ }^{182}$ A. W. Jung, ${ }^{182}$ S. Karmarkar, ${ }^{182}$ M. Liu, ${ }^{182}$ G. Negro, ${ }^{182}$ N. Neumeister, ${ }^{182}$ G. Paspalaki, ${ }^{182}$ C. C. Peng ${ }^{182}$ S. Piperov, ${ }^{182}$ A. Purohit ${ }^{182}$ J. F. Schulte, ${ }^{182}$ M. Stojanovic, ${ }^{182, r}$ J. Thieman, ${ }^{182}$ F. Wang, ${ }^{182}$ R. Xiao, ${ }^{182}$ W. Xie, ${ }^{182}$ J. Dolen, ${ }^{183}$ N. Parashar, ${ }^{183}$ A. Baty, ${ }^{184}$ M. Decaro, ${ }^{184}$ S. Dildick, ${ }^{184}$ K. M. Ecklund, ${ }^{184}$ S. Freed, ${ }^{184}$ P. Gardner, ${ }^{184}$ F. J. M. Geurts, ${ }^{184}$ A. Kumar, ${ }^{184}$ W. Li ${ }^{184}$ B. P. Padley, ${ }^{184}$ R. Redjimi, ${ }^{184}$ W. Shi, ${ }^{184}$ A. G. Stahl Leiton, ${ }^{184}$ S. Yang, ${ }^{184}$ L. Zhang, ${ }^{184}$ Y. Zhang, ${ }^{184}$ A. Bodek,${ }^{185}$ P. de Barbaro, ${ }^{185}$ R. Demina, ${ }^{185}$ J. L. Dulemba, ${ }^{185}$ C. Fallon, ${ }^{185}$ T. Ferbel,${ }^{185}$ M. Galanti, ${ }^{185}$ A. Garcia-Bellido, ${ }^{185}$ O. Hindrichs, ${ }^{185}$ A. Khukhunaishvili, ${ }^{185}$ E. Ranken, ${ }^{185}$ R. Taus, ${ }^{185}$ B. Chiarito, ${ }^{186}$ J. P. Chou, ${ }^{186}$ A. Gandrakota, ${ }^{186}$ Y. Gershtein, ${ }^{186}$ E. Halkiadakis, ${ }^{186}$ A. Hart, ${ }^{186}$ M. Heindl, ${ }^{186}$ E. Hughes, ${ }^{186}$ S. Kaplan, ${ }^{186}$ O. Karacheban, ${ }^{186, y}$ I. Laflotte, ${ }^{186}$ A. Lath ${ }^{186}$ R. Montalvo, ${ }^{186}$ K. Nash, ${ }^{186}$ M. Osherson, ${ }^{186}$ S. Salur, ${ }^{186}$ S. Schnetzer, ${ }^{186}$ S. Somalwar, ${ }^{186}$ R. Stone, ${ }^{186}$ S. A. Thayil, ${ }^{186}$ S. Thomas, ${ }^{186}$ H. Wang, ${ }^{186}$ H. Acharya, ${ }^{187}$ A. G. Delannoy, ${ }^{187}$ S. Spanier, ${ }^{187}$ O. Bouhali, ${ }^{188, q q q q}$ M. Dalchenko, ${ }^{188}$ A. Delgado, ${ }^{188}$ R. Eusebi, ${ }^{188}$ J. Gilmore, ${ }^{188}$ T. Huang, ${ }^{188}$ T. Kamon, ${ }^{188, r r r r}$ H. Kim, ${ }^{188}$ S. Luo, ${ }^{188}$ S. Malhotra, ${ }^{188}$ R. Mueller, ${ }^{188}$ D. Overton, ${ }^{188}$ D. Rathjens, ${ }^{188}$ A. Safonov, ${ }^{188}$ N. Akchurin, ${ }^{189}$ J. Damgov, ${ }^{189}$ V. Hegde, ${ }^{189}$ S. Kunori, ${ }^{189}$ K. Lamichhane, ${ }^{189}$ S. W. Lee, ${ }^{189}$ T. Mengke, ${ }^{189}$ S. Muthumuni, ${ }^{189}$ T. Peltola, ${ }^{189}$ I. Volobouev, ${ }^{189}$ Z. Wang, ${ }^{189}$ A. Whitbeck, ${ }^{189}$ E. Appelt, ${ }^{190}$ S. Greene, ${ }^{190}$ A. Gurrola, ${ }^{190}$ W. Johns, ${ }^{190}$ A. Melo, ${ }^{190}$ H. Ni, ${ }^{190}$ K. Padeken, ${ }^{190}$ F. Romeo, ${ }^{190}$ P. Sheldon, ${ }^{190}$ S. Tuo, ${ }^{190}$ J. Velkovska, ${ }^{190}$ M. W. Arenton, ${ }^{191}$ B. Cox,${ }^{191}$ G. Cummings,${ }^{191}$ J. Hakala, ${ }^{191}$ R. Hirosky, ${ }^{191}$ M. Joyce ${ }^{191}$ A. Ledovskoy, ${ }^{191}$ A. Li ${ }^{191}$ C. Neu, ${ }^{191}$ B. Tannenwald,${ }^{191}$ S. White, ${ }^{191}$ E. Wolfe,${ }^{191}$ N. Poudyal, ${ }^{192}$ K. Black,${ }^{193}$ T. Bose, ${ }^{193}$ J. Buchanan,${ }^{193}$ C. Caillol, ${ }^{193}$ S. Dasu, ${ }^{193}$ I. De Bruyn, ${ }^{193}$ P. Everaerts, ${ }^{193}$ F. Fienga, ${ }^{193}$ C. Galloni, ${ }^{193}$ H. He ${ }^{193}$ M. Herndon, ${ }^{193}$ A. Hervé ${ }^{193}$ U. Hussain, ${ }^{193}$ A. Lanaro, ${ }^{193}$ A. Loeliger ${ }^{193}$ R. Loveless, ${ }^{193}$ J. Madhusudanan Sreekala, ${ }^{193}$ A. Mallampalli, ${ }^{193}$ A. Mohammadi, ${ }^{193}$ D. Pinna, ${ }^{193}$ A. Savin, ${ }^{193}$ V. Shang, ${ }^{193}$ V. Sharma, ${ }^{193}$ W. H. Smith, ${ }^{193}$ D. Teague, ${ }^{193}$ S. Trembath-Reichert, ${ }^{193}$ and W. Vetens ${ }^{193}$

(CMS Collaboration)

\author{
${ }^{1}$ Yerevan Physics Institute, Yerevan, Armenia \\ ${ }^{2}$ Institut für Hochenergiephysik, Wien, Austria \\ ${ }^{3}$ Institute for Nuclear Problems, Minsk, Belarus \\ ${ }^{4}$ Universiteit Antwerpen, Antwerpen, Belgium \\ ${ }^{5}$ Vrije Universiteit Brussel, Brussel, Belgium \\ ${ }^{6}$ Université Libre de Bruxelles, Bruxelles, Belgium \\ ${ }^{7}$ Ghent University, Ghent, Belgium \\ ${ }^{8}$ Université Catholique de Louvain, Louvain-la-Neuve, Belgium \\ ${ }^{9}$ Centro Brasileiro de Pesquisas Fisicas, Rio de Janeiro, Brazil \\ ${ }^{10}$ Universidade do Estado do Rio de Janeiro, Rio de Janeiro, Brazil \\ ${ }^{11 a}$ Universidade Estadual Paulista, São Paulo, Brazil \\ ${ }^{11 \mathrm{~b}}$ Universidade Federal do ABC, São Paulo, Brazil \\ ${ }^{12}$ Institute for Nuclear Research and Nuclear Energy, Bulgarian Academy of Sciences, Sofia, Bulgaria \\ ${ }^{13}$ University of Sofia, Sofia, Bulgaria \\ ${ }^{14}$ Beihang University, Beijing, China \\ ${ }^{15}$ Department of Physics, Tsinghua University, Beijing, China \\ ${ }^{16}$ Institute of High Energy Physics, Beijing, China \\ ${ }^{17}$ State Key Laboratory of Nuclear Physics and Technology, Peking University, Beijing, China \\ ${ }^{18}$ Sun Yat-Sen University, Guangzhou, China \\ ${ }^{19}$ Institute of Modern Physics and Key Laboratory of Nuclear Physics and Ion-beam Application (MOE) - \\ Fudan University, Shanghai, China \\ ${ }^{20}$ Zhejiang University, Hangzhou, China \\ ${ }^{21}$ Universidad de Los Andes, Bogota, Colombia
}




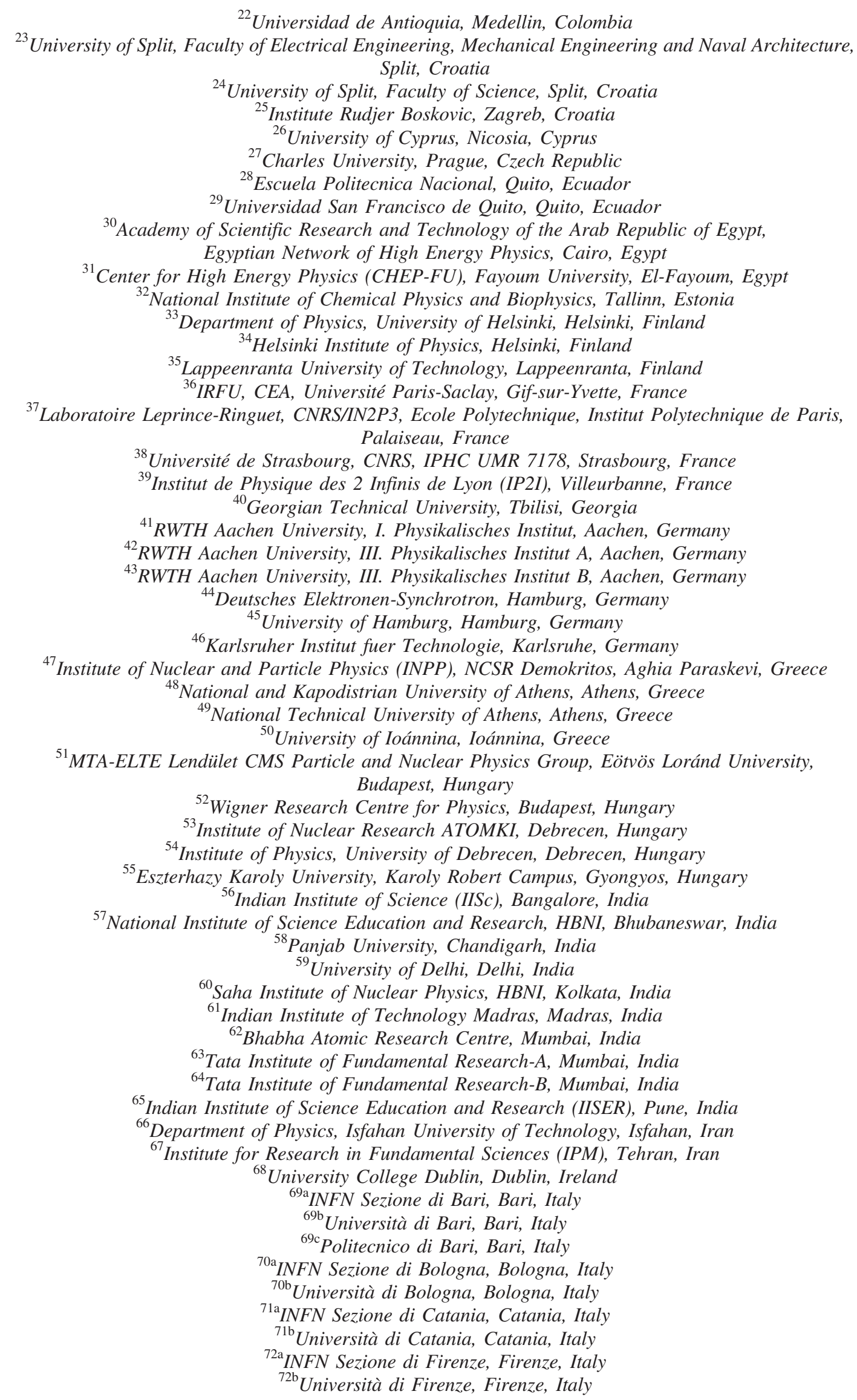


${ }^{73}$ INFN Laboratori Nazionali di Frascati, Frascati, Italy

${ }^{74 a}$ INFN Sezione di Genova, Genova, Italy

${ }^{74 \mathrm{~b}}$ Università di Genova, Genova, Italy

${ }^{75 a}$ INFN Sezione di Milano-Bicocca, Milano, Italy

${ }^{75 b}$ Università di Milano-Bicocca, Milano, Italy

${ }^{76 a}$ INFN Sezione di Napoli, Napoli, Italy

${ }^{76 \mathrm{~b}}$ Università di Napoli 'Federico II', Napoli, Italy

${ }^{76 c}$ Università della Basilicata, Roma, Italy

${ }^{76 \mathrm{~d}}$ Università G. Marconi, Roma, Italy

${ }^{77 \mathrm{a}}$ INFN Sezione di Padova, Padova, Italy

${ }^{77 b}$ Università di Padova, Padova, Italy

${ }^{77 \mathrm{c}}$ Università di Trento, Trento, Italy

${ }^{78 a}$ INFN Sezione di Pavia, Pavia, Italy

${ }^{78 b}$ Università di Pavia, Pavia, Italy

${ }^{79 a}$ INFN Sezione di Perugia, Perugia, Italy

${ }^{79 b}$ Università di Perugia, Perugia, Italy

${ }^{80 a}$ INFN Sezione di Pisa, Pisa Italy

${ }^{80 \mathrm{~b}}$ Università di Pisa, Pisa Italy

${ }^{80 \mathrm{c}}$ Scuola Normale Superiore di Pisa, Pisa Italy

${ }^{80 \mathrm{~d}}$ Università di Siena, Siena, Italy

${ }^{81 a}$ INFN Sezione di Roma, Rome, Italy

${ }^{81 \mathrm{~b}}$ Sapienza Università di Roma, Rome, Italy

${ }^{82 a}$ INFN Sezione di Torino, Torino, Italy

${ }^{82 \mathrm{~b}}$ Università di Torino, Torino, Italy

${ }^{82 \mathrm{c}}$ Università del Piemonte Orientale, Novara, Italy

${ }^{83 a}$ INFN Sezione di Trieste, Trieste, Italy

${ }^{83 \mathrm{~b}}$ Università di Trieste, Trieste, Italy

${ }^{84}$ Kyungpook National University, Daegu, Korea

${ }^{85}$ Chonnam National University, Institute for Universe and Elementary Particles, Kwangju, Korea

${ }^{86}$ Hanyang University, Seoul, Korea

${ }^{87}$ Korea University, Seoul, Korea

${ }^{88}$ Kyung Hee University, Department of Physics, Seoul, Republic of Korea

${ }^{89}$ Sejong University, Seoul, Korea

${ }^{90}$ Seoul National University, Seoul, Korea

${ }^{91}$ University of Seoul, Seoul, Korea

${ }^{92}$ Yonsei University, Department of Physics, Seoul, Korea

${ }^{93}$ Sungkyunkwan University, Suwon, Korea

${ }^{94}$ College of Engineering and Technology, American University of the Middle East (AUM), Egaila, Kuwait

${ }^{95}$ Riga Technical University, Riga, Latvia

${ }^{96}$ Vilnius University, Vilnius, Lithuania

${ }^{97}$ National Centre for Particle Physics, Universiti Malaya, Kuala Lumpur, Malaysia

${ }^{98}$ Universidad de Sonora (UNISON), Hermosillo, Mexico

${ }^{99}$ Centro de Investigacion y de Estudios Avanzados del IPN, Mexico City, Mexico

${ }^{100}$ Universidad Iberoamericana, Mexico City, Mexico

${ }^{101}$ Benemerita Universidad Autonoma de Puebla, Puebla, Mexico

${ }^{102}$ University of Montenegro, Podgorica, Montenegro

${ }^{103}$ University of Auckland, Auckland, New Zealand

${ }^{104}$ University of Canterbury, Christchurch, New Zealand

${ }^{105}$ National Centre for Physics, Quaid-I-Azam University, Islamabad, Pakistan

${ }^{106}$ AGH University of Science and Technology Faculty of Computer Science,

Electronics and Telecommunications, Krakow, Poland

${ }^{107}$ National Centre for Nuclear Research, Swierk, Poland

${ }^{108}$ Institute of Experimental Physics, Faculty of Physics, University of Warsaw, Warsaw, Poland

${ }^{109}$ Laboratório de Instrumentação e Física Experimental de Partículas, Lisboa, Portugal

${ }^{110}$ Joint Institute for Nuclear Research, Dubna, Russia

${ }^{111}$ Petersburg Nuclear Physics Institute, Gatchina (St. Petersburg), Russia

${ }^{112}$ Institute for Nuclear Research, Moscow, Russia

${ }^{113}$ Institute for Theoretical and Experimental Physics named by A.I. Alikhanov of NRC 'Kurchatov Institute', Moscow, Russia

${ }^{114}$ Moscow Institute of Physics and Technology, Moscow, Russia 


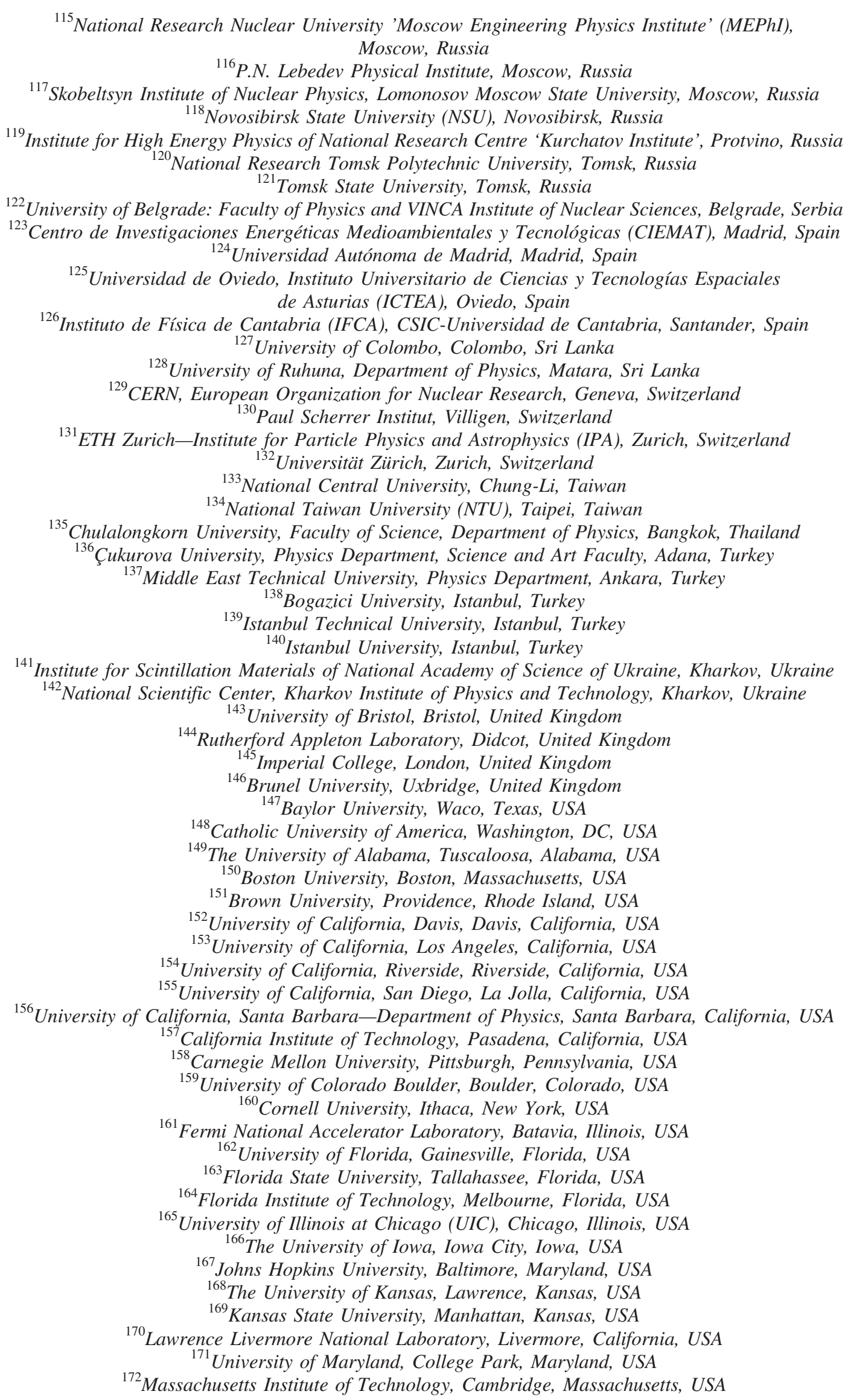




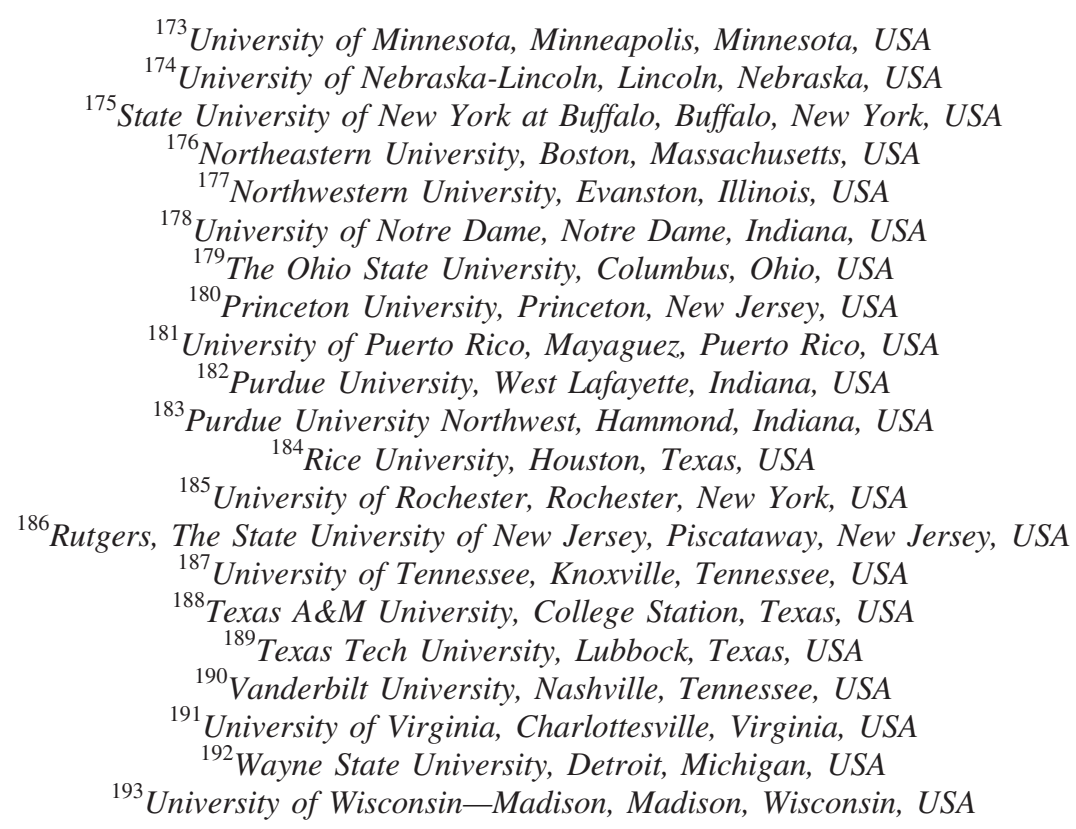

${ }^{\mathrm{a}}$ Deceased.

${ }^{\mathrm{b}}$ Also at TU Wien, Vienna, Austria.

${ }^{\mathrm{c}}$ Also at Institute of Basic and Applied Sciences, Faculty of Engineering, Arab Academy for Science, Technology and Maritime Transport, Alexandria, Egypt.

${ }^{\mathrm{d}}$ Also at Université Libre de Bruxelles, Bruxelles, Belgium.

${ }^{\mathrm{e}}$ Also at Universidade Estadual de Campinas, Campinas, Brazil.

${ }_{\mathrm{f}}^{\mathrm{f}}$ Also at Federal University of Rio Grande do Sul, Porto Alegre, Brazil.

${ }^{g}$ Also at University of Chinese Academy of Sciences, Beijing, China.

${ }^{\mathrm{h}}$ Also at Department of Physics, Tsinghua University, Beijing, China.

${ }^{i}$ Also at Universidade Federal do Mato Grosso do Sul (UFMS), Nova Andradina, Mato Grosso do Sul, Brazil.

${ }^{\mathrm{j}}$ Also at The University of Iowa, Iowa City, Iowa, USA.

${ }^{\mathrm{k}}$ Also at Nanjing Normal University, Department of Physics.

${ }^{1}$ Also at University of Chinese Academy of Sciences, Beijing, China.

${ }^{\mathrm{m}}$ Also at Institute for Theoretical and Experimental Physics named by A.I. Alikhanov of NRC 'Kurchatov Institute', Moscow, Russia.

${ }^{\mathrm{n}}$ Also at Joint Institute for Nuclear Research, Dubna, Russia.

${ }^{\circ}$ Also at Cairo University, Cairo, Egypt.

${ }^{\mathrm{p}}$ Also at Helwan University, Cairo, Egypt.

${ }^{\mathrm{q}}$ Also at Zewail City of Science and Technology, Zewail, Egypt.

${ }^{\mathrm{r}}$ Also at Purdue University, West Lafayette, Indiana, USA.

${ }^{\mathrm{s}}$ Also at Université de Haute Alsace, Mulhouse, France.

${ }^{\mathrm{t}}$ Also at Erzincan Binali Yildirim University, Erzincan, Turkey.

"Also at CERN, European Organization for Nuclear Research, Geneva, Switzerland.

${ }^{v}$ Also at RWTH Aachen University, III. Physikalisches Institut A, Aachen, Germany.

${ }^{w}$ Also at University of Hamburg, Hamburg, Germany.

${ }^{\mathrm{x}}$ Also at Department of Physics, Isfahan University of Technology, Isfahan, Iran.

${ }^{y}$ Also at Brandenburg University of Technology, Cottbus, Germany.

${ }^{\mathrm{z}}$ Also at Skobeltsyn Institute of Nuclear Physics, Lomonosov Moscow State University, Moscow, Russia.

${ }^{\text {aa } A l s o ~ a t ~ P h y s i c s ~ D e p a r t m e n t, ~ F a c u l t y ~ o f ~ S c i e n c e, ~ A s s i u t ~ U n i v e r s i t y . ~}$

${ }^{\mathrm{bb}}$ Also at Eszterhazy Karoly University, Karoly Robert Campus, Gyongyos, Hungary.

${ }^{c c}$ Also at Institute of Physics, University of Debrecen, Debrecen, Hungary.

${ }^{\mathrm{dd}}$ Also at Institute of Nuclear Research ATOMKI, Debrecen, Hungary.

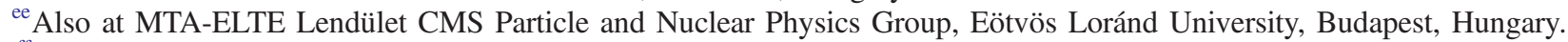

${ }^{\mathrm{ff}}$ Also at Wigner Research Centre for Physics, Budapest, Hungary.

${ }^{\mathrm{gg}}$ Also at IIT Bhubaneswar, Bhubaneswar, India.

${ }^{\text {hh }}$ Also at Institute of Physics, Bhubaneswar, India.

${ }^{\text {ii }}$ Also at G. H. G. Khalsa College, Punjab, India.

${ }^{\mathrm{jj}}$ Also at Shoolini University, Solan, India. 
${ }^{\mathrm{kk}}$ Also at University of Hyderabad, Hyderabad, India.

${ }^{11}$ Also at University of Visva-Bharati, Santiniketan, India.

${ }^{\mathrm{mm}}$ Also at Indian Institute of Technology (IIT), Mumbai, India.

${ }^{\mathrm{nn}}$ Also at Deutsches Elektronen-Synchrotron, Hamburg, Germany.

${ }^{\circ o}$ Also at Sharif University of Technology, Tehran, Iran.

${ }^{\mathrm{pp}}$ Also at Department of Physics, University of Science and Technology of Mazandaran, Behshahr, Iran.

${ }^{\mathrm{qq}}$ Also at INFN Sezione di Bari, Università di Bari, Politecnico di Bari, Bari, Italy.

${ }^{\text {rr }}$ Also at Italian National Agency for New Technologies, Energy and Sustainable Economic Development, Bologna, Italy.

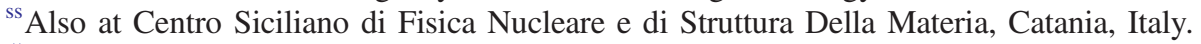

tt Also at Università di Napoli 'Federico II', Naples, Italy.

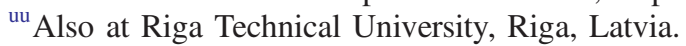

${ }^{\mathrm{vv}}$ Also at Consejo Nacional de Ciencia y Tecnología, Mexico City, Mexico.

${ }^{\text {ww }}$ Also at IRFU, CEA, Université Paris-Saclay, Gif-sur-Yvette, France.

${ }^{\mathrm{xx}}$ Also at Institute for Nuclear Research, Moscow, Russia.

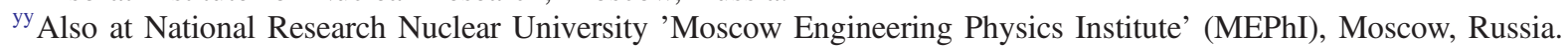

${ }^{\mathrm{zz}}$ Also at Institute of Nuclear Physics of the Uzbekistan Academy of Sciences, Tashkent, Uzbekistan.

${ }^{\text {aaa }}$ Also at St. Petersburg State Polytechnical University, St. Petersburg, Russia.

${ }^{b b b}$ Also at University of Florida, Gainesville, Florida, USA.

${ }^{\mathrm{ccc}}$ Also at Imperial College, London, United Kingdom.

${ }^{\text {ddd }}$ Also at Moscow Institute of Physics and Technology, Moscow, Russia.

${ }^{e e e}$ Also at P.N. Lebedev Physical Institute, Moscow, Russia.

${ }^{\mathrm{fff}}$ Also at California Institute of Technology, Pasadena, California, USA.

${ }^{\text {ggg }}$ Also at Budker Institute of Nuclear Physics, Novosibirsk, Russia.

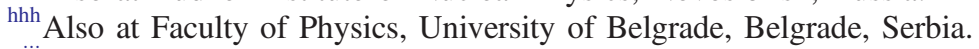

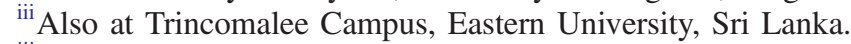

${ }^{j j j}$ Also at INFN Sezione di Pavia, Università di Pavia, Pavia, Italy.

${ }^{k k k}$ Also at National and Kapodistrian University of Athens, Athens, Greece.

${ }^{111}$ Also at Ecole Polytechnique Fédérale Lausanne, Lausanne, Switzerland.

${ }^{\mathrm{mmm}}$ Also at Universität Zürich, Zurich, Switzerland.

${ }^{n n n}$ Also at Stefan Meyer Institute for Subatomic Physics, Vienna, Austria.

${ }^{000}$ Also at Laboratoire d'Annecy-le-Vieux de Physique des Particules, IN2P3-CNRS, Annecy-le-Vieux, France.

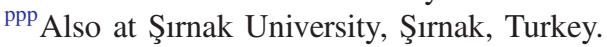

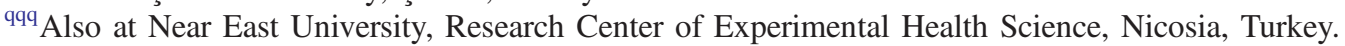

${ }^{\mathrm{rrr}}$ Also at Konya Technical University, Konya, Turkey.

${ }^{\text {sss }}$ Also at Istanbul University_Cerrahpasa, Faculty of Engineering, Istanbul, Turkey.

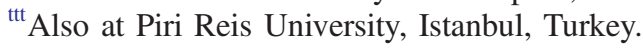

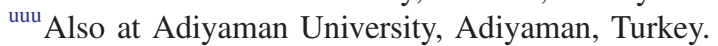

${ }^{\mathrm{vvv}}$ Also at Ozyegin University, Istanbul, Turkey.

${ }^{w w w}$ Also at Izmir Institute of Technology, Izmir, Turkey.

${ }^{\mathrm{xxx}}$ Also at Necmettin Erbakan University, Konya, Turkey.

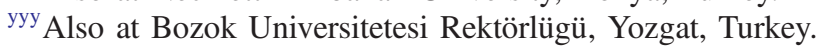

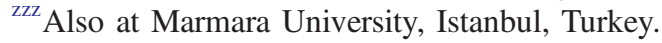

${ }^{\text {aaa }}$ Also at Milli Savunma University, Istnbul, Turkey.

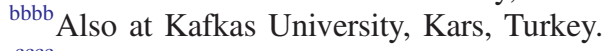

${ }^{c c c c}$ Also at Istanbul Bilgi University, Istanbul, Turkey.

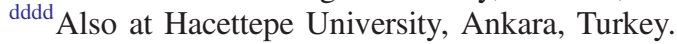

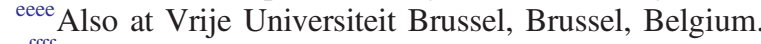

${ }^{\text {ffff }}$ Also at School of Physics and Astronomy, University of Southampton, Southampton, United Kingdom.

${ }^{\text {gggg }}$ Also at IPPP Durham University, Durham, England, United Kingdom.

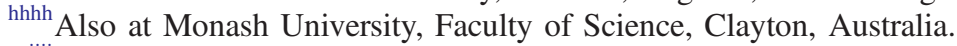

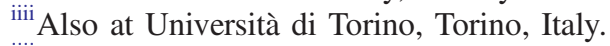

${ }^{j j j j}$ Also at Bethel University, St. Paul, Minneapolis, USA.

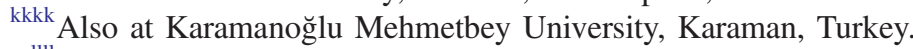

${ }^{1111}$ Also at Ain Shams University, Cairo, Egypt.

${ }^{\mathrm{mmmm}}$ Also at Bingol University, Bingol, Turkey.

${ }^{n n n n}$ Also at Georgian Technical University, Tbilisi, Georgia.

${ }^{\mathrm{oooo}}$ Also at Sinop University, Sinop, Turkey.

${ }^{\text {pppp }}$ Also at Erciyes University, Kayseri, Turkey.

${ }^{\text {qqqq }}$ Also at Texas A\&M University at Qatar, Doha, Qatar.

${ }^{\text {rrrr }}$ Also at Kyungpook National University, Daegu, Korea. 